\title{
Near-Capacity Wireless System Design Principles
}

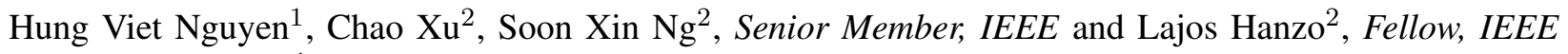 \\ ${ }^{1}$ Posts and Telecommunications Institute of Technology, Vietnam. \\ ${ }^{2}$ School of ECS, University of Southampton, SO17 1BJ, UK. \\ Emails: ${ }^{1}$ hungnv_vt1@ptit.edu.vn; ${ }^{2}\{\mathrm{cx} 1 \mathrm{~g} 08, \mathrm{sxn}, \mathrm{lh}\} @$ ecs.soton.ac.uk;
}

\begin{abstract}
In this tutorial, the design procedure of nearcapacity channel code design invoking non-binary EXtrinsic Information Transfer (EXIT) charts is illustrated by using design examples of Irregular Concatenated Coding Arrangements (ICCAs) relying on Irregular Convolutional Codes (IrCCs). More specifically, in order to benchmark the near-capacity design examples, both the capacity and the Outage Probability (OP) of the Continuous-input Continuous-output Memoryless Channel (CCMC), of the Discrete-input Continuous-output Memoryless Channel (DCMC) as well as of the Differential Discreteinput Continuous-output Memoryless Channel (D-DCMC) are characterised. Furthermore, the EXIT-chart aided near-capacity design principles are illustrated by detailing the design process of three characteristic prototype schemes, which represent the family of coherent and non-coherent detection based systems as well as Multi-Input Multi-Output (MIMO) systems, respectively. Moreover, the beneficial application of the EXIT-chart based design principle is also demonstrated in the context of cooperative systems using a specific distributed MIMO prototype scheme.
\end{abstract}

\section{INTRODUCTION}

The ultimate aim of designing a wireless communication system is to provide reliable high data rate links. Shannon's pioneering work published in 1948 [1] predicted that arbitrarily reliable communications are achievable with the aid of channel coding, provided that the required amount of redundant information is imposed on the transmitted messages. A breakthrough in channel coding history was the invention of turbo codes [2] in 1993, which may be employed for facilitating near-capacity operation. The original turbo code [3] design was based on a 'composite codec' constituted by two parallel concatenated codecs. Originally, the concatenated coding philosophy was proposed by Forney in 1966 [4], but until the 1990s it was only employed in demanding applications, such as deep-space communications owing to its high complexity. However, following the revolution turbo concept, since the 1990s it has found favour in numerous concatenated coding schemes [5]-[8].

Advances in channel coding made it feasible to gradually approach Shannon's capacity limit in Single Input Single Out (SISO) systems equipped with a single antenna [9]. However, these capacity limits can be further extended with the aid of multiple antennas in Multiple Input Multiple Output (MIMO) systems, which are capable of providing a linearly increasing capacity as a function of the transmit power, provided that the extra power is assigned to additional antennas [10]. Space

The financial support of the Vietnamese International Education Development (VIED) fund and that of the European Union's Seventh Framework Programme (FP7/2007-2013) under the auspices of the CONCERTO project (grant agreement no 288502) is gratefully acknowledged.
Time Trellis Codes (STTCs) [11] and Space Time Block Codes (STBCs) [12] constitute a family of joint coding and transmit-receive diversity aided MIMO systems designed for communicating over fading channels [13]. However, it is impractical to allocate multiple antennas to a shirt-pocketsized mobile unit. In order to circumvent this space-limitation, the concepts of cooperative communication have recently attracted substantial research efforts [14]-[18], since they are capable of creating a Virtual MIMO (VMIMO) system from several single-antenna aided relays, which cooperatively share their antennas. The important milestones of specific advances related to the subject of this tutorial are listed in Tab. I.

EXtrinsic Information Transfer (EXIT) chart introduced by Stephan ten Brink [32] is a very useful tool used for characterising an iterative process of information transfer between two decoders of turbo codes, which is not easy to analyse and to describe. Based on specific technique of iterative information exchange among the component codes, a concatenated coding scheme can be categorised into three major types [33] as seen in Fig. 1, namely:

- Parallel Concatenated Convolutional (PCC) codes comprise two or more parallel component codes iteratively exchanging mutual information (MI) with the aid of an interleaver. The component codes may be Recursive Systematic Convolutional (RSC) codes [3], Unity Rate Codes (URC) [34] or Trellis Coded Modulation (TCM) codes [35].

- Self-concatenated Convolutional (ScC) codes rely on a single component encoder combined with a turbo interleaver at the transmitter side. The decoder relies on using a single decoder twice [33], [36].

- Serial Concatenated Convolutional (SCC) codes are typically constructed by concatenating $M$ component codes in order to constitute a more powerful amalgamated code, where $M$ component encoders are separated by $M-1$ interleavers [8], [37].

As classic Turbo Codes (TCs) [2] consist of two or more parallel constituent codes representing PCC codes, while a tutorial of $\mathrm{ScC}$ codes has been presented in [33], let us pay attention on the SCC codes. The SCC codes have recently provoked a number of research work focusing on near-capacity EXIT chart aided coding designs [38]-[55], which may be categorised into several sub-areas, as seen in Fig. 1, namely SISO schemes based on coherent detection [40], [41], [43], Noncoherent detection based SISO schemes [50], [51], MIMO schemes [39], [44], [46]-[48], [53], [54], VMIMO schemes 


\begin{tabular}{|c|c|c|}
\hline Year & Author & Milestone \\
\hline 1948 & Shannon [1] & Introduced Shannon's capacity theorem. \\
\hline 1955 & Elias [19] & Introduced convolutional codes. \\
\hline 1966 & Forney [4] & Introduced concatenated codes. \\
\hline 1971 & van der Meulen [20] & Introduced a simple relay channel constituted by a source, a destination and a relay. \\
\hline 1972 & Bahl et al. [21] & Invented the Maximum A-Posteriori (MAP) algorithm. \\
\hline 1974 & Bahl et al. [22] & Proposed the symbol based MAP algorithm. \\
\hline 1979 & Cover and El Gamal [23] & Presented capacity analysis of the full duplex relay channel. \\
\hline 1993 & Berrou et al. [2] & Discovered turbo codes. \\
\hline 1995 & Douillard et al. [24] & Proposed turbo equalisation \\
\hline \multirow[t]{2}{*}{1997} & Tarokh et al. [25] & Introduced Space Time Trellis Coding (STTC). \\
\hline & Benedetto et al. [26] & $\begin{array}{l}\text { Investigated iterative decoding of an outer convolutional decoder and } \\
\text { an inner Trellis-Coded Modulation (TCM) decoder. }\end{array}$ \\
\hline \multirow[t]{2}{*}{1998} & Alamouti et al. [27] & Proposed Space Time Block Coding (STBC). \\
\hline & Benedetto et al. [8], [28] & $\begin{array}{l}\text { Derived design guidelines for serially concatenated codes with the aid of the union-bound } \\
\text { technique [8], and also extended the turbo principle to multiple serially concatenated codes [28] }\end{array}$ \\
\hline 1999 & ten Brink [29] & Proposed EXIT charts for analysing the convergence behaviour of iterative decoding and demapping. \\
\hline 2002 & Tücher and Hagenauer [30] & $\begin{array}{l}\text { Proposed irregular convolutional codes as well as a simplified method for } \\
\text { computing mutual information in EXIT chart analysis }\end{array}$ \\
\hline 2004 & Ashikhmin et al. [31] & $\begin{array}{l}\text { Presented a universal model for conducting EXIT chart analysis as well as various properties } \\
\text { of EXIT functions, which form the theoretical foundation of EXIT-curve matching techniques }\end{array}$ \\
\hline
\end{tabular}

TABLE I

Milestones in CHANNEL CODING OF InTERESTS FROM Single INPUT Single OUTPUT (SISO) To MIMO.

[42], [45], [49], [51] and joint source-channel coding schemes [38], [55]. As a result, the authors of [56] have published a tutorial focusing on merely in coherent SISO schemes, while in another tutorial [57] the authors have covered the specific subject-area of joint source-channel coding. Hence, as explicitly portrayed in Fig. 1, this treatise aims for providing a broad tutorial on the application of EXIT charts for nearcapacity IrCC aided designs. Specifically, we illustrate the associated principles with the aid of design examples in the general context of taking into account both coherent and noncoherent detection, MIMO scenarios as well as cooperative communications.

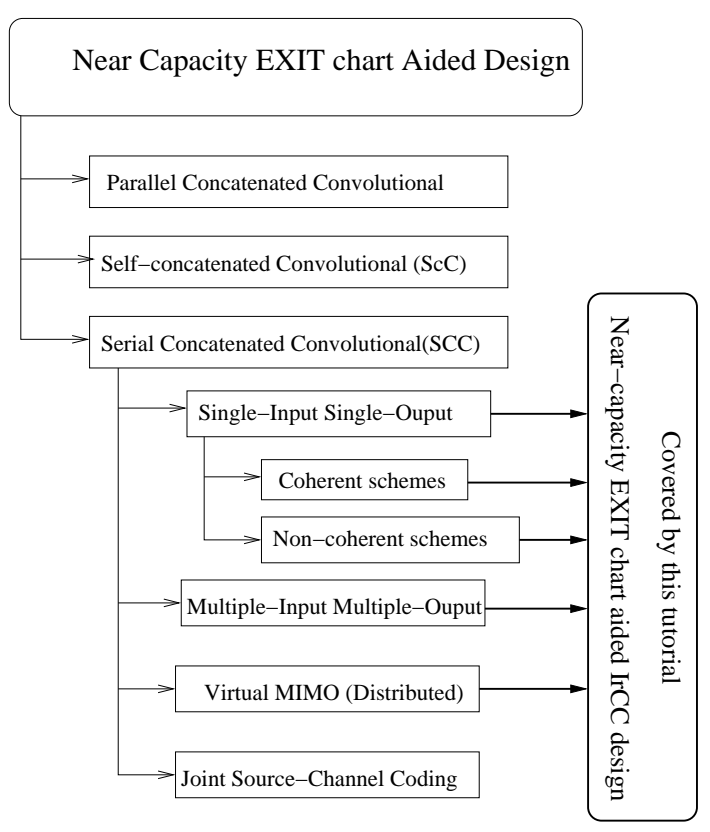

Fig. 1. Taxonomy of near-capacity EXIT aided designs in the literature.

More specifically, in this tutorial we will summarise the techniques of near-capacity code-design with the assistance of four prototype systems designed upon using the EXIT chart base tool. Accordingly, we will illustrate the basic design principles with the aid of three stage concatenated coding schemes employing an irregular outer code, for example IrCCs. The intermediate code employed for constructing the three-stage concatenated coding schemes is constituted by URC [58]. It was shown in [59], [60] that a recursive URC should be employed as an intermediate code in order to improve the attainable decoding convergence. More specifically, we will show that a URC can be used as a precoder for creating an inner code component having an Infinite Impulse Response (IIR) in order to reach the $(1,1)$ point of perfect decoding convergence in the EXIT chart and hence to achieve an infinitesimally low Bit Error Ratio (BER) [61]. The inner most stage can be a coherent/noncoherent base detector itself for the coherent/noncoherent schemes or STTCs for the MIMO and VMIMO schemes.

To elaborate a little further, in order to benchmark the near-capacity designs considered, we will detail the specifics of calculating both the capacity and the outage capacity, which may be determined by employing EXIT charts. These capacities represent the best possible performance of corresponding coding schemes in both small-scale and largescale fading environments. The best possible performance may be achieved, when an ideal/perfect capacity-achieving coding scheme is employed. Accordingly, the EXIT chart based design principle will be exemplified for both coherent and non-coherent detection based SCC schemes. Additional discussions on the effect of realistic channel estimation errors will also be presented in order to highlight both the pros and cons of the coherent and non-coherent modulation based schemes. Our discussions are continued by highlighted points on the benefit of employing sub-frame based transmissions in slow-fading environments, where the outage capacity should be used for benchmarking the near-capacity coding schemes considered. Moreover, the paper continues by describing the application of the design principles in the context of coop- 
erative communications, where distributed SCC schemes are employed, as seen in Fig. 1.

The rest of the paper is characterised by the stylised illustration of Fig. 2, where all the major subjects presented in the papers are visualised along with the relationships between them. More specifically, our paper is focused on the subject of near-capacity EXIT-chart aided system design. Explicitly, we present the basic design principles in Section V-A, which are then exemplified in Section V-B, Section V-C and Section VI with the aid of prototype designs. The design process is relied on the employment of the EXIT chart design tool, which is briefly characterised in Section II-A and Section V-A. Finally, we provide our generalised design guidelines in Section VII-B.

Observing Fig. 2 from a different perspective, the nearcapacity design of wireless systems considered in the context of the tutorial is characterised by three groups of parameters, namely by the coding components, transmission schemes and the associated conflicting metrics. Accordingly, we provide the relevant background on the coding components used, namely on IrCCs in Section II-B and on STTCs in Section II-C, while the transmission regimes covering both the class of coherent and non-coherent systems are detailed in Section V-B and Section $\mathrm{V}-\mathrm{C}$, where the related issues of the channel estimation are also covered in Section V-E. Moreover, additional discussions are also extended to different channel fading types, namely to both fast fading and slow fading, in order to highlight the benefit of employing sub-frame based transmission regimes in Section V-D. As regards to the transmission schemes, the tutorial covers the concepts of SISO schemes in Section V-B and Section V-C, MIMO schemes in Section VI-C, whilst those of VMIMO schemes in Section VI-B and Section VI-D. The metrics used for those schemes, namely the outage probability and outage capacity of the schemes, are presented in Section III and Section IV, respectively.

\section{PReliminaries}

In this section, we will provide background pertaining to the EXIT charts, IrCC, STTC and non-coherent detection, in order to facilitate the presentation of our formal tutorial paper on near-capacity EXIT chart aided IrCC design in the general context taking into account of coherent/non-coherent detection, MIMO scenarios and cooperative communications.

\section{A. Extrinsic Information Transfer Charts}

As more and more complicated concatenated codes are constructed for the sake of pursuing a near-capacity performance, one of the major design challenge is to predict and compare their $E_{b} / N_{0}$ convergence thresholds in order to choose the most appropriate channel coding and modulation parameters. Motivated by this problem, researchers have focused their attention on characterizing the convergence behavior of turbo codes [32], [62]-[66], which rely on the 'turbo principle'. In its basic form, the turbo principle is employed in the context of two concatenated coding components, namely an inner and an outer components, albeit there are also powerful threecomponent or multiple-component [34], [67] schemes. The salient benefit of the turbo principle is that the inner and outer components exchange soft values. Accordingly, the extrinsic part of the soft-output of one decoder can be passed to the other decoder to act as its a priori input. This decoding process constitutes an iterative information transfer between the two turbo decoders, which can be visualised with the aid of EXIT charts [68].

Several measures have been proposed for characterising the iterative process of turbo codes, which were listed and compared in [69], amongst which, the soft bit transfer (SOBIT) chart and the EXIT chart appealingly visualise the behaviour of the iterative decoding process [69]. Moreover, according to the comparisons in [68], the EXIT chart was considered to be physically more meaningful than the SOBIT chart due to its compelling information theoretical interpretation relying on fractions of a bit.

Generally, an EXIT chart of a serially concatenated scheme visualises the exchange of four MI terms, namely the average $a$ priori $\mathrm{MI}$ of the inner component $I_{A}^{i n}$, the average a priori MI of the outer component $I_{A}^{\text {out }}$, the average extrinsic MI of the inner component $I_{E}^{i n}$ and the average extrinsic MI of the outer component $I_{E}^{\text {out }}$. More specifically, the $I_{A}^{i n}$ and $I_{E}^{i n}$ constitute the EXIT curve of the inner component, while their outercomponent based counterpart is formed by $I_{A}^{\text {out }}$ and $I_{E}^{\text {out }}$. For convenience, the MI transfer characteristics of both the inner and outer components are plotted in a single graph, where the $\mathrm{x}$ and $\mathrm{y}$ axes of the outer decoder are swapped for each new information exchange step. The resultant EXIT chart quantifies the improvement in mutual information as the iterations proceed, which results in a stair-case-shaped decoding trajectory.

In the rest of this section, we provide insights into the stateof-the-art EXIT chart [32], [65], [66], which can be used for effectively analysing the flow of extrinsic information between turbo coding components so that decoding convergence may be accurately predicted. Accordingly, both the rudimentary theoretical principles as well as the practical applications of EXIT charts will be elaborated on.

1) Mutual Information and Transfer Characteristics: Assume that a data bit $b \in\{1,0\}$ at the transmitter is equiprobable. In order to investigate the flow of MI at a component block at the receiver, the transfer characteristics of the component may be formulated as [32], [70]:

$$
I_{E}=T\left(I_{A}\right),
$$

where the a priori information $I_{A}$ and the extrinsic information $I_{E}$ at the input and at the output of the transfer function $T$ are given by $I_{A}=I\left(b ; \Lambda_{a}\right)$ and $I_{E}=I\left(b ; \Lambda_{e}\right)$, while $\Lambda_{a}$ and $\Lambda_{e}$ are random variables modelled for $L_{a}$ and $L_{e}$, respectively. Note that the notation $I(X ; Y)$ represents the MI between random variables $X$ and $Y$ [71], while $L_{a}$ and $L_{e}$ are the corresponding Loglikelihood Ratio (LLR) representing a soft-value input and a soft-value output of the component, respectively. For more details about LLR relating to turbo principle, please allow us to refer readers to the tutorial presented in [68].

The first step of obtaining $I_{E}$ with given $I_{A}$ is to generate a group of a priori LLRs $L_{a}$ from values of $I_{A}$. Then, $I_{E}=$ $I\left(b ; \Lambda_{e}\right)$ may be evaluated accordingly, where the extrinsic 


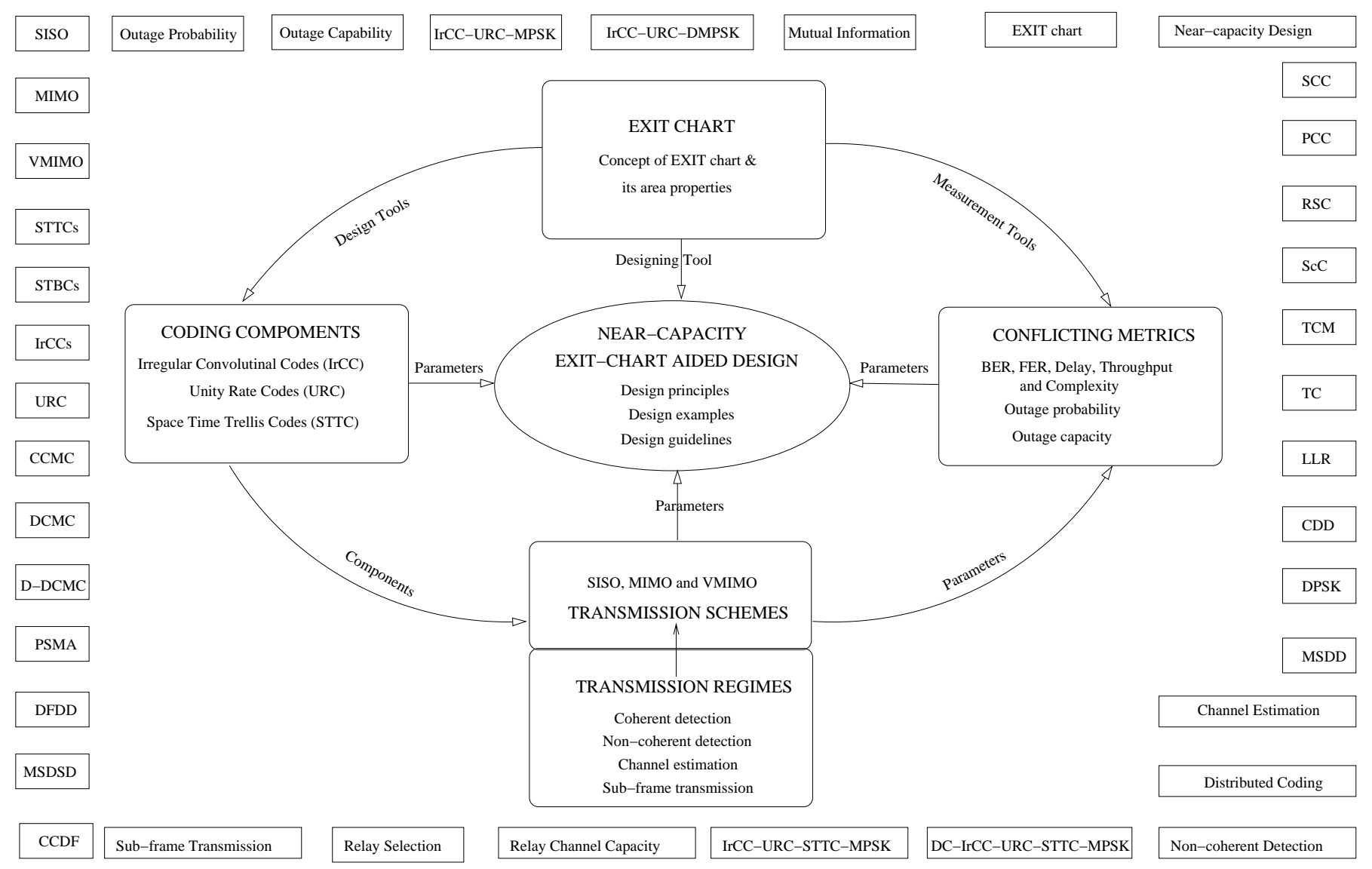

Fig. 2. Outline and rationale of the paper.

LLRs $L_{e}$ are obtained by feeding $L_{a}$ to the component under study.

Assume that long interleavers are employed between concatenated components so that the LLRs exchanged by them are uncorrelated and Gaussian-distributed. Therefore, the Gaussian-distributed LLRs of $L_{a}$ may be generated [32]. Accordingly, the formula of $I_{A}$ may be simplified by an inputoutput relationship characterised as a function $J(\cdot)$ as:

$$
I_{A}=J\left(\sigma_{A}\right)
$$

where $\sigma_{A}^{2}$ obeying $\sigma_{A} \geq 0$ is a variance of a Gaussian random variable constituting the value of $L_{a}$, while $0 \leq I_{A} \leq$ 1 is satisfied. Furthermore, function $J(\cdot)$ of Eq. (2) has the following properties:

$$
\lim _{\sigma_{A} \rightarrow 0} J\left(\sigma_{A}\right)=0, \quad \lim _{\sigma_{A} \rightarrow \infty} J\left(\sigma_{A}\right)=1 .
$$

It is observed that the output $I_{A}$ of function $J(\cdot)$ increases monotonically with respect to input $\sigma_{A}$. Although the function $J(\cdot)$ and its inverse function $J^{-1}(\cdot)$ cannot be expressed in closed form [32], we refer to [72] for the approximate form of the functions $J(\cdot)$ and $J^{-1}(\cdot)$.

As a result, we can summarise the procedures of evaluating the transfer characteristics of Eq. (1) as:

(1) For given a specific a priori mutual information $I_{A}$, a group of a priori LLRs $L_{a}$ may be generated [32], where the standard deviation $\sigma_{A}$ of the Gaussian distribution is given by the inverse function of $J(\cdot)$ [72], i.e. we have $\sigma_{A}=J^{-1}\left(I_{A}\right)$.

(2) Feed the generated soft-value inputs $L_{a}$ to the tested component in the concatenated code, and then a group of extrinsic LLR outputs $L_{e}$ may be obtained.

(3) Finally, the extrinsic MI output of Eq. (1) may be computed upon evaluating the histograms of $L_{e}$ [73] with respect to the source data bit being $b=1$ and $b=0$.

2) Area Property: EXIT charts have been introduced as an effective tool conceived for analysing the convergence properties of iterative decoding aided concatenated coding schemes [74]. By exploiting two basic properties:

- EXIT charts may be used for visually characterising the flow of soft-information between component codes of concatenated coding schemes. Given the EXIT chart of a concatenated coding scheme, the Signal to Noise Ratio $(S N R)$, at which an infinitesimally low BER of the scheme is achieved, may be predicted by exploiting that once the Monte-Carlo simulation based bit-by-bit decoding trajectory reaches the $(1,1)$ point of the EXIT chart, perfect decoding convergence has been attained. Hence, no further decoding iterations are required. In other words, the turbo-cliff SNR of the concatenated coding scheme may be found by merely looking at the EXIT chart. Moreover, upon counting the number of stair-case-shaped steps in the decoding trajectory curves, 
the appropriate number of iterations between two coding components may be determined.

- The area under the EXIT curve of an inner decoder component is approximately equal to the attainable channel capacity, provided that the channel's input symbols are equiprobable [31], [75]. This property [31], [75] may be exploited for determining the achievable rates of Forward Error Control (FEC) schemes relying on iterative multi-stage coding. Then, the achievable capacity may be used for selecting a specific coding scheme from the available set and may also be used for optimising coding arrangements relying on numerous parameters by using the achievable capacity as a criterion for comparing all legitimate sets of parameters used for specifying the coding arrangement. As an advantage, a near-capacity code design can be found by employing EXIT charts without performing time-consuming Monte-Carlo simulations. EXIT charts can also be used for finding powerful codes exhibiting guaranteed convergence for a given channel [76]. Specifically, near-capacity codes have been successfully designed by applying an EXIT-chart-based technique, for example in [30], [61], [77].

\section{B. Irregular Convolutional Codes}

The irregular design of channel codes has received significant scientific attention from a wide range of researchers for the sake of characterising the irregularity of low density parity check codes (LDPC) [63], [78]-[80], of accumulate codes [80]-[84], of variable length codes [38], [40] as well as of convolutional codes [39], [44], [45], [51]. In the frame work of this tutorial, we focus our attention on the family of irregular convolutional codes, which constitute the juxtapositioned counterpart of classic PCCs, ScSs and SCCs seen Fig. 1. Since the literature of PCC and ScC structures has been covered in [33], [56], the goal of this tutorial is to present the suit of irregular convolutional codes in the context of both coherent/non-coherent detection, MIMO scenarios and cooperative communication. By contrast, the subject of joint source-channel coding has been presented in [57], as indicated in Fig. 1.

In the framework of this paper, let us commence by considering a classic two-stage SCC, where the two component codes may be deemed as inner code and outer code. A variety of near-capacity systems [32], [46], [85]-[87] aim to match the EXIT curves of inner and outer codes, so that an open tunnel may be encountered at the lowest possible $E_{b} / N_{0}$. For the extreme situation of having an infinitesimally small open tunnel, we have a perfect match of the inner and outer codes, where the maximum achievable rate is achieved. Therefore, a near-capacity system's design may aim for achieving an infinitesimally low BER at an $S N R$, so that no transmit power should be wasted.

In order to achieve this goal, on one hand, numerous researchers have focused their attentions on how to design optimised modulation schemes so that their EXIT curves may fit closer to the outer channel code's EXIT curve. This topic is widely known as mapping optimization for Bit-Interleaved
Coded Modulation with Iterative Decoding (BICM-ID) [86], [88]-[92]. On the other hand, as the family of modulation schemes keeps growing especially for MIMO applications, it becomes more feasible to adjust channel coding's transfer characteristics as illustrated in [46], [87], [93], where IrCCs is a popular application of the aforementioned near capacity design.

Tüchler and Hagenauer [30], [87] proposed the employment of IrCCs for the design of near-capacity serial concatenated schemes, which are constituted by a family of convolutional codes having different rates. These IrCCs were specifically designed with the aid of EXIT charts for improving the convergence behaviour of iteratively decoded systems. Briefly, these schemes were then further developed for a broad class of turbo-transceivers in [85]. Each subcode $\phi$ encodes an appropriately selected fraction $\alpha_{\phi}$ of the input bit stream, where $\phi=[1,2, \ldots, \Phi]$. More explicitly, the appropriate fractions may be selected with the aid of EXIT-chart analysis in order to shape the inverted EXIT curve of the composite IrCC for ensuring that it matches the EXIT curve of the socalled inner decoder constituted by the demodulator/detector. In this manner, an open EXIT-chart tunnel can be created at low SNR values, which implies approaching the channel's capacity bound, as detailed in [31]. In our example, we choose either $\Phi=17$ or $\Phi=36$ for illustrating our design principles. Accordingly, if the $\phi^{\text {th }}$ subcode having a code rate $\beta_{\phi}$ encodes the fraction $\alpha_{\phi}$ of the input bit stream, where the values of $\beta_{\phi}$ are listed in Tab. II, the values of the weighting coefficient $\alpha_{\phi}$ and subcode rate $\beta_{\phi}$ always have to satisfy the constraint of:

$$
R_{c}=\sum_{\phi=1}^{\Phi} \alpha_{\phi} \beta_{\phi},
$$

where $R_{c}$ is the coding rate (aggregate coding rate) of the IrCC. As a result, the EXIT curve of each IrCC subcode

\begin{tabular}{|l|l|l|l|}
\hline Subcode & Code Rate $\beta_{\phi}$ & Subcode & Code Rate $\beta_{\phi}$ \\
\hline 1 & $\beta_{1}=0.10$ & 18 & $\beta_{18}=0.10$ \\
\hline 2 & $\beta_{2}=0.15$ & 19 & $\beta_{19}=0.15$ \\
\hline 3 & $\beta_{3}=0.20$ & 20 & $\beta_{20}=0.20$ \\
\hline 4 & $\beta_{4}=0.25$ & 21 & $\beta_{21}=0.25$ \\
\hline 5 & $\beta_{5}=0.30$ & 22 & $\beta_{22}=0.30$ \\
\hline 6 & $\beta_{6}=0.35$ & 23 & $\beta_{23}=0.35$ \\
\hline 7 & $\beta_{7}=0.40$ & 24 & $\beta_{24}=0.40$ \\
\hline 8 & $\beta_{8}=0.45$ & 25 & $\beta_{25}=0.45$ \\
\hline 9 & $\beta_{9}=0.50$ & 26 & $\beta_{26}=0.50$ \\
\hline 10 & $\beta_{10}=0.55$ & 27 & $\beta_{27}=0.45$ \\
\hline 11 & $\beta_{11}=0.60$ & 28 & $\beta_{28}=0.50$ \\
\hline 12 & $\beta_{12}=0.65$ & 29 & $\beta_{29}=0.55$ \\
\hline 13 & $\beta_{13}=0.70$ & 30 & $\beta_{30}=0.60$ \\
\hline 14 & $\beta_{14}=0.75$ & 31 & $\beta_{31}=0.65$ \\
\hline 15 & $\beta_{15}=0.80$ & 32 & $\beta_{32}=0.70$ \\
\hline 16 & $\beta_{16}=0.85$ & 33 & $\beta_{33}=0.75$ \\
\hline 17 & $\beta_{17}=0.90$ & 34 & $\beta_{34}=0.80$ \\
\hline & & 35 & $\beta_{35}=0.85$ \\
\hline & & 36 & $\beta_{36}=0.90$ \\
\hline
\end{tabular}

TABLE II

LIST OF THE SUBCODE RATES FOR THE IRCC CODE HAVING $\Phi=17$ OR $\Phi=36$ SUBCODES

is provided Fig. 3, where superposition of EXIT curves of 
the subcodes selected from $\Phi$ available subcodes are plotted. It should be noted that each EXIT curve is generated by employing the algorithm detailed in Section II-A1 and that the IrCC is equivalent to the subcode of interest if the corresponding fraction of the subcode is set to one.
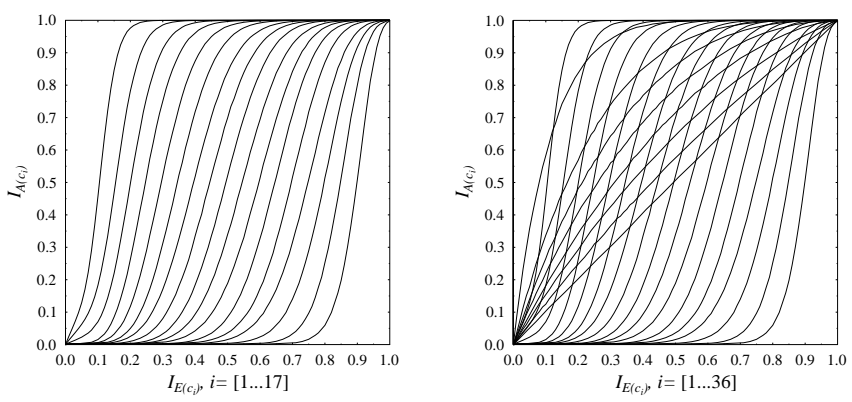

Fig. 3. EXIT curves of $\Phi$ IrCC subcodes, where $\Phi=17$ or $\Phi=36$.

\section{Space Time Trellis Code}

As mentioned in Section I, STTCs [11] and STBCs [12] constitute a family of joint coding and transmit-receive diversity aided MIMO systems designed for communicating over fading channels [13]. We note, however that in contrast to STBCs, STTCs are also capable of attaining a coding gain in addition to their spatial diversity gain, while again, the STBCs of [76] can only achieve a spatial diversity gain [85] but no coding gain. As a result, we opted for using the STTCs in our subsequent prototype design examples for demonstrating the EXIT chart based design principles detailed in Section V-A.

STTCs [11], [13], [94], [95] were proposed by Tarokh et al., which incorporate jointly designed channel coding, modulation, transmit diversity and optional receiver diversity. The performance criteria for designing STTCs were listed in [11] with the assumption that the channel is fading slowly and that the fading is non-dispersive. It was illustrated in [11] that the systems performance is determined by matrices constructed from pairs of distinct code sequences. The diversity gain and coding gain of the codes are determined by the minimum rank and the minimum determinant [11], respectively. The results were then also extended to fast-fading channels [96]. The STTCs proposed in [11] strike the best trade-off among the data rate, diversity gain and trellis complexity. The effect of multiple propagation paths on the performance of STTCs were investigated in [13] for transmission over slowly varying Rayleigh fading channels. It was shown in [13] that the presence of multiple paths does not decrease the diversity order guaranteed by the design criteria used for constructing the STTCs. The results provided in [13] were then also extended to rapidly-fading dispersive and non-dispersive channels.

Having presented a rudimentary introduction to STTCs, we refer the motivated readers to [11] for the details of encoding and decoding processes of the STTCs. A specific example employed for our subsequent illustrations is that of the 4state, 4-level Phase Shift Keying (4PSK) STTC using $N_{t}=2$ transmit antennas. More sophisticated STTCs were designed for 4PSK and 8PSK [11] by increasing the number $S$ of trellis states to $S=\{8,32\}$.

\section{Non-coherent detection}

The early development of Differential Phase Shift Keying (DPSK) may be found in [97], [98], where the data carrying symbol $x_{n-1}$ is mapped on to the change of the consecutive transmitted symbols as $s_{n}=x_{n-1} s_{n-1}$. As a result, in AWGN channels where both a Gaussian noise $v_{n}$ and a constant frequency offset phase $\exp (j \theta)$ contaminate the received signal as $y_{n}=s_{n} \exp (j \theta)+v_{n}$, the simple non-coherent detection operation of $z_{n-1}=y_{n} y_{n-1}^{*}$ may eliminate the need for estimating the unknown phase for recovering $x_{n-1}$. This low-complexity non-coherent detection, which detects a single symbol based on two observations may be termed as Conventional Differential Detection (CDD). However, the CDD aided DPSK suffers from a $3 \mathrm{~dB}$ performance penalty compared to its idealistic coherent PSK counterpart assuming perfect estimation of the phase offset [99]. Moreover, an irreducible error floor occurs for DPSK [100], when the CDD is employed in rapidly fluctuating high-Doppler fading channels.

The pragmatic approach of Multiple Symbol Differential Detection (MSDD) conceived for improving non-coherent detection was proposed in [101], [102] for DPSK reception in AWGN channels. In the approach, the symbols in an adjustable detection window relying on $N_{w}$ received samples are jointly detected. The MSDD designed for DPSK was then further extended for DPSK operating in fading channels [103], [104]. In the extension, upon increasing the detection window length $N_{w}$, MSDD is not only capable of mitigating the $3 \mathrm{~dB}$ performance gap between non-coherent detection and coherent detection in AWGN channels, but it is also capable of reducing the error floor by exploiting the correlation of the fading envelope. However, the MSDD complexity may increase exponentially with the window length $N_{w}$.

In order to reduce the MSDD complexity, an efficient algorithm was conceived for MSDD in AWGN channels [105], where a low detection complexity order of $O\left(\log N_{w}\right)$ was imposed by detecting a single symbol upon observing the phase changes over the $N_{w}$ samples. This method may also be applied for improving the performance of the CDD aided DPSK in block fading channels. It is due to the fact that accurate channel estimation may be implemented at a low cost in slowly fluctuating fading channels. For example, Pilot Symbol Assisted Modulation (PSAM) was proposed in [106], where the known pilot samples may be transmitted periodically. Accordingly, the receiver may recover the complexvalued fading factors by estimating the channel with the aid of pilot samples. However, when the fading fluctuates rapidly, the PSAM aided coherent scheme has to dedicate more transmission power to the pilot symbols instead of the datacarrying symbols. Despite of this, the accuracy of channel estimation also degrades in comparison to the case of block fading. Therefore, the implementation of the MSDD at an affordable complexity is desirable in the context of rapidly fading channels.

Accordingly, the Decision-Feedback Differential Detection (DFDD) was proposed for DPSK operating in AWGN channels [107], [108] and was further detailed in [109]. The DFDD 
makes a decision regarding a single symbol based on $\left(N_{w}-1\right)$ previous decisions. As a result, the DFDD has a low detection complexity order of $O(M)$, when detecting a single symbol. The DFDD designed for DPSK was then further extended to fading channels [110], [111] in the context of coded DPSK. The DFDD is considered to be equivalent to the MSDD associated with decision feedback, but the DFDD fails to approach the optimum MSDD detection capability. In order to mitigate this problem, Multiple Symbol Differential Sphere Detection (MSDSD) was proposed in [112] for uncoded DPSK and in [113] for coded DPSK, where a sphere decoder was invoked for implementing MSDD. The MSDSD complexity is lower bounded by $O(M)$, but the algorithm exhibits its highest complexity in the low SNR region [114], [115].

\section{OUtAGE PROBABILITY AND CHANNEL CAPACITY}

The outage probability (OP) can be used for characterising the Quality of Service (QoS) of diverse systems. For example, the OP of the idealised transmission link operating exactly at the CCMC channel's capacity may be used as a benchmarker relying on Gaussian distributed signals, as detailed in Section III-A. By contrast, the OP of a specific digital modulation scheme corresponding to the DCMC of Section III-B and to the D-DCMC of Section III-C may be used as tighter and more realistic bounds.

Let us commence by considering the model of a single transmission link associated with the transmitted and received signals of $x$ and $y$, respectively. The received signal can be represented as

$$
y=h x+n,
$$

where $h=h_{s} h_{f}$ is the complex-valued fading coefficient that comprises two components, namely a block fading coefficient (slow fading, large-scale shadow fading or quasi-static fading) $h_{s}$, which is constant for all symbols within a frame and a fast fading (small-scale fading) coefficient $h_{f}$, which fluctuates on a symbol-by-symbol basis. Finally, $n$ is the AWGN process having a variance of $N_{0} / 2$ per dimension.

We refer to $C$ as the maximum achievable transmission rate of reliable communication supported by the channel characterised by (5). Let us assume that the transmitter encodes data at a rate of $R$ bits $/ \mathrm{s} / \mathrm{Hz}$. If the channel realisation $h$ has a capacity of $\left.C\right|_{h}<R$, which is lower than required, the system is declared to be in outage, where the OP is given by:

$$
P_{e}(R)=\operatorname{Pr}\left\{\left.C\right|_{h}<R\right\},
$$

with $\left.C\right|_{h}$ being the capacity, i.e. the maximum achievable rate of the channel, when $h$ is known.

\section{A. Continuous-Input Continuous-Output Memoryless Channel}

We may be able to calculate the OP of the CCMC channel by applying the principle represented by Eq. (6) for the CCMC model. Accordingly, the OP of the interleaved channel associated with a transmission frame length of $N$ symbols spanning from $N \longrightarrow \infty$ can be formulated as [116], [117]

$$
P_{e}^{C C M C}(R)=\operatorname{Pr}\left\{\left|h_{s}\right|^{2} \mathrm{E}\left[\left|h_{f}\right|^{2}\right]<\frac{2^{R}-1}{S N R}\right\},
$$

where it was exploited that the block fading coefficient $h_{s}$ remains constant for all $N$ symbols of a transmitted frame and faded independently for the next transmit frame. This can be directly inferred from Eq. (7) for the OP corresponding to the scenario in which the signals experience small-scale fading as well as for the case of signals that do suffer from block fading, but no small-scale fading. Note that in the block fading scenario, the OP is equivalent to the Frame Error Ratio (FER) of the corresponding transmission link.

\section{B. Discrete-Input Continuous-Output Memoryless Channel}

For a specific modulation order, for example Binary PhaseShift Keying (BPSK), Quadrature Phase-Shift Keying (QPSK), it is more practical to employ the DCMC capacity and the associated OP for benchmarking an associated near-capacity channel coded modulation scheme. Thus, in this section we first review the capacity calculation of a DCMC channel, before detailing the steps to be followed for determining the corresponding outage capacity.

More specifically, the reference of [118] details formulae for calculating the corresponding channel capacity of a transmission link operating over the DCMC channel, over which a specific modulation scheme is advocated. The modulation scheme is characterised by $L=2^{\eta}$ known as the number of modulation levels, while $\eta$ is the number of modulated bits. For example, Fig. 4 presents capacity curves of the DCMC channel influenced by uncorrelated Rayleigh (smallscale) fading, when employing BPSK, QPSK, 8 level Phase Shift Keying (8PSK) and 16 level Phase Shift Keying (16PSK) schemes. These channel capacities of Fig. 4 are evaluated by employing the classic Monte Carlo simulation method for averaging the expectation terms, which can be modified for covering the scenario of MIMO systems, as detailed in [118].

In order to define the OP for the DCMC scenario, let us denote the receiver's faded signal to noise power ratio as $S N R_{r}=E\left[|h|^{2} S N R\right]$. At a given throughput $R$, one can readily identify the corresponding signal to noise power ratio $\left.S N R_{r}\right|_{R}$ that is defined as the SNR value associated with the capacity $C_{(\eta)}^{D C M C}=R$ on the $C_{(\eta)}^{D C M C}$-versus- $S N R$ capacity curve plotted in Fig. 4. Then, similar to the philosophy applied for the CCMC scenario reflected by Eq. (7), the OP of the interleaved DCMC model is equivalent to the probability of the event that we have $|h|^{2} S N R<\left.S N R_{r}\right|_{R}$, which is expressed as

$$
P_{e}^{D C M C}(R, \eta)=\operatorname{Pr}\left\{\left|h_{s}\right|^{2} \mathrm{E}\left[\left|h_{f}\right|^{2}\right]<\frac{\left.S N R_{r}\right|_{R}}{S N R}\right\} .
$$

From Eq. (8), it is also straightforward to infer the OP corresponding to the scenario, in which the received signals experience only fast fading but no block fading or only block fading but no fast fading.

For the sake of providing a fair comparison between different modulation schemes, for example BPSK and QPSK, we should use the normalized throughput of

$$
R_{n}=\frac{R}{\eta} .
$$




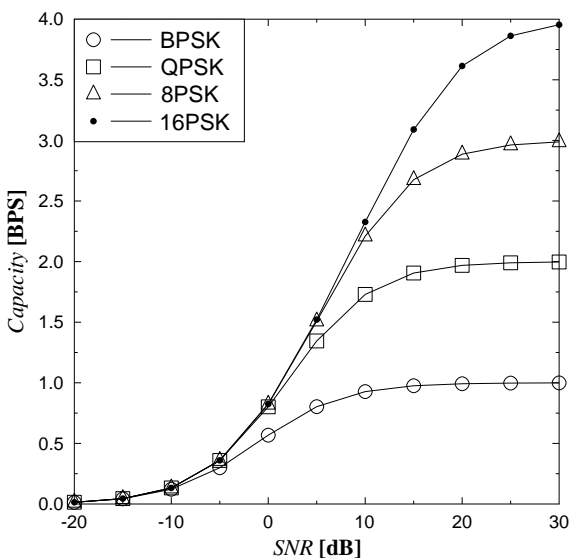

Fig. 4. Capacity curves [118] of the DCMC channel calculated for uncorrelated Rayleigh (small-scale) fading channel, when employing Binary Phase-Shift Keying (BPSK), Quadrature Phase-Shift Keying (QPSK), 8 level Phase Shift Keying (8PSK) and 16 level Phase Shift Keying (16PSK) schemes.

\begin{tabular}{|c|c|c|}
\hline Normalised throughput & \multicolumn{2}{|c|}{$S N R_{r} \mid R_{n}$ [dB] } \\
\hline$R_{n}=R / \eta$ & BPSK $(\eta=1)$ & QPSK $(\eta=2)$ \\
\hline \hline 0.01 & -21.45 & -18.55 \\
\hline 0.20 & -7.38 & -4.40 \\
\hline 0.40 & -3.01 & -0.01 \\
\hline 0.50 & -1.20 & 1.78 \\
\hline 0.60 & 0.59 & 3.60 \\
\hline 0.80 & 4.92 & 7.95 \\
\hline 0.99 & 28.37 & 31.75 \\
\hline
\end{tabular}

TABLE III

$\left.S N R_{r}\right|_{R}$ VALUES OF EQ. (8) FOR DCMC SCHEMES CORRESPONDING TO CONSIDERING DIFFERENT NORMALISED THROUGHPUTS, NAMELY

$R_{n}=\{0.01,0.2,0.4,0.5,0.6,0.8,0.99\}$, WHEN COMMUNICATING OVER UNCORRELATED RAYLEIGH FADING CHANNELS.

Accordingly, the $\left.S N R_{r}\right|_{R}$ values corresponding to different values of the normalised throughput $R_{n}$ considered in the DCMC scheme can be calculated and listed in Tab. III. Accordingly, we have Fig. 5 characterising the relationship between the OP versus the normalised throughput $R_{n}$, when employing the BPSK and QPSK modulation schemes for transmission over slow Rayleigh fading channel.

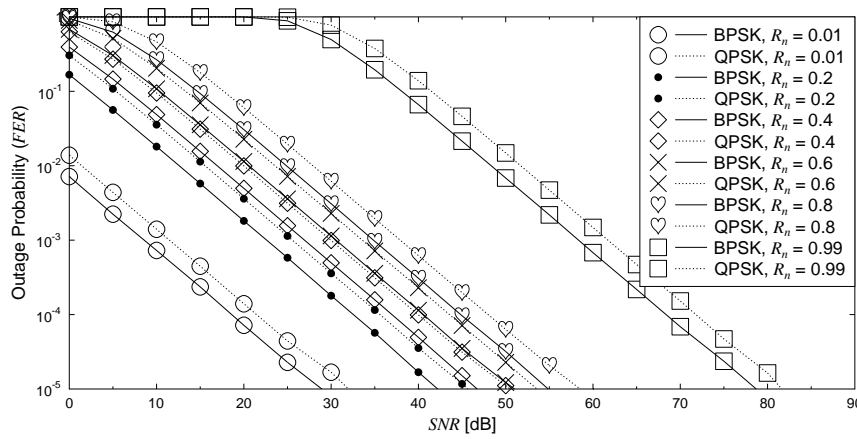

Fig. 5. Outage probability (FER) of the DCMC calculated by Eq. (8) for BPSK and QPSK modulation scheme for transmission over the largescale(slow) Rayleigh fading channel.

Additionally, the DCMC capacity can also be calculated by employing the EXIT chart method, which is illustrated in the next section for determining the capacity of the D-DCMC.

\section{Differential Discrete-Input Continuous-Output Memoryless Channel}

EXIT charts may be invoked for calculating the D-DCMC capacity supported by the non-coherent detection scheme of Section II-D. More specifically, it can be inferred from the second property of the EXIT chart detailed in Section II-A2 that the area under the EXIT curve of a Differential Quadrature Phase Shift Keying (DQPSK) modem for example quantifies its attainable capacity. Accordingly, in order to obtain the capacity curves seen in Fig. 6, the following steps have to be carried out:

- Generate the EXIT curves of various differential modulation schemes, for example those of Differential Binary Phase Shift Keying (DBPSK) and DQPSK, for different $S N R_{r}$ values.

- Determine a single point on the capacity curves by computing the area under an EXIT curve and its corresponding $S N R_{r}$. As a result, the capacity curves are plotted in Fig. 6 for an uncorrelated Rayleigh fading channel, when for example employing DBPSK and DQPSK modulation schemes.

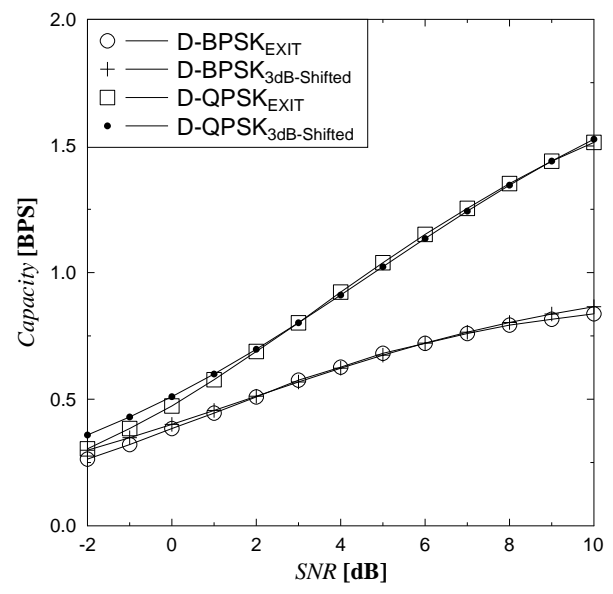

Fig. 6. Capacity curves of the D-DCMC determined by the EXIT-chart based method or by shifting the DCMC capacity curves of Fig. 4 to the right a distance of $3 \mathrm{~dB}$, when employing BPSK and QPSK modulation schemes over uncorrelated Rayleigh (fast) fading channel.

As an alternative method, the D-DCMC capacity curves can be obtained by shifting the corresponding DCMC capacity curves plotted in Fig. 4 to the right by $3 \mathrm{~dB}$. As a result, the D-DCMC curves determined by the shifting method are also plotted in Fig. 6.

Having obtained the D-DCMC capacity curves, at a given throughput of $R$, the $\left.S N R_{r}^{E X I T}\right|_{R}$ required for maintaining this specific throughput can now be specified, again as seen in Fig. 6. Note that the normalised throughput $R_{n}$ should be used for the sake of a fair comparison. Accordingly, the $\left.S N R_{r}^{E X I T}\right|_{R}$ values are determined and listed in Tab. IV for different values of the normalised throughput $R_{n}$.

Then, similar to the OP of the DCMC channel formulated in Eq. (8) the term of $\left.S N R_{r}\right|_{R}$ in Eq. (8) should be replaced by that of $\left.S N R_{r}^{E X I T}\right|_{R}$, in order to construct an expression for the OP corresponding to the D-DCMC model. Accordingly, the OP of the scenario pertaining to the uncorrelated Rayleigh 


\begin{tabular}{|c|c|c|}
\hline Normalised throughput & \multicolumn{2}{|c|}{$\left.S N R_{r}^{E X I T}\right|_{R_{n}}[\mathbf{d B}]$} \\
\hline$R_{n}=R / \eta$ & DBPSK $(\eta=1)$ & DQPSK $(\eta=2)$ \\
\hline \hline 0.01 & -12.03 & -10.31 \\
\hline 0.20 & -3.13 & -0.78 \\
\hline 0.40 & 0.26 & 2.98 \\
\hline 0.60 & 3.44 & 6.45 \\
\hline 0.80 & 7.89 & 11.32 \\
\hline 0.99 & 28.00 & 32.00 \\
\hline
\end{tabular}

TABLE IV

$\left.S N R_{r}^{E X I T}\right|_{R_{n}}$ VALUES CALCULATED WITH THE AID OF THE EXIT CHARTS TO BE INTRODUCED AT A LATER STAGE IN SECTION V FOR A D-DCMC SCHEME, WHEN VARIOUS NORMALISED THROUGHPUT $R_{n}$, NAMELY $0.01,0.2,0.4,0.6,0.8$ AND 0.99 , ARE CONSIDERED.

(fast) and the block Rayleigh (slow) fading channels can be quantified. For example, Fig. 7 visualises the resultant OP associated with diverse values of the normalised throughput $R_{n}$ for the block Rayleigh fading channel, when for example DBPSK and DQPSK modulation schemes are employed.

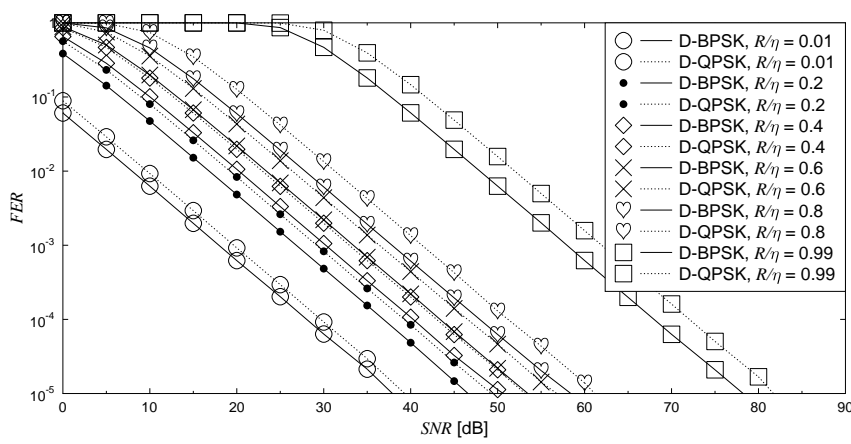

Fig. 7. Outage probability $(F E R)$ of the D-DCMC system operating over block Rayleigh fading channels, when DBPSK and DQPSK modulation schemes are used. The OP is plotted across different values of the normalised throughput $R / \eta$, namely $0.01,0.2,0.4,0.6,0.8,0.99$.

It should be noted that the EXIT chart based method applied for the D-DCMC based system can also be used for the DCMC based system and can provide similar results to those obtained by the formula provided in [118], as detailed in Section III-B. Thus, EXIT charts can be considered to be an generic tool for evaluating the OP and the outage capacity of an arbitrary coded modulation scheme. We will further clarify this aspect in the subsequent sections.

\section{Outage CAPacity}

The concept of the outage capacity [116], [119]-[121] was shown to be useful for representing the best possible performance of a system, namely the SNR-versus-FER performance, where a given degree of QoS quantified in terms of the FER is required. This upper bound of the system's performance is useful for designing a specific system.

Let us consider a scenario, where the channel coding is unable to significantly improve the achievable error probability in block fading channels, as elaborated on in [116]. More specifically, in the presence of the small-scale fading, where the channel coefficients change for every symbol, the transmitter can send data at the rate of $R<\left.C\right|_{h}$, while maintaining an arbitrarily low error probability. However, it may not be possible to maintain a vanishingly low BER for a large-scale fading channel, where the channel coefficients remain constant over a frame duration. Thus, the capacity of large-scale fading channels may become zero in the strict sense.

Hence, the outage capacity $C(\varepsilon)$ introduced in [116], [119] was defined as the highest possible value of transmission rate $R$, which was still capable of ensuring that the OP or FER becomes less than $\varepsilon$. It should be noted that the capacity of the quasi-static fading channels is dependent on the OP $\varepsilon$ characterising the desired quality of transmission. Thus, the outage capacity is different from the channel capacity detailed in Section III-B and Section III-C.

Similar to the formulaic relationship established for the CCMC case in [116], it can be inferred from (8) that the outage capacity of the DCMC channel may be formulated as:

$$
\operatorname{SNR}(\varepsilon, \eta)=F^{-1}(1-\varepsilon),
$$

where $F\left(\left.S N R_{r}\right|_{R}\right)$ is the Complementary Cumulative Distribution Function (CCDF) of the combined fast-and slow-fading (small-and large scale-fading) envelope of $|h|^{2}=\left|h_{s}\right|^{2}\left|h_{f}\right|^{2}$, which is defined as

$$
F\left(\left.S N R_{r}\right|_{R}\right)=\operatorname{Pr}\left\{\left|h_{s}\right|^{2}>\frac{\left.S N R_{r}\right|_{R}}{S N R}\right\} .
$$

Note that $F\left(\left.S N R_{r}\right|_{R}\right)$ depends on the transmission rate $R$, while the corresponding $S N R_{r}$ may be calculated by the method illustrated in Section III-B and Section III-C, where the EXIT chart tool is considered as a generic tool. In order to provide a fair comparison of the different-thoughput modulation schemes, the CCDF $F\left(\left.S N R_{r}\right|_{R_{n}}\right)$ of the $S N R_{r}$ is used at the same value of the normalised transmission rate $R_{n}$. Accordingly, the transmission rate of Eq. (11) should be replaced by the normalised transmission rate $R_{n}$.

\section{A. Coherent schemes}

For the outage capacity of the DCMC system, let us first recall the DCMC capacity presented in Section III-B. Again, at a given normalised throughput of $R_{n}$, we can readily identify the receive signal to noise power ratio $\left.S N R_{r}\right|_{R_{n}}$ required for maintaining $R_{n}$ from the DCMC capacity curves, namely those plotted in Fig. 4. As a result, the $\left.S N R_{r}\right|_{R_{n}}$ values corresponding to the normalized throughput $R_{n}$ are obtained and summarised in Tab. III. The $\left.S N R_{r}\right|_{R_{n}}$ values of Tab. III are employed for computing the CCDF of $F\left(\left.S N R_{r}\right|_{R_{n}}\right)$ defined by Eq. (11).

Having computed the CCDF of $F\left(\left.S N R_{r}\right|_{R_{n}}\right)$, it is now ready to calculate the minimum value of the $S N R$ required for achieving a given OP of $\eta$ by applying Eq. (10). For the sake of comparing different modulation schemes, the notation $C_{n}(\varepsilon)$ is defined as the normalised outage capacity, which is given by

$$
C_{n}(\varepsilon)=\frac{C(\varepsilon)}{\eta}
$$

where $C(\varepsilon)$ is the outage capacity determined by the relationship of Eq. (10), while $\eta$ is the number of modulated bits used for representing a symbol in the modulation scheme of consideration. 
As a result, the normalised outage capacity $C_{n}(\varepsilon)$ of Eq. (12) can be calculated, when the OP of $\varepsilon$ is known. For example, the curves representing the outage capacity are plotted in Fig. 8 for a range of OP values $\varepsilon$, namely for $\varepsilon=10^{-1}, 10^{-2}, 10^{-3}$, when the BPSK and the QPSK modulation schemes are employed in the block Rayleigh fading channel.

Let us now exemplify the above-mentioned steps by considering a specific system employing the parameters summarised in Tab. V.

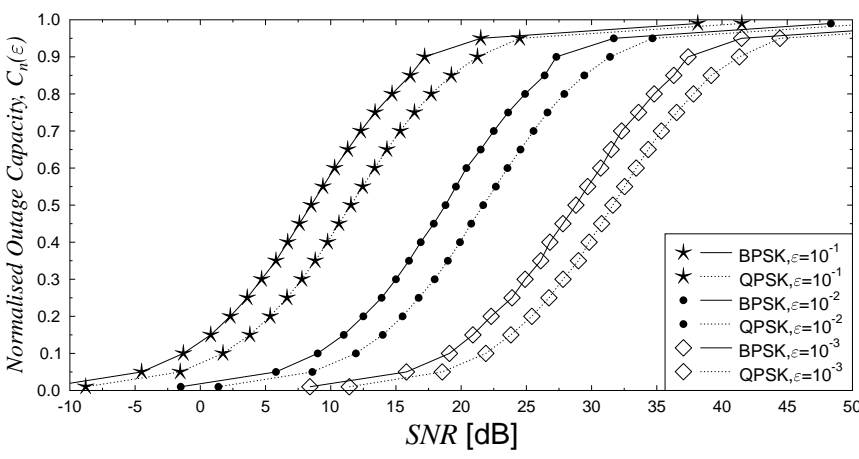

Fig. 8. Outage capacity of the DCMC calculated by Eq. (10) upon the CCDF of $F\left(\left.S N R_{r}\right|_{R}\right)$ defined by Eq. (11), for the range of the OP $\eta$, namely $10^{-1}, 10^{-2}$ and $10^{-3}$, when the BPSK and the QPSK modulation schemes are employed for communicating over the block Rayleigh fading channel.

\begin{tabular}{|l|l|}
\hline Parameters & \multicolumn{1}{|c|}{ Values } \\
\hline \hline Transmission rate & $R=1[$ Bit Per Symbol (BPS)] \\
\hline Modulation scheme & QPSK \\
\hline Modulation lever & $L=4$ \\
\hline Number of bit per symbol & $\eta=2$ bits \\
\hline Equivalent channel coding rate & $R_{c}=0.5$ \\
\hline Required OP & $\varepsilon=10^{-3}$ \\
\hline
\end{tabular}

TABLE V

THE RELATING PARAMETERS OF THE ILLUSTRATIVE SYSTEM EMPLOYING QPSK MODULATION (NUMBER OF MODULATION LEVELS $L=4$ AND NUMBER OF MODULATED BITS PER SYMBOL $\eta=2$ ) AND A CHANNEL CODING SCHEME HAVING A CODING RATE $R_{c}=0.5$, WHICH RESULTS IN THE INFORMATION RATE $R=1.0$. THE OUTAGE CAPACITY CORRESPONDING TO THE OP $\varepsilon=10^{-3}$ IS CALCULATED UPON THE LISTED PARAMETERS.

Since a QPSK modulation scheme is employed in the example considered, we can have $\eta=2$ and $L=2^{2}=4$ either as inputs for the channel capacity formulae of DCMC capacity detailed in [118] or as parameters for estimating the corresponding channel capacity using the EXIT chart based method advocated. The resultant capacity curve is shown in Fig. 9. Accordingly, given the transmission rate of $R=R_{c} \eta=0.5 \times 2.0=1.0$, the associated signal-to-noise ratio of $\left.S N R_{r}\right|_{R}=1.793 \mathrm{~dB}$ can be determined in Fig. 9 . Having computed the CCDF of $F\left(\left.S N R_{r}\right|_{R}=1.793\right)$ plotted in Fig. 10, we can then compute the minimum $S N R$ required for maintaining a given OP of $\varepsilon=10^{-3}$ by substituting $\eta=2$ and $\varepsilon=10^{-3}$ into Eq. (10). As a result, the $S N R=31.63 \mathrm{~dB}$ shown in Fig. 10 corresponds to an OP of $\varepsilon=10^{-3}$. Naturally, the point having $R=1.0$ BPS and $S N R=31.63 \mathrm{~dB}$ constitutes a single point on the outage capacity curve plotted in Fig. 11. Repeatedly, other $S N R$ values associated with different values of $R$ are calculated in order to form the outage capacity curve plotted in Fig. 11, provided that the same OP of $\varepsilon=10^{-3}$ is considered.

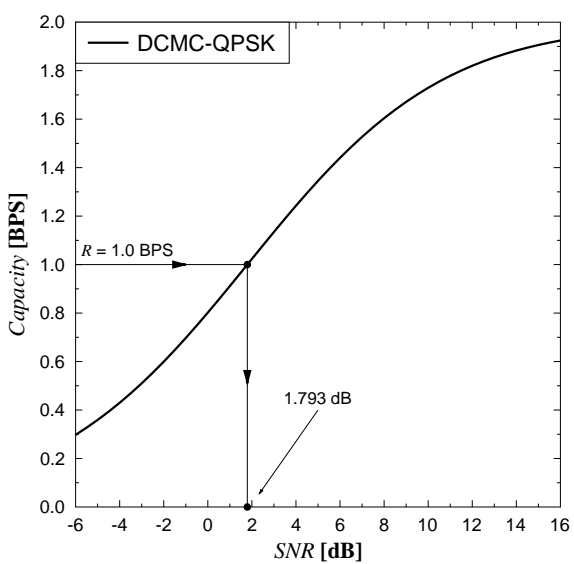

Fig. 9. The DCMC capacity curve for small-scale Rayleigh fading channel, when the QPSK modulation is employed. The value of $\left.S N R_{r}\right|_{R}$ corresponding to $R=R_{c} \eta=0.5 \times 2.0=1.0$ is specified on the DCMC capacity curve.

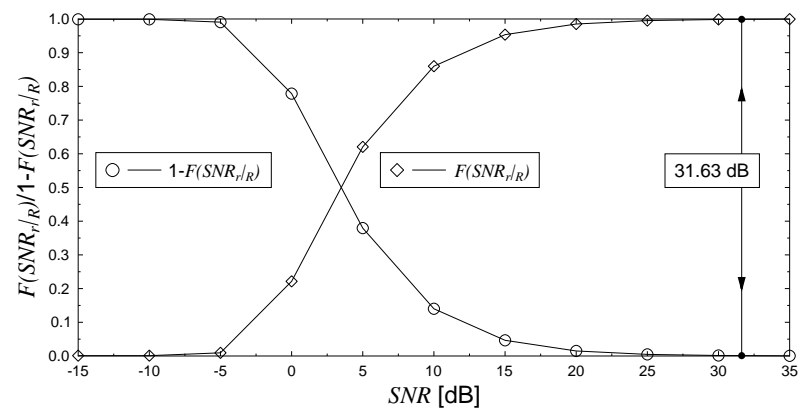

Fig. 10. The CCDF of $F\left(\left.S N R_{r}\right|_{R}\right)$ given in Eq. (11) and its offset function of $\left(1-F\left(\left.S N R_{r}\right|_{R}\right)\right)$ are calculated across different values of $S N R$ for the value of $\left.S N R_{r}\right|_{R}=1.793$, which is associated with the specific system having the relevant parameters listed in Tab. V.

In brief, the specific point at $R=1.0$ BPS and $S N R=$ $31.63 \mathrm{~dB}$ for $\varepsilon=10^{-3}$ recorded in Fig. 11 may be interpreted as follows. If the $S N R_{r}$ value is equal to or lower than $31.63 \mathrm{~dB}$, the maximum outage capacity is equal to $1.0 \mathrm{BPS}$, provided that the OP of $\varepsilon=10^{-3}$ is guaranteed. Viewed from a different perspective, if we have $S N R_{r} \leq 31.63 \mathrm{~dB}$ at the receiver and the transmission rate is $R=1$ BPS, the best possible quality of transmission is achieved at an OP of $\varepsilon=10^{-3}$.

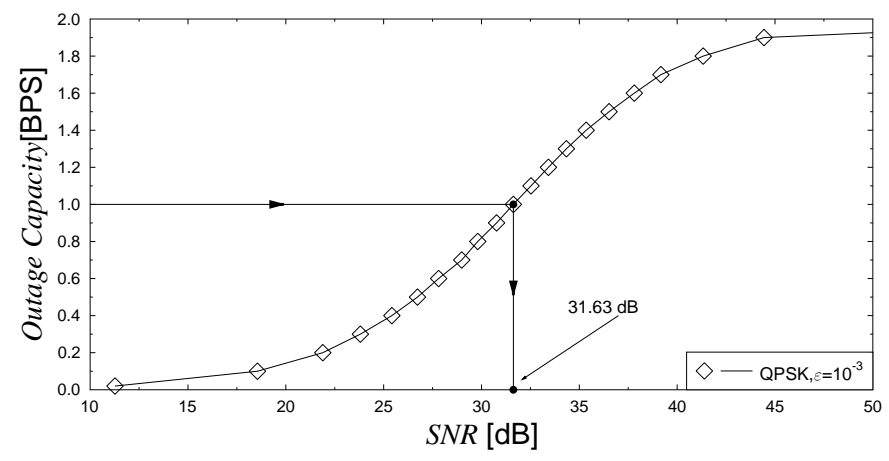

Fig. 11. The outage capacity of Eq. (11) corresponding to the OP value of $\varepsilon=10^{-3}$ for the DCMC system characterised by Tab. V. 


\section{B. Non-coherent schemes}

It should be noted that the general outage capacity formula of Eq. (10) can also be used for the D-DCMC case, where the CCDF $F\left(\left.S N R_{r}\right|_{R}\right)$ of the achievable rate $R$ is replaced by $F\left(\left.S N R_{r}^{E X I T}\right|_{R}\right)$, which can be obtained by substituting the value of $\left.S N R_{r}\right|_{R}$ in Eq. (11) by that of $\left.S N R_{r}^{E X I T}\right|_{R}$.

Note that the CCDF $F\left(\left.S N R_{r}^{E X I T}\right|_{R}\right)$ depends both on the transmission rate $R$ and on the modulation scheme employed. The two parameters are used for specifying the $\left.S N R_{r}^{E X I T}\right|_{R}$ value on the corresponding capacity curve calculated by the EXIT-chart based method, as detailed in Section III-C, where the capacity curves of DBPSK and DQPSK are plotted in Fig. 6. Similarly to the DCMC case, the normalised transmission rate $R_{n}$ should be employed for the sake of appropriately characterising different modulation schemes, such as DBPSK and DQPSK. Hence, the term $\left.S N R_{r}^{E X I T}\right|_{R}$ is substituted by the term $\left.S N R_{r}^{E X I T}\right|_{R_{n}}$.

Accordingly, having calculated $\left.S N R_{r}^{E X I T}\right|_{R_{n}}$ values listed in Tab. IV, the CCDF $F\left(\left.S N R_{r}^{E X I T}\right|_{R_{n}}\right)$ similarly presented by Eq. (11) is obtained. Then, the CCDF $F\left(\left.S N R_{r}^{E X I T}\right|_{R_{n}}\right)$ can be used in conjunction with Eq. (10) for calculating the outage capacity of a perfect D-DCMC capacity-achieving system. For the sake of comparison between different modulation schemes, namely DBPSK and DQPSK, the normalised outage capacity $C_{n}(\varepsilon)$ defined by Eq. (12) is utilised for characterising the outage capacity of a perfect D-DCMC capacityachieving system. As a result, we have Fig. 12 showing the normalised outage capacity pertaining to a range of OP values $\varepsilon$, namely to $\varepsilon=10^{-1}, 10^{-2}$ and $10^{-3}$, when the DBPSK and DQPSK modulation schemes are used for communicating over the block Rayleigh fading channel.

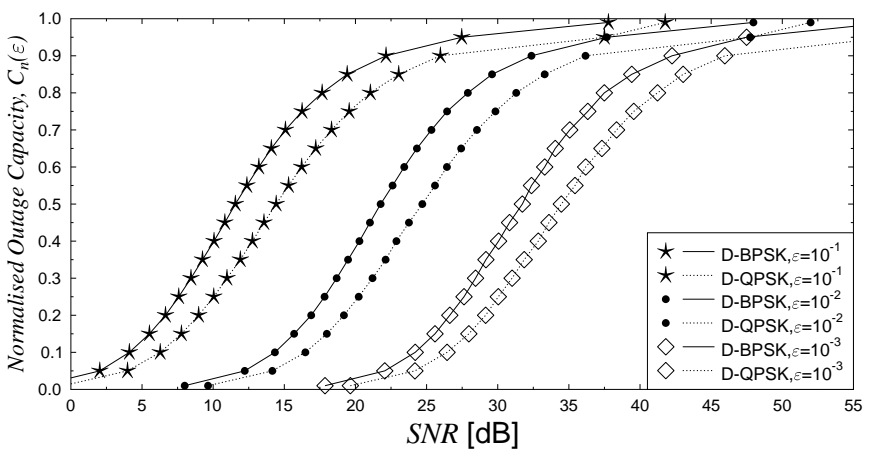

Fig. 12. The normalised outage capacity $C_{n}(\varepsilon)$ of the D-DCMC associated with the range of $\mathrm{OP}$ values $\varepsilon$, namely with $\varepsilon=10^{-1}, 10^{-2}$ and $10^{-3}$, is calculated by for the cases of employing the DBPSK and the DQPSK modulation schemes, when communicating over the block Rayleigh fading channel.

\section{NEAR-CAPACITY CHANNEL CODE DESIGN}

As portrayed in Fig. 2 about the architecture of our tutorial, after having detailed both OP and outage capacity, which can be served as benchmarkers for the EXIT-chart based nearcapacity design, we are now at a convenient position to present the design principles with the aid of illustrative examples.

\section{A. EXIT-chart based code design principles}

The design principles that we present here are based on exploiting the basic properties of EXIT charts, which are detailed in Section II-A. According to the first property, EXIT charts can be used for visually observing the flow of soft-information exchanges. Hence, the benefits of iterations between the components of the composite coding schemes may be comprehensively visualised by diagrams. As a result, both 3D-EXIT [39], [122]-[124] and 2D-EXIT [44], [125], [126] charts have been shown to be helpful in investigating complex composite coding schemes.

The second property of EXIT charts [31], [77], [85] is that the area under the EXIT curve of an inner decoder component reflects the corresponding channel capacity. Accordingly, the designer is able to measure and compare the different channel capacities in order to facilitate the design of composite coding schemes as well as to determine the SNR-distance from the capacity of a specific coding scheme.

In the context of irregular code designs [44], [45], [125], [126], the irregular outer code employed is capable of adjusting the shape of its EXIT curve to match that of the corresponding inner amalgamated code. In such irregular codes, the IrCC may be used as a typical example of the outermost coding stage of a serially concatenated scheme. Based on a number of designs using irregular codes [44], [45], [125], [126], we summarise the associated design guidelines in the form of the following two-step procedure:

- Step1: Create the EXIT curve of the amalgamated inner decoder. The amalgamated inner decoder can be constituted by multi-component schemes, for example by the above-mentioned URC having a beneficial IIR with the inner-most detector constituted by the demodulator. An appropriate number of iterations between the components, for example the number of decoding iterations between the URC decoder and the inner-most detector, is decided based on exploiting the second properties of the EXIT charts. More specifically, the area under the EXIT curve of this amalgamated inner decoder represents the respective achievable DCMC capacity [31]. Naturally, a high number of iterations would incur a high complexity, the design objective is to opt for the minimum number of iterations, which only result in a marginal capacity loss.

- Step2: Carefully choose the overall coding rate of the outer IrCC encoder in order to match it to the shape of the amalgamated inner decoder. The EXIT curve matching algorithm of [30] may then be employed for searching for the most appropriate configuration of the outer code. The matching algorithm aims for finding a set of codes facilitating decoding convergence to a vanishingly low $B E R$ at the lowest possible $S N R_{r}$, where a narrow but marginally open EXIT chart tunnel would appear in the EXIT chart.

In what follow, the general principle of the two-step procedure briefly above-described will be demonstrated with the aid of specific design examples detailed in Section V-B, Section V-C and Section VI-C. As visualised in the architecture of the manuscript portrayed in Fig. 2, the specific design examples of Irregular Convolutional Coded Unity Rate Coded M-ary Phase Shift Keying (IrCC-URC-MPSK), Irregular Convolutional Coded Unity Rate Coded Differential M- 
ary Phase Shift Keying (IrCC-URC-DMPSK) and Irregular Convolutional Coded Unity Rate Coded Space Time Trellis Coded M-ary Phase Shift Keying (IrCC-URC-STTC-MPSK) coding arrangements will be respectively presented.

\section{B. IrCC-URC-MPSK coding scheme}

In this section, the design principles summarised in Section V-A are illustrated by a specific design example, namely those of a coherent-detection based scheme constituted by the near-capacity IrCC-URC-MPSK scheme, which may be used in a transmission system portrayed in Fig. 13.

At the transmitter side seen in Fig. 13, an information frame of $N$ bits is encoded by the IrCC encoder having a coding rate of $R_{c}$, in order to produce an output frame having a frame length of $N / R_{c}$ bits. The frame is then interleaved by the interleaver $\pi$ before being encoded again by the URC encoder for providing a URC-encoder frame of the same length, which is of $N / R_{c}$ bits. The frame from output of the URC encoder is modulated by a modulation scheme employing $\eta$ bits for representing a symbol, before transmitting to the receiver side, as seen in Fig. 13.

At the receiver side pictured in Fig. 13, the signals received during a single frame duration are demodulated then decoded by the URC decoder before entering the iteratively decoding process of $J$ iterations occurring between the inner decoder and the outer IrCC decoder, where the interleaver $\pi$ and deinterleaver $\pi^{-1}$ are employed in each iteration. The inner decoder component is an amalgamated arrangement comprising the demodulation block and the URC decoder, as seen in Fig. 13.

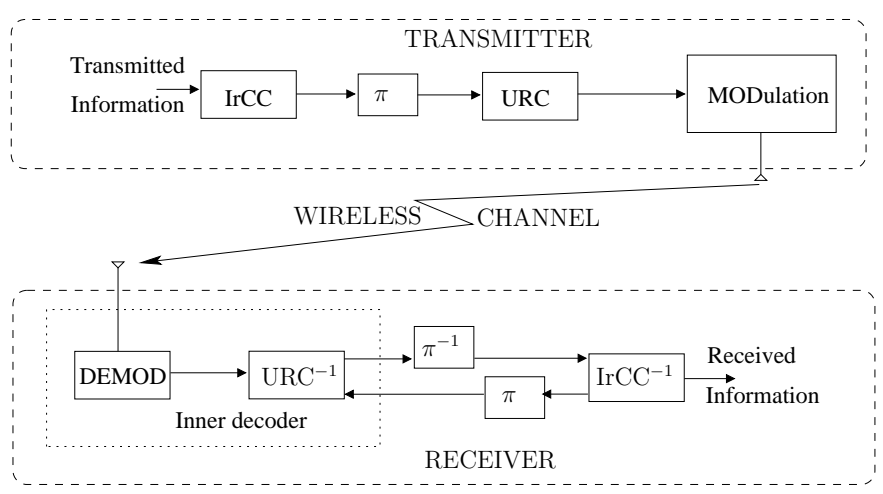

Fig. 13. Architecture of a transmission system employing IrCC-URC-MPSK scheme.

1) EXIT-charts matching and optimisation: By invoking the EXIT chart-aided design principles presented in Section V-A, the specific design for IrCC-URC-QPSK scheme can be summarised as follows:

Step1: Create the EXIT curve of the inner decoder constituted by the Unity-Rate Code and Quadrature Phase-Shift Keying (URC-QPSK) scheme for different $S N R_{r}$ values for respective outer codes, namely $S N R_{r}=3.6,3.4,3.2,3.0$ and $2.8 \mathrm{~dB}$ for the 17-subcode IrCC in Fig. 14, as well as namely $S N R_{r}=2.7,2.6,2.5,2.4$ and $2.3 \mathrm{~dB}$ for the 36-subcode IrCC in Fig. 15. The two outer codes of both the 17 -subcode

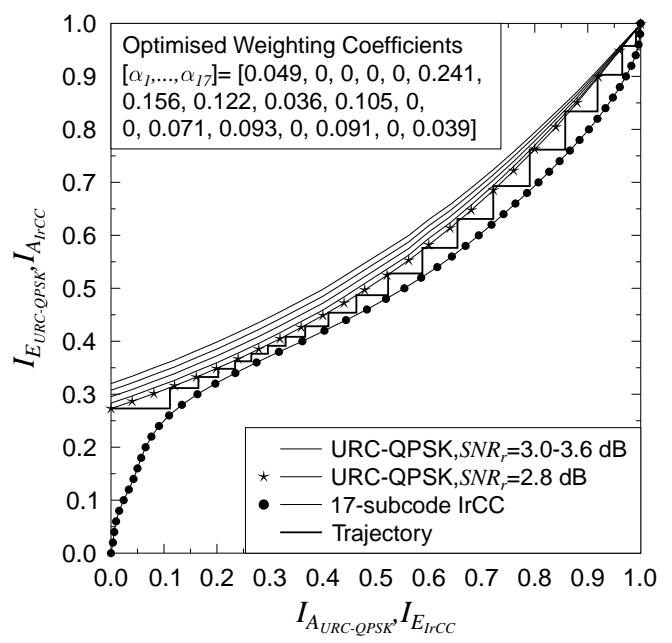

Fig. 14. The EXIT curves of the inner decoder URC-QPSK and the outer decoder 17-subcode IrCC for a single transmission link along with the MonteCarlo simulation based decoding trajectory, provided that $R_{c}=0.5$.

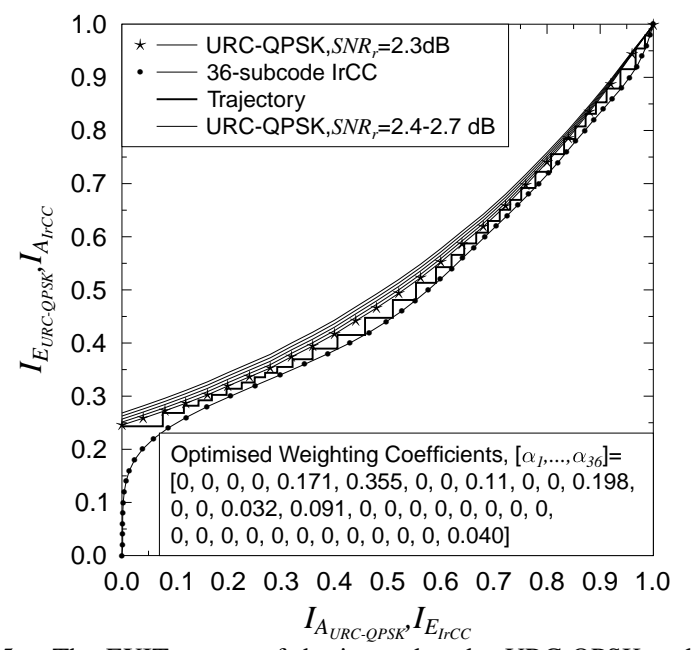

Fig. 15. The EXIT curves of the inner decoder URC-QPSK and the outer decoder 36-subcode IrCC having the coding rate $R_{c}=0.5$ for a single transmission link along with the Monte-Carlo simulation based decoding trajectory.

IrCC and of the 36-subcode IrCC are presented in parallel in Tab. VI for highlighting how a near-capacity performance can be achieved by adjusting the configuration of the outer code, namely by adjusting the number and weight of the subcodes.

It shown by Fig. 16 that there is no iterations within the inner decoder component, since no improvement in the capacity is seen when performing iterative decoding at the inner decoder of URC-QPSK. More specifically, it is seen in Fig. 16 that the capacity of the URC-QPSK scheme almost coincides with the DCMC-QPSK curve, which confirms that no iterations between URC and QPSK demodulator are required. Hence, a narrow tunnel is created between the EXIT curve of the outer IrCC decoder and the inner URC-QPSK decoder is sufficient to ensure that a near-capacity coding scheme is conceived.

Step2: The system throughput is set, for example to $R=1$. Then, given the IrCC code rate of for example $R_{c}=0.5$, 
employing the EXIT curve matching algorithm of [30] results in the optimised weighting coefficients $\alpha_{i}, i=1, \ldots, 17$ ( $i=$ $1, \ldots, 36)$ of the 17 (36) different-rate subcode IrCC codes. The exact values of theses coefficients for the 17-subcode IrCC code and the 36-subcode IrCC code can be found in Fig. 14 and Fig. 15, respectively, as well as in Tab. VI.

It is plausible that the more subcodes used to form an IrCC encoder, the better its EXIT curve can match to the inner coding arrangement. This implies that the IrCC-URCMPSK scheme employing the IrCC having 36 subcodes is capable of performing more closely to the corresponding DCMC capacity than the IrCC having 17 subcodes. These conclusions are supported by the different EXIT chart tunnel areas seen in Fig. 14 and Fig. 15. For example, as a result of the 36-subcode based IrCC (36co-IrCC) capable of providing a more flexible EXIT curve, its EXIT curve matches more closely to the EXIT curve of inner components than EXIT curve of 17-subcode IrCC (17co-IrCC). As suggested by Fig. 15, the 36co-IrCC-URC-QPSK scheme performs only about $(2.3-1.8)=0.5 \mathrm{~dB}$ away from the DCMC-QPSK, provided that $J=40$ outer iterations is invoked. The result implies that the higher complexity we can afford, the closer the coding scheme can operate to the corresponding DCMC capacity. As shown in the literature of EXIT-chart aided designs, a low distance of $0.3 \mathrm{~dB}$ to the corresponding capacity can be attained, if the component codes are carefully chosen [77] for ensuring that the matching of EXIT curves [40] is sufficiently accurate.

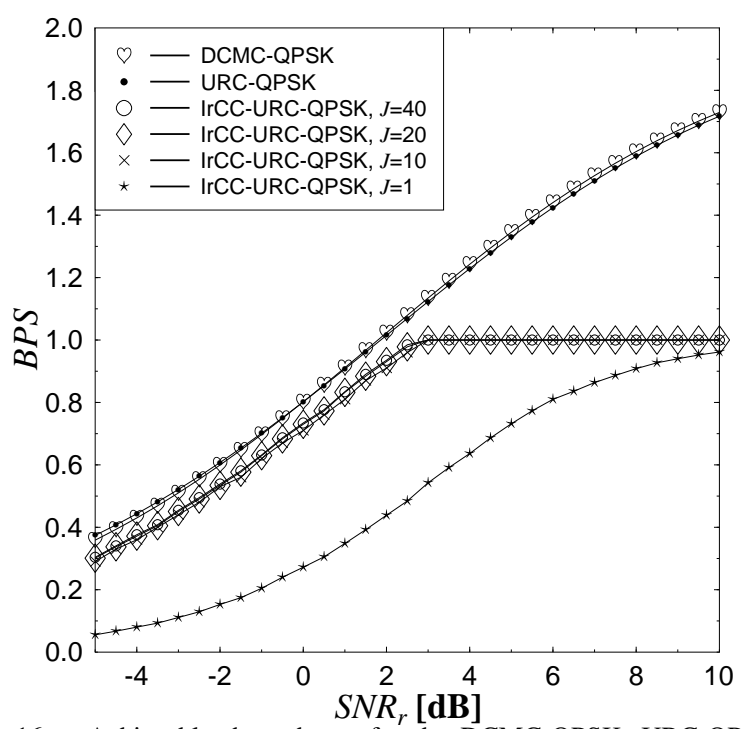

Fig. 16. Achievable throughputs for the DCMC-QPSK, URC-QPSK and IrCC-URC-QPSK based systems, when communicating over Rayleigh smallscale fading channel.

2) Performance of IrCC-URC-MPSK coding scheme: The accuracy of the system performance predicted by the EXIT chart-aided design can be confirmed by Monte-Carlo simulations compared to the corresponding channel capacities estimated with the aid of the EXIT chart's properties.

The performance curves presented in Fig. 17 convey the clear message that a near-capacity performance is achieved at the cost of an additional complexity quantified in terms of the number of iterations required. Fig. 17 shows that when a higher number of iterations is applied, a nearer-to-capacity performance can be observed. If $J=40$ iterations were affordable for the case of the 36-subcode IrCC, the corresponding trajectory would reach the $(1,1)$ point in Fig. 15, which guarantees a vanishingly low $B E R$.

To further appraise the design from another perspective, the area property of EXIT-charts detailed in Section II-A2 can be exploited for determining the achievable DCMC capacities of the DCMC-QPSK, URC-QPSK and IrCC-URC-QPSK systems, which are quantified in Fig. 16. More specifically, it is seen in Fig. 16 that the capacity of the URC-QPSK scheme almost coincides with the DCMC-QPSK curve, which confirms that the performance of the URC-QPSK scheme has indeed reached the attainable capacity of DCMC-QPSK. Fig. 16 also shows the achievable channel capacity improvements corresponding to $J=1,10,20$ and 40 iterations. There is only a negligible further improvement for using $J=40$ in comparison to $J=20$.

The same procedure may be replicated for other modulation schemes, namely 8PSK and 16PSK, in order to obtain the corresponding weighting coefficients listed in Tab. VI, which stipulate the configurations of the outer codes of IrCC. The turbo-cliff $T_{u}$ detailed in Tab. VI represents the $S N R$ values where the coding schemes may operate with an infinitesimally low BER. Accordingly, $D_{c}$ representing the distance to a respective DCMC capacity in Tab. VI was calculated upon $T_{u}$ and the value of $S N R$ corresponding to the channel capacity detailed in Section III-B.

\begin{tabular}{|l|r|}
\hline $\begin{array}{l}\text { Arrangement } \\
\text { Turbo-cliff }\left(T_{u}\right) \\
\text { Distance to capacity }\left(D_{c}\right)\end{array}$ & Coefficients: $\left[\alpha_{1}, \alpha_{2}, \ldots, \alpha_{17}\right]$ \\
\hline URC-QPSK & \\
$T_{u}=2.8 \mathrm{~dB}$ in Fig. 14 & $0.049,0,0,0,0,0.241,0.156$, \\
$D_{c}=1.0 \mathrm{~dB}$ & $0.071,0.093,0,0.091,0,0.039]$ \\
\hline URC-8PSK & {$[0.062,0,0,0,0.247,0.154$,} \\
$T_{u}=5.9 \mathrm{~dB}$ & $0,0,0.041,0.169,0,0.082$, \\
$D_{c}=1.0 \mathrm{~dB}$ & $0.049,0,0.090,0.041,0.064]$ \\
\hline URC-16PSK & {$[0.022,0,0.202,0,0.116$,} \\
$T_{u}=9.0 \mathrm{~dB}$ & $0.073,0,0.061,0.151,0,0$, \\
$D_{c}=1.0 \mathrm{~dB}$ & $0,0.202,0,0,0.023,0.145]$ \\
\hline & Coefficients: $\left[\alpha_{1}, \alpha_{2}, \ldots, \alpha_{36}\right]$ \\
\hline URC-QPSK & {$[0,0,0,0,0.071,0.355,0,0,0.11$,} \\
$T_{u}=2.3 \mathrm{~dB}$ in Fig. 15 & $0,0,0.198,0,0,0.032,0.091,0$, \\
$D_{c}=0.5 \mathrm{~dB}$ & $0,0,0,0,0,0,0,0,0,0,0,0,0$, \\
& $0,0,0,0,0.04]$ \\
\hline URC-8PSK & {$[0,0,0,0.368,0,0,0.072,0.079$,} \\
$T_{u}=5.4 \mathrm{~dB}$ & $0.096,0,0,0.163,0,0,0.09,0$, \\
$D_{c}=0.5 \mathrm{~dB}$ & $0.08,0,0,0,0,0,0,0,0,0$, \\
& $0,0,0,0,0,0,0,0,0,0.048$ \\
\hline URC-16PSK & {$[0,0.1,0.082,0.078,0.049$,} \\
$T_{u}=8.5 \mathrm{~dB}$ & $0.053,0.047,0,0.126,0,0,0.099$, \\
$D_{c}=0.5 \mathrm{~dB}$ & $0,0,0.084,0,0.059,0,0,0,0$, \\
& $0,0,0,0,0,0.104,0,0,0,0$, \\
& $0,0,0,0.052,0.061]$ \\
\hline
\end{tabular}

TABLE VI

SUBCODE WEIGHTING COEFFICIENTS OF THE IRCC17 AND IRCC36 ENCODERS CORRESPONDING TO DIFFERENT INNER COMPONENTS, NAMELY URC-QPSK, URC-8PSK AND URC-16PSK, WHEN COMMUNICATING OVER RAYLEIGH SMALL-SCALE FADING CHANNEL. BOTH THE IRCC17 AND IRCC36 HAVE THE SAME CODING RATE OF $R_{c}=0.5$. 


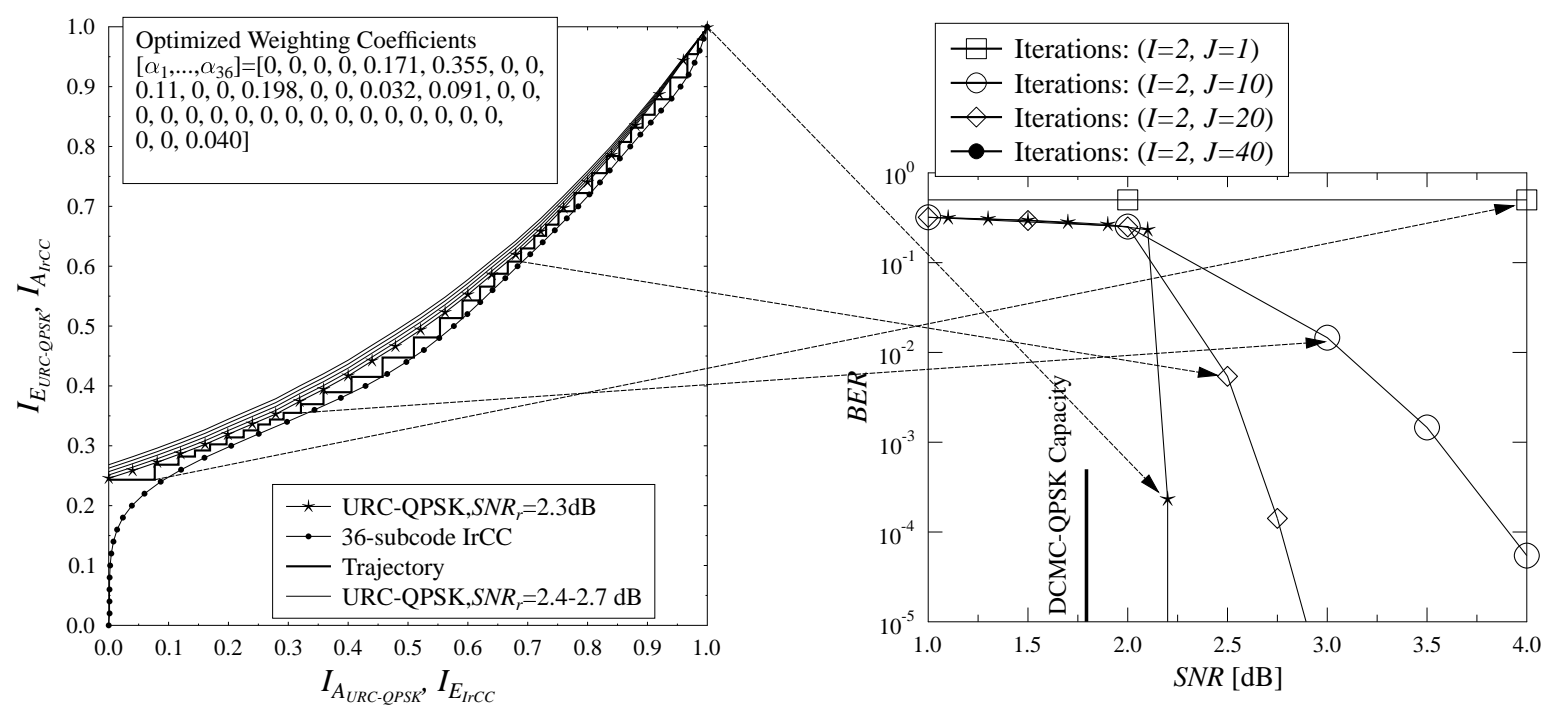

Fig. 17. Performance of the IrCC36-URC-QPSK scheme under Rayleigh small-scale fading conditions, when different numbers of iterations are applied.

\section{IrCC-URC-DMPSK coding scheme}

For the non-coherent based schemes, the EXIT-chart based design principle given in Section $\mathrm{V}$-A can be invoked for facilitating the near-capacity coding design, for example of the IrCC-URC-Multiple-Symbol Differential Detection aided Differential M-ary Phase-Shift Keying (IrCC-URC-MSDDaided-DMPSK) scheme. The scheme may be employed in the system portrayed in Fig. 18.

In the system described by Fig. 18, the transmitted frame of $N$ bits at the transmitter side is first encoded by the IrCC encoder having the coding rate $R_{c}$, in order to produce an egress frame of $N / R_{c}$ bits. The egress frame is mixed by the first interleaver $\pi_{1}$ before being encoded by the URC in order to introduce the resultant frame of the same size. The frame at the output of the URC encoder is again blended by the second interleaver $\pi_{2}$ before being modulated by the differential modulation for transmitting over wireless channel, as seen in Fig. 18.

At the receiver side of the system portrayed in Fig. 18, the signals received from an antenna during a frame duration are first processed in $I$ inner iterations by iteratively performing two steps, namely conducting demodulation using the MSDD method and decoding at the URC decoder. Once having performed the $I$ inner iterations, the resultant frame is fed into $J$ so-called outer iterations. It should be noted that each of $J$ outer iterations contains $I$ inner iterations. As a result, the receiver featured in Fig. 18 has to perform totally $I \times J$ inner iterations, in order to retrieve the transmitted frame.

The primary goal for designing the IrCC-URC-MSDDaided-DMPSK scheme is to determine important parameters stipulating the near-capacity of the systems, namely the optimal values of $I$ inner iterations, $J$ outer iterations as well as configurations of the irregular outer code characterised by weighting coefficients of IrCC. In particular, the EXITchart tool is employed for predicting the number of inner and outer iterations, for formatting the IrCC encoder as well as for determining the D-DCMC capacity of the non-coherent inner most component of the coding scheme, namely the DMPSK and the Unity Rate Coded DMPSK (URC-DMPSK). Moreover, the EXIT chart tool may be used for appraising the design by evaluating the channel capacities associated with different design steps.

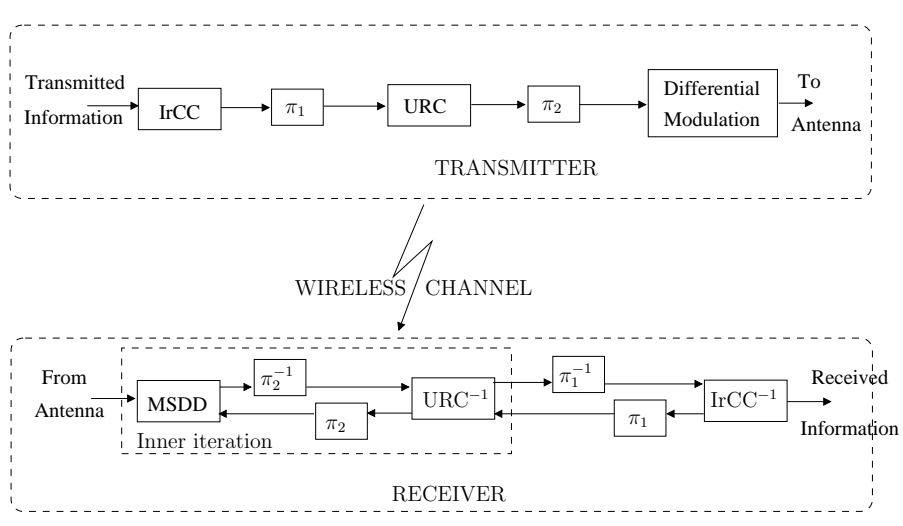

Fig. 18. Architecture of the IrCC-URC-MSDD-aided-DMPSK scheme.

1) EXIT-chart design for IrCC-URC-MSDD-aidedDMPSK: Similar to the manner used for presenting the design example of the coherent scheme in Section V-B, for the sake of clarity, the generic EXIT-chart based design principle presented in Section V-A is demonstrated by applying for a specific example. More specifically, the IrCC-URC-Differential Quadrature Phase Shift Keying (IrCCURC-DQPSK) associated with MSDD $N_{w}=4$ is designed by using the generically applicable EXIT-chart aided method, which is summarised as follows:

Step1: In order to optimise the number $I$ of inner iterations between URC and MSDD-aided DEModulation (MSDDaided-DEM), the area property of EXIT-charts detailed in Section II-A2 can be exploited for determining the achievable capacities of the URC-MSDD-aided-DQPSK and IrCCURC-MSDD-aided-DQPSK systems, which are quantified 
in Fig. 20. In brief, It is shown by Fig. 20 that the capacity curve of the outer scheme (URC-MSDD-aided-DQPSK scheme) approaches that of the inner scheme (the MSDDaided-DQPSK arrangement), when $I>1$ inner iterations are employed for the composite outer decoder of the URC-MSDDaided-DQPSK decoder. It is also demonstrated in Fig. 20 that the attainable capacity improvement becomes negligible for $I>2$. Therefore, the number of inner iterations should be fixed to its optimal value of $I=2$ thereafter;

Step2: Similar to the design procedure presented in Section V-B1. The EXIT curve matching algorithm of [46] can be employed for generating the optimised weighting coefficients $\alpha_{i}, i=1, \ldots, 36$, of the 36 different-rate subcode IrCC codes. As a result, the weighting coefficients of the IrCC associated with $S N R_{r}$ value are obtained, as noted in the upper left side of Fig. 19. Then, these parameters are sufficient to generate the respective trajectory, which exhibits the number $J$ of the outer iterations between IrCC and the amalgamated arrangement URC-MSDD-aided-DQPSK, as seen in the left side of Fig. 19.

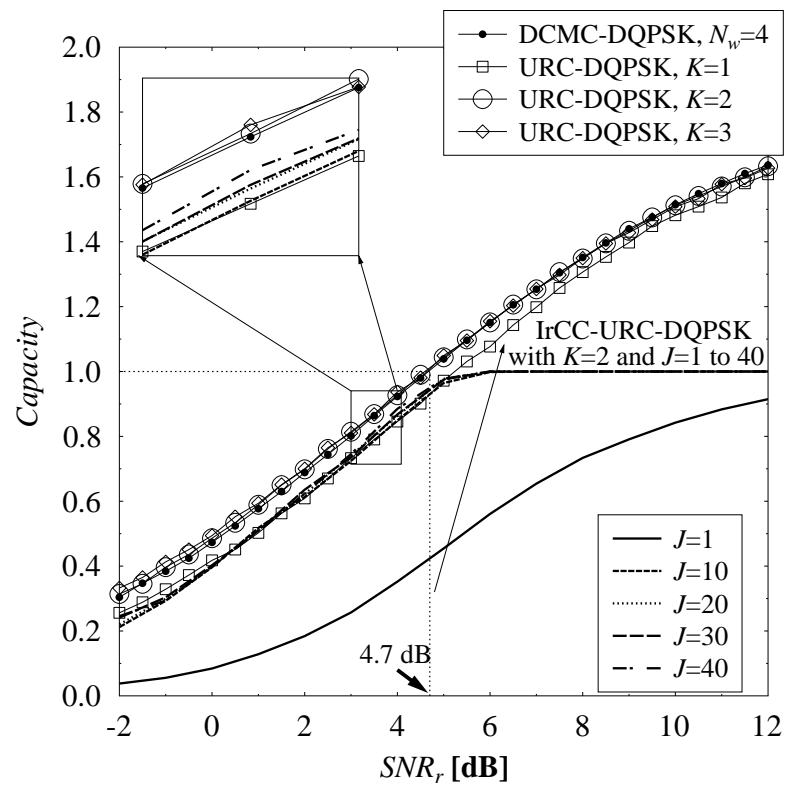

Fig. 20. Channel capacity comparison for the MSDD-aided-DQPSK, URCMSDD-aided-DQPSK and IrCC-URC-MSDD-aided-DQPSK based systems when having the normalised Doppler frequency of $f_{d}=0.03$.

2) Performance of IrCC-URC-DMPSK coding scheme: The numerical results of Fig. 20 also show that for a sufficiently high number of say $J \geq 30$ outer iterations, the distance between the capacity curve of the IrCC-URC-MSDD-aidedDQPSK scheme and that of the DCMC-MSDD-aided-DQPSK arrangement is less than $0.5 \mathrm{~dB}$.

Setting IrCC coding coefficients as seen in Fig. 19, as well as the number of inner iterations to $I=2$, and the number of outer iterations to $J=1,10,20,25,30$, we can see the relationship between the performance of the coding scheme and its complexity, where the complexity is represented by the number $J$ of iterations. This relationship can also be observed on Fig. 20, where again the capacity of the system is seen to vary along with the number $J$ of outer iterations. This suggests that the area property of the EXIT chart can be beneficially used for determining the most appropriate number of iterations between a pair concatenated coding components.

\section{Benefit of sub-frame transmission}

The EXIT chart-aided design procedure has been shown to be an effective tool of designing irregular coding schemes that are capable of approaching the fast-fading channel's capacity. More explicitly, the fast-fading channel's capacity may be determined by the versatile tool of EXIT charts, as detailed in Section III-A, Section III-B and Section III-C. When slow fading is encountered, the concept of outage probabilities detailed in Section IV has to be used for providing a benchmarker for the coding schemes under study. In [127]-[132], the sub-frame based transmission mode has been invoked in slow-fading environments for the sake of improving the system's performance, which is hence expected to be as good as that exhibited in fast-fading environments. To conclude our related discussion, Section V-D aims for accentuating the benefit of employing the sub-frame based transmission mode as well as the advantages of using the outage probability as a benchmarker for near-capacity coding schemes.

In the fast (small-scale) fading channel, the transmitter can send data at the rate of $R<\left.C\right|_{h}$, while maintaining an arbitrarily small error probability, but this idealised performance cannot be maintained for block fading channel [116]. Let us take one of the near-capacity coding schemes, for example the IrCC-URC-MSDD-aided-DQPSK scheme designed in previous sections to illustrate the scenario. As above-mentioned, in the practical system [127]-[130], each channel-encoded frame tends to be transmitted in an $N_{s u b}$ sub-frames, where the average $S N R$ value at the receiver side may be formulated as:

$$
S N R_{r}=\frac{\mathrm{E}\left[\left|h_{s}\right|^{2}\right]}{N_{0}}=\frac{\sum_{i=1}^{N_{s u b}}\left|h_{s, i}\right|^{2} / N_{s u b}}{N_{0}},
$$

with $h_{s, i}$ representing the block shadow fading corresponding to the $i^{t h}$ sub-frame of the channel-encoded frame.

It is expected that by partitioning each encoded frame into subframes [131], [132], the diversity order of the transmission link is increased, thereby improving the attainable performance of the transmission link for transmission over the block fading channel. As a result, the detrimental influence of a single deep fade spreads over a larger number of encoded frames. Hence, the propagation effects of a fast fading scenario can be mimicked.

Let us continue by considering the performance of the IrCC-URC-MSDD-aided-DBPSK coding scheme for pointing out the specific benefit of employing of subframes. More specifically, it can be seen in Fig. 21 that while the number $N_{s u b}$ of the sub-frames is increasing from $N_{s u b}=1$ to $N_{s u b}=$ $10^{4}$, the performance of the IrCC-URC-MSDD-aided-DBPSK scheme recorded for transmission over a channel, where both the block shadow fading and the small-scale Rayleigh fading are taken into consideration, approaches that of the IrCC-URCMSDD-aided-DBPSK scheme communicating over the smallscale Rayleigh fading channel.

In brief, the employment of the sub-frame based transmission can be interpreted as follows. A near-capacity channel 


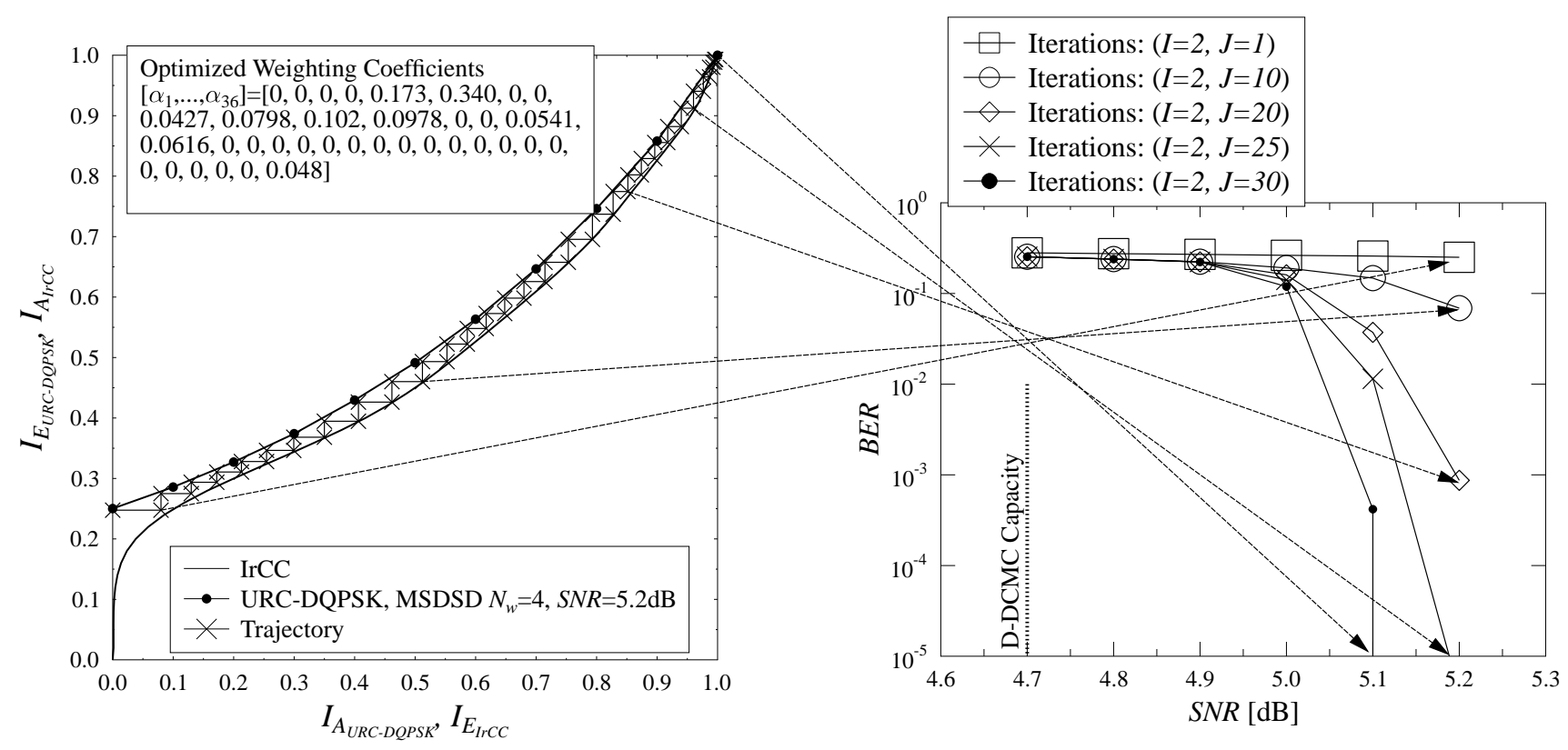

Fig. 19. The EXIT curves and performance of the IrCC-URC-MSDD-aided-DQPSK scheme.

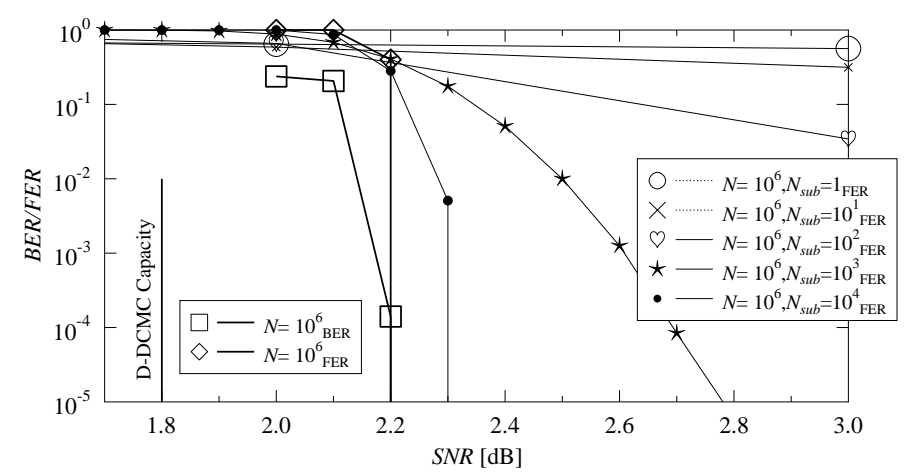

Fig. 21. Performance of the IrCC-URC-MSDD-aided-DBPSK scheme in the small-scale Rayleigh fading channel $\left(N=10_{B E R}^{6}\right.$ and $\left.N=10_{F E R}^{6}\right)$ with the normalised Doppler frequency $f_{d}=0.03$ as well as in the block fading channel, the number of sub-frames ranging from $N_{s u b}=1$ to $N_{s u b}=10^{4}$ for allocating an encoded frame of $N=10^{6}$ bits.

coding scheme can be designed for the sake of providing the time diversity within a frame. Hence an arbitrarily low BER may be achieved in a small-scale fading environment, where the channel conditions vary on a symbol-by-symbol basis. For the block fading channel, where the channel conditions within a frame duration are determined by a single channel coefficient, inter-frame diversity provided by the sub-frame based transmission regime may be exploited for attaining a good performance.

\section{E. Non-Coherent versus Coherent Near-Capacity Schemes}

Having presented philosophy of the near-capacity designs for both the coherent detection schemes of Section V-B and for the non-coherent detection schemes of Section V-C may pose to a dilemma, namely as to whether the coherent or non-coherent system would be favoured in a given scenario. In order to discuss this dilemma, let us now provide further insights into the effects of channel estimation errors on the performance of coherent systems. The influence of channel estimation in coherent detection based schemes can be characterised by the term $\theta$ defined as the normalised channel estimation accuracy, which may be formulated as:

$$
\theta=100 \times \mathrm{E}\left[1-\frac{|| h|-| \hat{h}||}{|h|}\right],
$$

where $\hat{h}$ is the estimated value of the channel coefficient $h$. It should be noted that if we have $\frac{|| h|-| \hat{h}||}{|h|}>1$, the accuracy is equal to zero, $\theta=0$. For the sake of readability, the estimated channel coefficient of $\hat{h}$ is deemed to be formed by

$$
\hat{h}=h+n_{\text {est }},
$$

where $n_{\text {est }}$ is likened to the estimated noise springing from the inaccuracy of the channel estimator. The estimated noise of $n_{\text {est }}$ can be further considered as an AWGN process having a variance of $N_{0, e s t} / 2$ per dimension at a nominal estimated signal to noise ratio of $S N R_{\text {est }}$. Accordingly, as seen in Fig. 22, the normalised channel estimation accuracy of $\theta$ given in (14) varies according to different frame lengths $N$ ranging from $N=10^{1}$ bits to $N=10^{6}$ bits, as plotted against different values of $S N R_{\text {est }}$ listed in Tab. VII.

The benefit of introducing the estimated signal to noise ratio of $S N R_{\text {est }}$ is that we can examine the performance's degradation of the coherent system employing a practical channel estimator, compared to the performance of the coherent system assuming that the channel state information is available at the receiver.

It can be inferred from Fig. 22 that the value of $S N R_{\text {est }}$ corresponding to a given value of the channel estimation accuracy can be calculated. For example, Table VII lists the values of $S N R_{\text {est }}$ associating with different values of normalised channel estimation accuracy, namely from $10 \%$ to $100 \%$. 


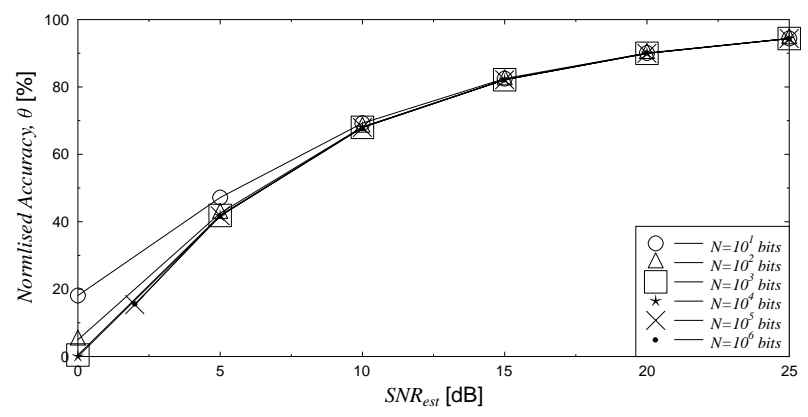

Fig. 22. The normalised channel estimation accuracy of $\theta$, when considering different frame lengths $N$ ranging from $N=10^{1}$ bits to $N=10^{6}$ bits.

\begin{tabular}{|c|c|}
\hline Normalised channel estimation accuracy, $\theta[\%]$ & $S N R_{\text {est }}[\mathbf{d B}]$ \\
\hline $10 \%$ & 1.676 \\
\hline $20 \%$ & 2.518 \\
\hline $30 \%$ & 3.554 \\
\hline $40 \%$ & 4.783 \\
\hline $50 \%$ & 6.276 \\
\hline $60 \%$ & 8.127 \\
\hline $70 \%$ & 10.571 \\
\hline $80 \%$ & 14.027 \\
\hline $90 \%$ & 20.015 \\
\hline $100 \%$ & 100.667 \\
\hline
\end{tabular}

TABLE VII

$S N R_{\text {est }}$ VALUES CORRESPONDING TO DIFFERENT VALUES OF THE NORMALISED CHANNEL ESTIMATION ACCURACY $\theta$ RANGING FROM $\theta=10 \%$ TO $\theta=100 \%$, WHEN APPLYING THE FRAME LENGTH $N=10^{6}$ BITS.

Employing the channel estimation noise conventionally corresponding to $S N R_{\text {est }}$ given by Table VII, we can evaluate the performance of the coherent coding schemes, for example IrCC-URC-QPSK scheme, when different levels of the channel estimation accuracy are achieved by the channel estimator. By doing comparison between the performance of the coherent scheme and that of the non-coherent counterpart, the threshold value of the channel estimation accuracy can be determined.

For example, the performance comparison of the IrCCURC-QPSK scheme in different regimes, namely both in the non-coherent and in the coherent regime was carried out by taking into consideration a realistic channel estimator. As seen in Fig. 23, the performance of the system employing IrCCURC-DQPSK coding scheme is equivalent to that of IrCCURC-QPSK, provided that of the channel estimator is capable of achieving an accuracy of $\theta=60 \%$. As a result, the threshold of $\theta=60 \%$ can be used by the system to switch between coherent and non-coherent detection regime.

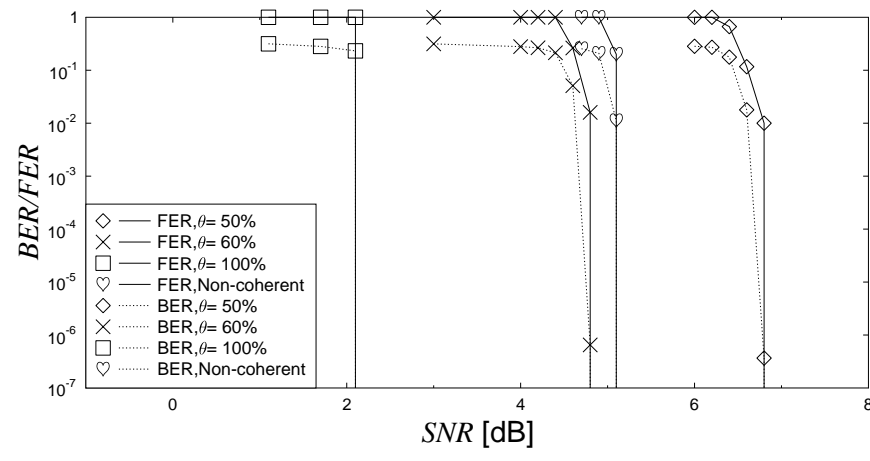

Fig. 23. Comparison of the non-coherent versus coherent: IrCC-URC-QPSK scheme versus IrCC-URC-MSDD-aided-DQPSK, the frame length of $N=$ $10^{5}$, when communicating over the small-scale Rayleigh fading channel.

\section{ApPliCATION In COOPERATIVE COMMUNiCATION}

Classically, relays have been used for extending the range of wireless communication systems [23], [133]-[138]. However, in recent years, numerous exciting new applications of relay aided communications have emerged [139]-[141], including the design of efficient cooperative protocols and of distributed signal processing techniques [142]. By designing sophisticated cooperative medium access protocols for the source and relay nodes [143], [144] in conjunction with appropriate modulation and coding [145], [146], it has been found that the diversity gain of the system can be substantially improved [147]. The cooperative protocols may be categorised depending on the specific operations [117], [148], [149] carried out at the relays, namely as amplify-and-forward (AAF), demodulation and forward (DemAF), decode and forward (DAF) as well as adaptive relaying protocol (AFP).

Distributed signal processing techniques cast in the context of multi-component coding schemes employing relay-aided transmissions focus on the joint signal and coding design at both the source, as well as at the relay and destination nodes. Such a joint coding design is referred to as distributed coding. Naturally, having the distributed nature constitutes the crucial difference between the distributed coding scheme and its conventional counterpart. The distributed nature manifests itself in terms of constructing of encoded codewords at the destination node, which are transmitted via different propagation paths. Having this distributed construction lends a higher degree of freedom to the system, which is achieved at the cost of a more complex design and implementation. The distributed coding schemes may be classified into five categories, namely distributed source-channel coding schemes [150]-[152], distributed network and channel coding [153], [154], distributed low density parity check codes (LDPC) [155], [156], distributed turbo coding schemes [157]-[159] and finally distributed space time coding [45], [160]. In all the above-mentioned distributed schemes [45], [151], [154], [156], [159], EXIT charts were shown to be an effective tool used for designing the distributed schemes for approaching the corresponding channel capacity.

In order to demonstrate the benefits of EXIT charts in designing distributed coding schemes, we will illustrate the code design principles presented in Section V-A with the aid of a typical example, where distributed turbo and spacetime coding schemes were designed jointly. Let us commence by describing an example of the typical cooperative systems presented in Fig. 24, in which a source node $(S)$ and a destination node $(D)$ are communicating through $N_{R}=2$ relay nodes $(R)$ due to the low quality of the signal transmitted from the $S$ node at the $D$ node. Hence the SD link is often assumed to be unavailable [161]. For the case of considering the availability of the signal transmitted via the SD link, please allow us to refer interested readers to Ref. [45], [159]. Note that the number of relay nodes $N_{R}=2$ is chosen for clarity of presentation. A transmission session of the system detailed in Fig. 24 is accomplished in two transmission periods. The first transmission period is for the transmission from the $S$ node to the $R$ nodes, while the other is for the transmission from 
the $R$ nodes to the $D$ node.

\section{A. Distributed System Model}

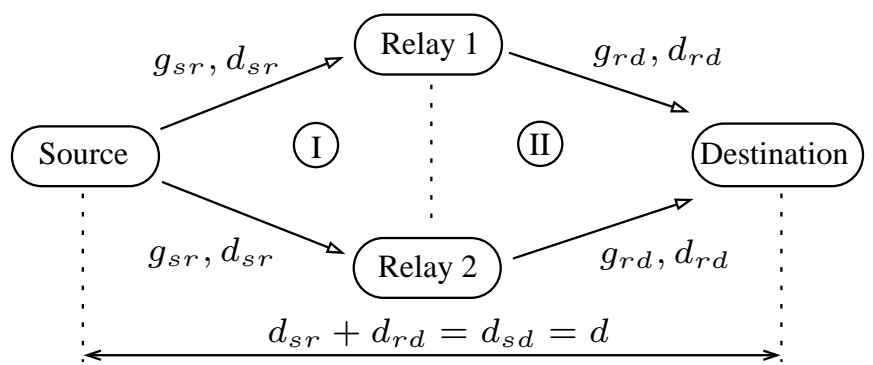

Fig. 24. The model of the distributed coding system in the absence of the source-destination link.

Typically, during the first transmission period, the $S$ node transmits its coded frame to the $R$ nodes. Then, after decoding and re-encoding the signals received from the $S$ node, the two $R$ nodes transmit the resultant coded frame to the $D$ node during the second transmission period. At the destination, the signals received from the two single-antenna aided relays are combined, detected and decoded, in order to recover the information transmitted from the $S$ node. Although the signals are transmitted from two separate $R$ nodes having a single antenna, these signals are treated, as if they came from a single relay having two transmit antennas. Naturally, the two relays are required to collaborate, which is further elaborated on Section VI-B, where the structure of the distributed encoder and decoder is described.

As seen on the system model presented in Fig. 24, owing to the advantageous position, the relays have a benefit of the power-gain (or geometrical pathloss reduction) w.r.t. the source-to-relay and relay-to-destination links, namely $g_{s r}$ and $g_{r d}$. The magnitude of the power-gain is proportional to the ratio of the two respective distances. Naturally, the power-gain of the source-to-destination link with respect to itself is unity, i.e. $g_{s d}=1$. For the sake of simplicity, we may stipulate another assumption, namely that the $R$ nodes are located on the straight line from the $S$ node to the $D$ node. For the convenience of presentation, the unconventional $S N R_{t}$ [14], [45], [162] was defined as the ratio of the power transmitted from the transmitter to the noise power encountered at the receiver ${ }^{1}$. Accordingly, we have the ratio of the received power to the noise power at the receiver

$$
S N R_{r}=G+S N R_{t},
$$

where $G(\mathrm{~dB})$ is the power gain of the link.

\section{B. Structure of Distributed Turbo Space-Time Coding}

As above-mentioned, cooperative communication has been shown to be an effective technique of allowing single antenna aided users to share their antennas to create a virtual MIMO system, thus providing extra spatial diversity in wireless networks. Distributed turbo and space-time coding schemes [45],

\footnotetext{
${ }^{1}$ This definition is unconventional, because it relates physical quantities measured at different locations. However, it is convenient for illustrating the benefit the geometrical pathloss reduction.
}

[157]-[160] are inherently capable of supporting VMIMO systems with the goal of facilitating near-capacity operation. An example of the distributed turbo coding scheme used for the system model portrayed in Fig. 24 is presented in Fig. 25. In the DC-IrCC-URC-STTC coding scheme depicted in Fig. 25, a two-stage encoder constituted by an Irregular Convolutional Code and Unity-Rate Code(IrCC-URC) is employed at the $S$ node. By contrast, at both the two $R$ nodes and $D$ node, the three-stage coding scheme constituted by the IrCC-URCSTTC is employed. It should be noted that the collaboration of the two $R$ nodes has to guarantee that at any instant only the signals of a single STTC component of the single-antenna $R$ nodes is transmitted. Thus, the signals received at the $D$ node are a combination of the signals received from the two relays during the second transmission period. Then, the signals received at the $D$ node are treated, as if they arrived from a single relay transmitter equipped with two co-located transmit antennas. In the illustrative example, 8PSK modulation is employed for the two-stage IrCC-URC coding scheme of the $S$ node, while only QPSK modulation is used for the threestage IrCC-URC-STTC.

In the model of the distributed coding scheme depicted in Fig. 25, the information bit sequence $u$ is encoded by the IrCC encoder at the $S$ node for producing the coded sequence $c$, before passing $c$ through the interleaver $\pi_{1}$. The interleaved bit sequence is encoded by the URC encoder and then modulated using 8PSK or QPSK, in order to form the transmitted symbol sequence $x$. The signals $y_{r 1}$ and $y_{r 2}$ received at the both relays are iteratively decoded by the IrCC-URC decoders, as seen in Fig. 25, in order to estimate the original information bit sequences, $u_{1}$ and $u_{2}$, at $R$ node 1 and 2 , respectively. Then the estimated bit sequences of $u_{1}$ and $u_{2}$ are passed through the IrCC-URC-STTC encoding process relying on the pair of interleavers, $\pi_{1}$ and $\pi_{2}$, which are located between the IrCC encoder and URC encoder as well as between the URC encoder and the STTC encoder, respectively. These two interleavers facilitate the provision of hitherto in exploited extrinsic information for the iterative decoding process at the destination, where the iterations exchanging extrinsic information between the STTC decoder and the URC decoder are performed $I$ times, before one of $J$ times iteration between the URC decoder and the IrCC decoder is carried out, as detailed in Section VI-C. In other words, once $I$ inner iterations are accomplished, one iteration of $J$ outer iterations is triggered before passing the information back to the inner iterations. This process is repeated, until all the affordable number of $J$ outer iterations are performed, as seen in the destination block of Fig. 25.

Then, QPSK modulation is employed at the output of the $R$ node's STTC encoder. Note that the sequences transmitted by each of the relays, namely $x_{r 1}$ and $x_{r 2}$, are created by choosing only one predefined output of the two-antenna 4state STTC encoder of [11]. The sequence received at the $D$ node, namely $y_{d}$ seen in Fig. 25, is then subjected to the inverse process: $y_{d}$ is demodulated, and finally the resultant sequence is decoded by two iterative decoders, namely by the URC-STTC decoder and the IrCC-[amalgamated-URC-STTC] decoder of Fig. 25. The decoded information bit sequence $u^{\prime}$ 


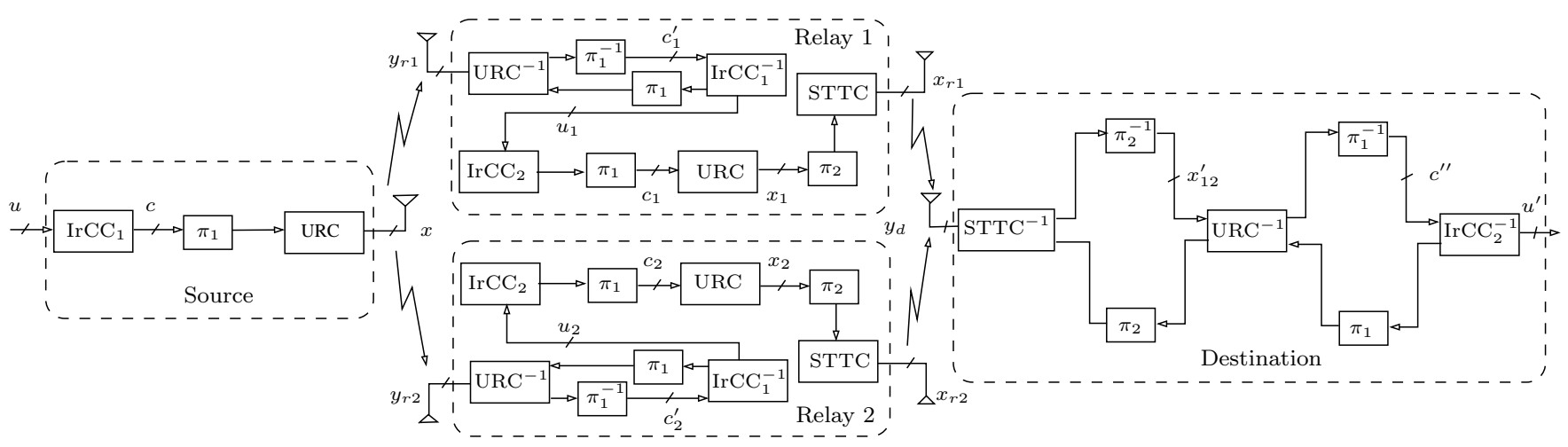

Fig. 25. The block diagram of the Distributed Concatenated Irregular Convolutional Code, Unity-Rate Code and Space-Time Trellis Code scheme for the system model presented in Fig. 24. This scheme relies on the IrCC-URC-STTC-MPSK arrangement detailed in Section VI-C and on the Irregular Convolutional Coded Unity Rate Coded M-ary Phase Shift Keying (IrCC-URC-MPSK) arrangement presented in Section V-B.

seen in Fig. 25 is compared to the original one for evaluating the performance of the system.

The URC, IrCC and STTC component codes have the code rates of $R_{U R C}, R_{I r C C}$ and $R_{S T T C}$, respectively, where $R_{S T T C}=1$ in the example.

Accordingly, the system's overall throughput of $\eta$ representing the relationship between the $E_{b} / N_{0}$ and $S N R$ values of the system can be formulated as:

$$
\eta=\frac{N_{i}}{N_{s}+N_{r}}=0.6[B P S],
$$

where $N_{i}$ is the number of information bits transmitted within a duration of $\left(N_{s}+N_{r}\right)$ symbol periods, while $N_{s}$ and $N_{r}$ are the number of symbols transmitted by the $S$ node and the $R$ node within a transmission session, respectively. It should be noted that the system's overall throughput $\eta$ also denotes the average number of bits conveyed by a symbol transmitted in the distributed system. Hence, when comparing the distributed system to the single link transmission system, the system's overall throughput and the number of modulated bits defined in Section III-B are equivalent.

\section{Design of distributed IrCC-URC-STTC-MPSK coding scheme}

The design of the distributed coding scheme portrayed in Fig. 24 relies on the IrCC-URC-MPSK scheme presented in Section V-B and on the IrCC-URC-STTC regime portrayed in Fig. 26. Going beyond the design procedure presented in Section V-B and Section V-C, the tool of EXIT charts may also be used for optimising the BER performance of the entire distributed coding scheme, so that it becomes capable of approaching the corresponding cooperative channel capacity. In order to design such distributed coding schemes, we can use EXIT charts for finding the most appropriate configurations of the entire coding scheme. Hence the coding scheme becomes capable of attaining the best possible performance, provided that the position of relays and their transmit powers are given. Alternatively, we can pursue an adaptive approach, where a set of the most appropriate system configurations is determined by employing EXIT charts. Then the system will adaptively adjust its various configuration to adapt to the specific relay positions and transmit powers available.

For the ease of presentation, the time-invariant approach is used here for portraying the design of the IrCC-URC-STTCMPSK coding arrangement detailed in Fig. 26. According to the structure of the IrCC-URC-STTC scheme featured in Fig. 26, the bit stream at the transmitter side is first encoded by the IrCC encoder before it is fed into the interleaver $\pi_{1}$, in order to get the interleaved input stream for feeding to the URC encoder. The output of the URC encoder is again scrambled by the interleaver $\pi_{2}$ before being encoded by the STTC detailed in Section II-C. This signal is then transmitted to the receiver side over wireless fading channels, as portrayed in Fig. 26.

At the receiver side, as seen in Fig. 26, the signals provided by the receive antenna are demodulated and decoded by the STTC decoder before being processed by the $I$ inner iterations exchanging extrinsic information between the STTC decoder and the URC decoder. The resultant soft information extracted from the received signals by the inner iterations are then used as the input data for the $J$ outer iterations exchanging extrinsic information between the $\mathrm{IrCC}$ encoder and the amalgamated inner component, which is the Unity Rate Coded M-ary Phase Shift Keying (URC-STTC-MPSK) arrangement. Hence, the design goals are to determine the most appropriate number of inner and outer iterations, as well as the weighting coefficients of the IrCC subcodes used in the IrCC codec.

Note that in the distributed coding scheme portrayed in Fig. 25, the IrCC-URC-STTC-MPSK coding scheme is invoked for constructing a virtual Multi-Input MultiOutput (MIMO) scheme constituted by the relay-aided system. In the following, we will show how the EXIT-chart based design principles outlined in Section V-A can be applied for designing the specific IrCC-URC-STTC coding scheme relying on MPSK modulation. Accordingly, we will present design results associated with the IrCC-URC-STTC-QPSK arrangement employing 17-subcode $\mathrm{IrCC}$ as a numerical example.

1) EXIT-chart matching and optimisation of the IrCC-URCSTTC-MPSK scheme: Firstly, the two-stage inner arrangement URC-STTC-QPSK is designed. Secondly, the design process 

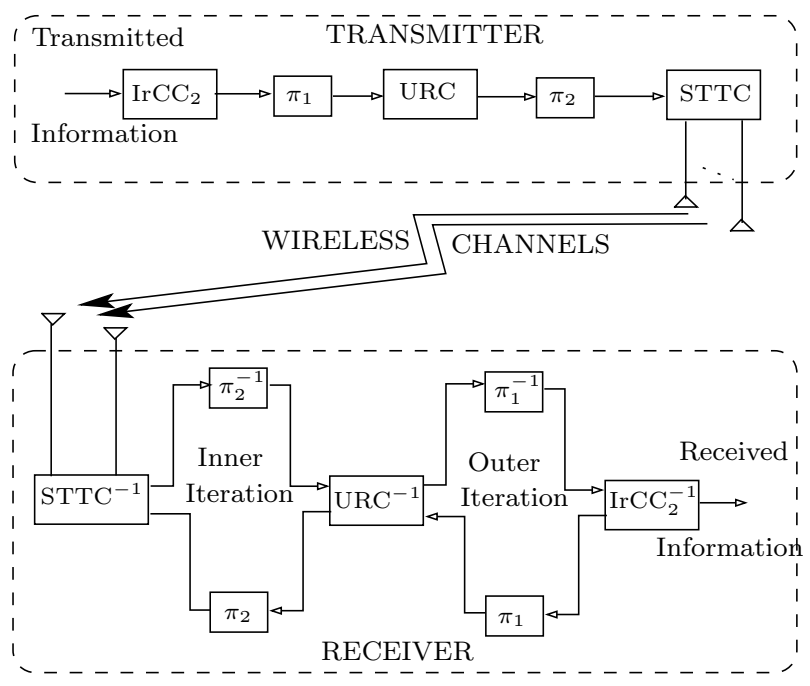

Fig. 26. The structure of the Irregular Convolutional Coded Unity Rate Coded Space Time Trellis Code (IrCC-URC-STTC) coding scheme.

is continued by viewing the three-stage IrCC-URC-STTCQPSK coding arrangement as the two-stage-concatenated IrCC outer code and the amalgamated URC-STTC-QPSK inner code. Again the design guidelines introduced in Section V-A can be applied for both the two coding arrangements.

For the two-stage inner Unity Rate Coded Space Time Trellis Code Quadrature Shift Keying (URC-STTC-QPSK) arrangement, the design task can be interpreted as that of increasing the achievable channel capacity of the amalgamated URC-STTC-QPSK inner code, in order to approach that of a Space Time Trellis Coded (STTC) and STTC-QPSK aided system. Accordingly, the area property of EXIT charts can be exploited for calculating the DCMC capacity of the two inner-most coding arrangements, namely that of the Space Time Trellis Code Quadrature Shift Keying (STTC-QPSK) and URC-STTC-QPSK schemes. Then, based on the capacity of these two coding arrangements, the most appropriate number of iterations $I=3$ is determined. As shown Fig. 27 shows that once at least $I=3$ iterations had been applied, the achievable channel capacities of the STTC-QPSK and URC-STTC-QPSK systems become near-identical.

For the three-stage IrCC-URC-STTC-QPSK coding arrangement, firstly the EXIT chart of the URC-STTC-QPSK scheme is created for different receiver signal to noise ratios $S N R_{r}$. Secondly, EXIT-curve the matching algorithm is applied for the IrCC codes having a total rate of $R_{c}=0.5$ for example, in order to determine the optimised weighting coefficients $\alpha_{j}, j=1, \ldots, 17$, of the 17 -subcode IrCC codes corresponding to the lowest possible $S N R_{r}$. As a result, the EXIT-chart results corresponding to the optimised weighting coefficients reveal the optimal number of iterations between the IrCC and URC-STTC-QPSK block. This optimal number of iterations was found to be $J=24$ in Fig. 27.

Again, by exploiting the area property of the EXIT-charts detailed in Section II-A2, the achievable DCMC capacities of the $\mathrm{MIMO}_{2 \times 1}$-QPSK, the $\mathrm{STTC}_{2} \times 1$-QPSK, URC$\mathrm{STTC}_{2} \times 1$-QPSK and IrCC-URC-STTC $2 \times 1$-QPSK aided systems are quantified for determining the associated capacity curves, as plotted in Fig. 27. It should be noted that the capacity of an inner arrangement sets an upper bound for the capacity of an outer arrangement. Hence, according to the afore-listed order, the capacity associated with the inner most arrangement $\mathrm{MIMO}_{2 \times 1}$-QPSK sets the maximum achievable capacity for all the systems employing the other schemes, namely the $\mathrm{STTC}_{2} \times 1$-QPSK, URC-STTC ${ }_{2} \times 1$-QPSK and IrCC-URC-STTC $\mathrm{C}_{2} \times 1$-QPSK, as seen in Fig. 27.

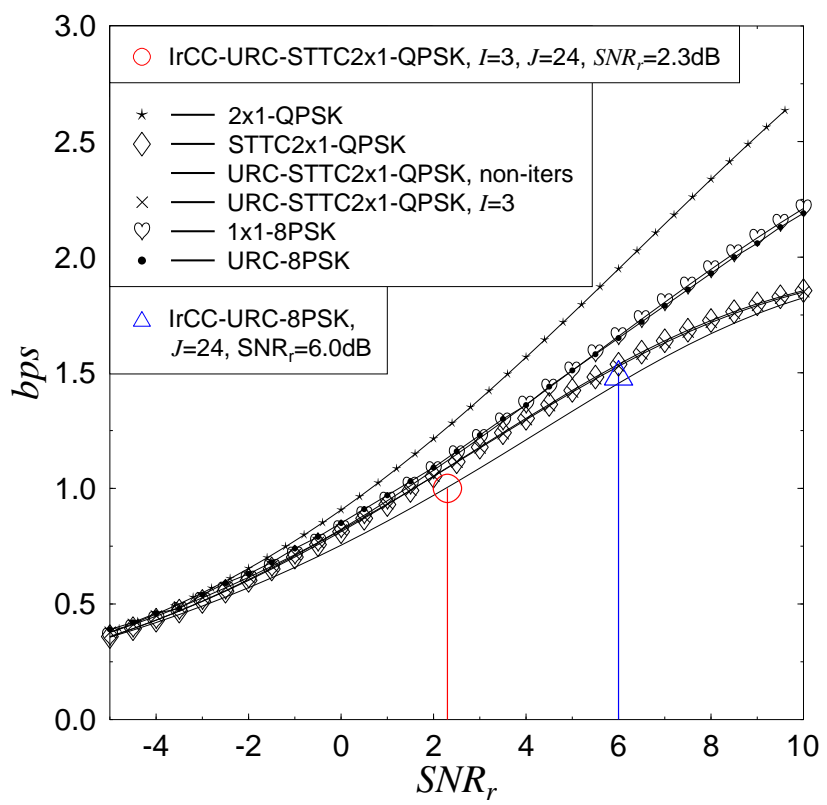

Fig. 27. Channel capacity comparison for the IrCC-URC-STTC2x1-QPSK and IrCC-URC-8PSK systems.

Following the same procedure as illustrated by designing the IrCC-URC-STTC-QPSK, other coding arrangements relying on 8PSK and 16PSK, namely the IrCC-URC-STTC-8PSK and IrCC-URC-STTC-16PSK schemes were also designed. As a result, the corresponding weighting coefficients of the IrCC encoder are listed in Table VIII.

\begin{tabular}{|l|l|}
\hline Arrangement (Turbo-cliff $S N R$ ) & \multicolumn{1}{|c|}{ Coefficients: $\left[\alpha_{1}, \alpha_{2}, \ldots, \alpha_{17}\right]$} \\
\hline URC-STTC-QPSK $(2.3 \mathrm{~dB})$ & {$[0.05,0,0,00.169,0.219,0.036$,} \\
& $0.023,0.0166,0.149,0.015$, \\
& $0.089,0.058,0,0.093$, \\
& $0.033,0.044]$ \\
\hline URC-STTC-8PSK $(4.4 \mathrm{~dB})$ & {$[00.171,0.093,0,0,0.195,0,0$,} \\
& $0.099,0.05,0,0,0.197$, \\
& $0,0,0.025,0.165]$ \\
\hline URC-STTC-16PSK (7.0 dB) & {$[0.203,0,0.093,00.102,0,0$,} \\
& $0.148,0,0,00.055,0.149$, \\
& $0,0,0,0.248]$ \\
\hline
\end{tabular}

TABLE VIII

SUBCODE WEIGHTING COEFFICIENTS OF THE IRCC ENCODER ASSOCIATED WITH URC-STTC-QPSK, URC-STTC-8PSK AND URC-STTC-16PSK.

Importantly, the value of the 'turbo-cliff' SNR given in Table VIII indicates that once the SNR value exceeds this value, the BER/FER of the coding scheme is expected to become 
infinitesimally low. Indeed, the performance prediction provided by the EXIT-chart curves, which were created by using the procedure briefly summarised in Section V-A, is always satisfied by Monte Carlo simulation results. Accordingly, the BER of the system supported by the coding schemes, such as the IrCC-URC-STTC-QPSK, IrCC-URC-STTC-8PSK and IrCC-URC-STTC-16PSK arrangements, drops to a value below $10^{-6}$, when the SNR value exceeds the corresponding turbo-cliff SNR.

\section{EXIT chart analysis in relay selection}

As mentioned above, EXIT charts can also be employed more widely than that demonstrated in Section V-B and Section V-C. A further application is that of optimising the BER/FER performance of the entire distributed coding scheme upon activating the appropriate relays at a favourable position or upon assigning the most appropriate transmit power to the relays for the sake of approaching the corresponding cooperative channel capacity. In what follow, we will exemplify the further employment of EXIT charts in the context of the distributed coding scheme featured in Fig. 25.

In order to allow the distributed coding scheme detailed in Fig. 25 to attain the best possible performance, a pair of appropriate relays should be utilised. Without any loss of generality, it can be assumed that a sufficiently high number of relays are roaming between the source and destination. For the sake of employing appropriate active relays, one can either choose two relays at an appropriate position or equivalently choose two relays at an arbitrary position, provided that accurately controlled transmit powers are used both at the $S$ node and at the two $R$ nodes. These two strategies have the same influence on the system, since they result in the same received power.

For illustrating the further application of EXIT chart analysis to the relay selection issues, let us consider the first strategy. Accordingly, There might be two approaches for choosing the activated ones from the set of available relays. The first approach of selecting the relays located at the halfway position is straightforward. By contrast, the second approach relies on a more sophisticated idea, namely on ensuring that the appropriately shaped EXIT tunnel of the system remains open:

1) On the first approach, the system should activate two arbitrary relays that are about half-way between the $S$ node and the $D$ node. This approach can be generalised by picking two arbitrary relays that are close to each other and they both have a similar distance from both the $S$ node and $D$ node.

2) The more sophisticated EXIT-chart based approach appoints the optimal relays, which are those that facilitate the creation of an open EXIT tunnel, as presented in Section VI-C1 and Section V-B1, leading to the $(1,1)$ point at sufficiently high $S N R_{r}$ values at both the $R$ node and $D$ node, provided that the $S N R_{t}$ value is also sufficiently high. More specifically, the EXIT charts can be used for determining $S N R$ values corresponding to the BER turbo-cliffs of the two respective coding arrangements, namely those of the IrCC-URC and the
IrCC-URC-STTC arrangements. Upon the difference $a$ of those $S N R$ values, the optimal relay-position conditions are established as follows [42]:

$$
\begin{gathered}
d_{s r}=\frac{d}{(1+\sqrt{a})}, d_{r d}=\frac{d \times \sqrt{a}}{(1+\sqrt{a})}, \\
g_{s r}=(1+\sqrt{a})^{2}, g_{r d}=\frac{(1+\sqrt{a})^{2}}{a} .
\end{gathered}
$$

For example, let us consider the numerical results pertaining to the coding scheme detailed in Fig. 25, where 8PSK is used for the source-relay transmission, while QPSK is employed for the transmission between the $R$ nodes and $D$ node. The URC, IrCC and STTC components have the code rates of $R_{U R C}=1.0, R_{I r C C}=0.5$ and $R_{S T T C}=1.0$, respectively. Accordingly, one can find the value of $A=10 \log _{10} a=$ $3.7 \mathrm{~dB}$ by comparing the two $S N R$ values representing the respective open EXIT tunnels, as given in Tab. IX. By substituting the values of $A$ into Eq. (18), the optimal position of the relays given in Tab. $\mathrm{X}$ can be determined.

\begin{tabular}{|l|c|}
\hline \multicolumn{1}{|c|}{ Coding Schemes } & Convergent Point, $S N R_{r}[\mathbf{d B}]$ \\
\hline \hline IrCC-URC-STTC-QPSK & 2.3 \\
\hline IrCC-URC-8PSK & 6.0 \\
\hline
\end{tabular}

TABLE IX

CONVERGENCE-SNRS EXTRACTED FROM EXIT CHARTS, NAMELY OF THE IRCC-URC-8PSK SCHEME LISTED IN TAB. VI AND OF THE IRCC-URC-STTC-QPSK SCHEME LISTED IN TAB. VIII. THESE EXIT CHARTS WERE OBTAINED IN A RAYLEIGH SMALL-SCALE FADING SCENARIO.

\section{E. Relay Positioning and its Power Gain}

In this section, we discuss the achievable BER versus $E_{b} / N_{0}$ performance in comparison to the associated attainable cooperative channel capacity based on the two different relayselection approaches, in order to highlight the employment of the EXIT-chart aided design tool.

The cooperative channel capacity can be calculated by applying the general formulae given in [17] in the context of the specific example detailed in this paper. Accordingly, the components of the specific formula need to be estimated by exploiting the area property of the EXIT chart detailed in Section II-A2. Further details elaborating on the calculation of the cooperative channel capacity are presented in [45], [49].

Let us now consider the BER-performance of the distributed coding scheme relying on the IrCC-URC-STTC regime from different perspectives. Although perfect relaying is not required for the success of the coding scheme, benchmark results for the perfect-relaying scenario, where no errors are imposed by the source-relay link, are presented for showing the achievable upper bound performance. Note that the term 'practical relay' indicates that the quality of the source-torelay transmission link reflects the performance of the channel coding scheme used. Accordingly, let us consider the four configurations of the system portrayed in Fig. 25. These configurations listed in Tab. XI are different in terms of the position and type of relays activated as well as in the 


\begin{tabular}{|c|c|c|c|}
\hline \multicolumn{2}{|c|}{$\begin{array}{c}\text { Distributed Coding Scheme } \\
\text { Relay-Destination }\end{array}$} & $\begin{array}{c}A \text { (Convergence } \\
\text { SNR Difference) }\end{array}$ & Optimal Position \\
\hline \hline IrCC-URC-QPSK & IrCC-URC-STTC-QPSK & 0.5 & $d_{s r}=0.48 d, d_{r d}=0.52 d$ \\
\hline IrCC-URC-8PSK & IrCC-URC-STTC-QPSK & 3.7 & $d_{s r}=0.39 d, d_{r d}=0.61 d$ \\
\hline
\end{tabular}

TABLE X

OPTIMAL POSITIONS OF THE RELAYS.

\begin{tabular}{|c|c|c|c|}
\hline Configuration & Position of relays & Type of relays & \multicolumn{1}{c|}{ Scheme in use } \\
\hline \hline 1 & Half way & Perfect & $S-->R:$ IrCC-URC-8PSK \\
& & $R-->D:$ IrCC-URC-STTC-QPSK \\
\hline 2 & Half way & Practical & $S-->R:$ IrCC-URC-8PSK \\
& & $R-->D:$ IrCC-URC-STTC-QPSK \\
\hline 3 & Optimal & Perfect & $S-->R:$ IrCC-URC-8PSK \\
& & $R-->D:$ IrCC-URC-STTC-QPSK \\
\hline 4 & Optimal & Practical & $S-->R:$ IrCC-URC-8PSK \\
& & $R-->D:$ IrCC-URC-STTC-QPSK \\
\hline
\end{tabular}

TABLE XI

CONFIGURATIONS OF THE SYSTEM PRESENTED IN FIG. 25, CORRESPONDING TO DIFFERENT MODULATION SCHEMES EMPLOYED FOR TRANSMISSION FROM THE $S$ NODE TO THE $R$ NODES, AS WELL AS THE POSITION AND TYPE OF THE RELAYS IN USE.

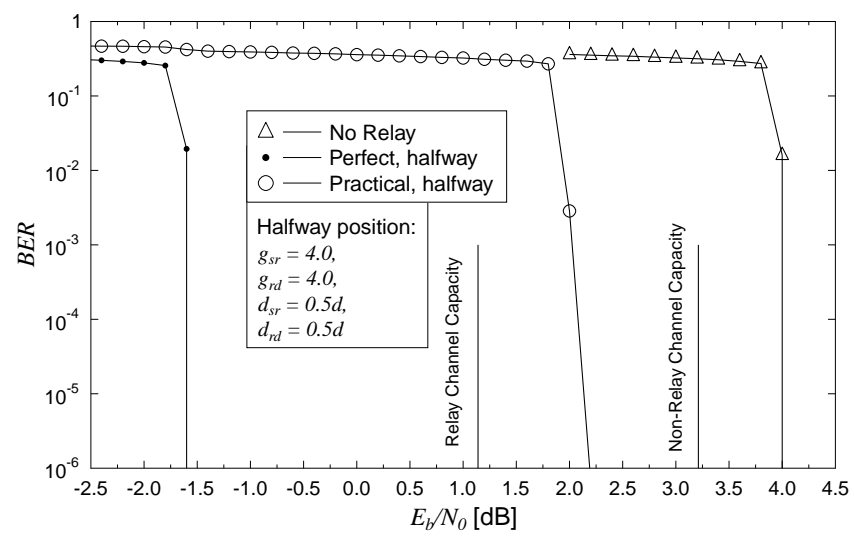

Fig. 28. BER-performance comparison of the distributed coding aided system of Fig. 25, when activating the $R$ nodes located half-way between the $S$ node and the $D$ node, which corresponds to the configuration 1 and 2 listed in Tab. XI, where the 8PSK modulation scheme is employed for the transmission link spanning from the $S$ node to the $R$ nodes. A Rayleigh-distributed smallscale fading scenario is considered for all the transmissions in the system.

modulation scheme used for the source-to-relay transmission links.

The performance of the distributed coding scheme recorded in Fig. 28 and Fig. 29 for all the four configurations clearly confirms the fact that having a distributed structure has system performance benefits. More specifically, as shown in Fig. 28, when having access to relays at the half-way position, an $E_{b} / N_{0}$ improvement of $2.0 \mathrm{~dB}$ is achieved at a BER of $10^{-5}$. The improvement becomes higher, for example the improvement of $4.0 \mathrm{~dB}$ at a $B E R$ of $10^{-5}$ seen in Fig. 29, when selecting the relays in the vicinity of the optimal position determined by the EXIT chart tool.

The performance gain of selecting relays at the optimum location, as opposed to stipulating the idealised simplifying assumption of having relays at the half-way location, will be higher when we have a higher value of $A$, which quantifies the difference between the pair of convergence $S N R_{r}$ values obtained by the EXIT chart analysis. In other words, EXIT chart analysis can be used for determining the proximity of the distributed coding scheme's performance to its optimal performance. The optimal performance can be achieved by adjusting the transmission rate of the coding components, by activating mobile relays that happen to be roaming in the vicinity of the optimal position or by accurately controlling the transmit power of both the source and of the relays nodes.

The performance of a practical relaying scheme, which may impose error-propagation owing to its decision-errors, was shown to be either about $0.2 \mathrm{~dB}$ or $3.6 \mathrm{~dB}$ from that of the idealised perfect relay scheme, which is due to the effects of error propagation imposed by the $R$ nodes, as seen in Fig. 28 . By definition, the optimal relay selection method aims for solving this problem by activating relays exactly at the optimal locations, so that the system may reach the $S N R_{r(\text { relay })}$ and $S N R_{r(\text { dest.) }}$ values required for simultaneously achieving a low BER at both the $R$ and $D$ nodes. The optimal relay nodes in the scheme considered are located at normalised positions of $0.39 d$ closer to the $S$ node, in order to achieve $g_{s r}=6.41$, $g_{r d}=2.73$. The size of the performance gap between the practical and the idealised perfect relaying depends on how close the distributed coding scheme operates to its optimal configuration, which can be ascertained with the aid of EXIT chart tool.

The distributed coding scheme is capable of operating at a similar SNR-distance, for example $1.1 \mathrm{~dB}$, from the relay realistic channel's capacity in case of both 'mid-way' and optimal relay positions. However, the channel capacity of these two scenarios is different due to the fact that the channel's capacity can be optimised by accurately tuning the distributed coding configuration to its optimum. Explicitly, this may be achieved by adjusting the coding rate of its coding components, by carefully activating relays roaming in the vicinity of the optimal position or by accurately controlling the transmit power of both the source and relays nodes. 


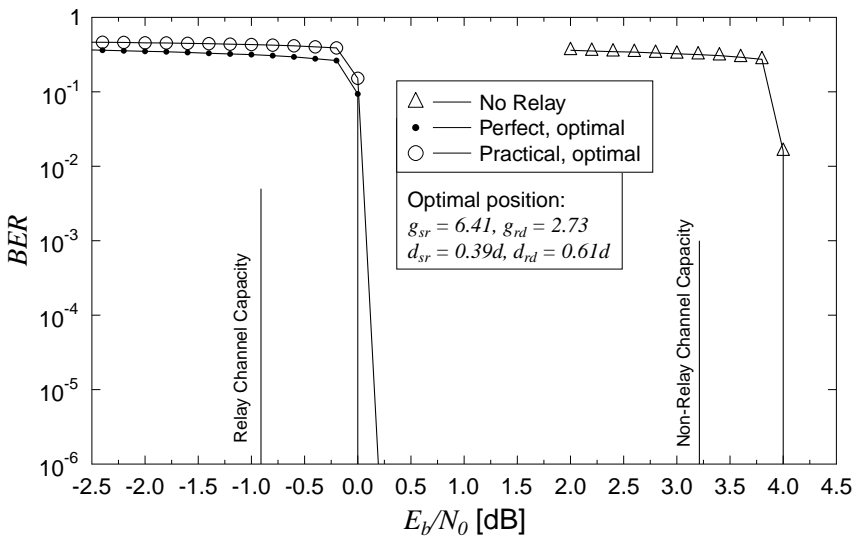

Fig. 29. BER-performance of the distributed coding aided system pictured in Fig. 25, when selecting $R$ nodes nearby the optimal position determined by Eq. (18). The scenarios considered corresponds to the configuration 3 and 4 mentioned in Tab. XI, where the 8PSK modulation scheme is chosen for the transmission link spanning from the $S$ node to the $R$ nodes, provided that all the transmissions in the system are carried out over Rayleigh-distributed small-scale fading channels.

The relay channel's capacity detailed in [17], [42] also suggests that there should be a different optimal position, when the objective function is that of maximising the relay channel's capacity. However, satisfying the optimal condition of Eq. (18), which is found by the EXIT chart analysis, always provides the best possible performance for the coding scheme, which is hence also better than that of the position that was optimally chosen for maximising the relay channel's capacity. Explicitly, depending on the specific choice of the optimisation cost-function (CF), diverse optimum solutions may be found and the system designer has to opt for using that specific CF, which is the most pertinent one for a particular service.

It should be noted that since a near-capacity design is applied for the DC-IrCC-URC-STTC scheme, no further system performance improvement may be achieved upon utilising at the $D$ node the signal transmitted from the $S$ node.

\section{Conclusions, Design guidelines And Future RESEARCH}

\section{A. Summary and Conclusions}

We have summarised the relevant EXIT-chart based design guidelines, which have been illustrated with the aid of various examples for diverse near-capacity wireless transceivers covering both the family of coherent and non-coherent based detection systems, MIMO systems and VMIMO systems. The IrCCURC-MPSK, IrCC-URC-DMPSK, IrCC-URC-STTC-MPSK and distributed IrCC-URC-STTC-MPSK coding schemes of Section V-B, Section V-C and Section VI have been respectively used as illustrative examples for demonstrating the application of the EXIT chart design tool in facilitating nearcapacity performance. In order to benchmark the near-capacity code design advocated, both the outage capacity and the OP of the schemes operating at the CCMC, at the DCMC as well as at the D-DCMC limits were presented. Specifically, the OP of the idealised at the DCMC and D-DCMC can be calculated by employing EXIT charts for determining the associated $S N R_{r}$ value corresponding to a given transmission rate $R$ of the systems of interest.

Additional discussions on the issue as to whether the coherent or the non-coherent scheme should be employed have been presented by providing a comparison of non-coherent versus coherent systems, where the effects of channel estimation errors were also alluded to. Moreover, for the sake of clarifying the trade-off between the system's latency and performance, the employment of sub-frame based transmissions was discussed, which has indicated the substantial benefit of employing the sub-frame transmission regime of Section V-D, when transmitting over slow fading channels.

The benefits of the EXIT chart-aided design principles in distributed coding schemes have been illustrated with the aid of the DC-IrCC-URC-STTC coding scheme, in the context of a single-user single-antenna based cooperative relaying system. In Section VI-D, we also presented a further application of EXIT charts in forming an optimisation criterion for selecting the relays by maintaining an open EXIT chart tunnel for all the composite coding arrangements of the distributed coding scheme.

\section{B. Design Guidelines}

In summary, the design process of the Irregular Concatenated Coding Arrangements (ICCAs) may be conducted in the following phases.

Phase 1 represents the specification of the basic design requirements, which includes the choices of the particular FEC scheme, the maximum code rate of the FEC scheme, the available modulation modes and their throughput, the tolerable complexity and latency, etc.

In phase 2, we need to quantify the capacity of the schemes stipulated in Phase 1, which are the outage capacity and the OP of the CCMC, of the DCMC as well as those of the D-DCMC. The OP of the DCMC and D-DCMC may be calculated based on the associated $S N R_{r}$ value corresponding to a given transmission rate $R$ and modulation scheme. Accordingly, the $S N R_{r}$ value may be determined by looking up the corresponding value on the capacity curves or by employing EXIT charts. The employment of EXIT charts used for formulating the capacity curve may be described in the following steps:

- EXIT curves pertaining to a given modulation scheme are generated for different $S N R_{r}$ values.

- A single point on the capacity curves is determined by computing the area under the EXIT curve and its corresponding $S N R_{r}$.

Phase 3 is dedicated for conducting the near-capacity design according to the scenario provided by the design requirement. The typical three component concatenated coding arrangement relying on IrCCs may be designed by carrying out the following steps:

- Optimise the inner-most two-stage coding arrangement by finding the most appropriate number of iterations associated with the affordable complexity.

- Create the EXIT curve of the amalgamated optimised inner decoder for different $S N R_{r}$ values. 
- Use the coding rate of the IrCC encoder specified by the design requirement and then employ the EXIT curve matching algorithm for generating the optimised weighting coefficients $\alpha_{i}$, where the number of IrCC subcodes is stipulated by the tolerable complexity of the design requirement.

\section{Future Research}

Future research may consider applying both the design principles as well as the resultant schemes in the context of cooperative communications. More particularly, it was suggested [163]-[165] that equalisers, namely Least Mean Square (LMS) equalisers or trellis based equalisers, can be incorporated into a cooperative communication system to exploit the joint advantages of having both relays and equalisers in dispersive scenarios. Once appropriate equalisers have been found for cooperative communication systems, we can integrate them with our existing coding schemes designed in Section V. Furthermore, we may employ these solutions in next-generation multi-user systems. Additionally, building upon the initial research on combining channel coding and network coding [166]-[168], cognitive radio schemes [169], [170] may be integrated into the system of [168] at the link level, where the knowledge of channel state information may be utilised for introducing adaptive channel coding and modulation into our system.

\section{REFERENCES}

[1] C. E. Shannon, "A mathematical theory of communication," Bell System Technical Journal, vol. 27, pp. 379-423 and 623-656, June and Oct. 1948.

[2] C. Berrou, A. Glavieux, and P. Thitimajshima, "Near shannon limit error-correcting coding and decoding: Turbo-codes. 1," in IEEE International Conference on Communications, 1993. ICC '93 Geneva. Technical Program, Conference Record, vol. 2, pp. 1064 -1070 vol.2, May 1993.

[3] C. Berrou and A. Glavieux, "Near optimum error correcting coding and decoding: Turbo codes," IEEE Transactions on Communications, vol. 44, pp. 1261-1271, Oct. 1996.

[4] C. Forney, "Concatenated codes," Cambridge: MIT Press, 1966.

[5] S. L. Goff, A. Glavieux, and C. Berrou, "Turbo-codes and high spectral efficiency modulation," in Proceedings of IEEE International Conference on Communications, pp. 645-649, 1994.

[6] S. Benedetto, D. Divsalar, G. Montorsi, and F. Pollara, "Bandwidth efficient parallel concatenated coding schemes," IEE Electronics Letters, vol. 31, pp. 2067-2069, 23rd Nov. 1995.

[7] P. Robertson and T. Worz, "Bandwidth-Efficient Turbo Trellis-Coded Modulation Using Punctured Component Codes," IEEE Journal on Selected Areas in Communications, vol. 16, pp. 206-218, Feb 1998.

[8] S. Benedetto, D. Divsalar, G. Montorsi, and F. Pollara, "Serial concatenation of interleaved codes: Performance analysis, design, and iterative decoding," IEEE Transactions on Information Theory, vol. 44, pp. 909 926, May 1998.

[9] L. Hanzo, T.H. Liew and B.L. Yeap, Turbo Coding, Turbo Equalisation and Space Time Coding for Transmission over Wireless channels. New York, USA: John Willy IEEE Press, 2002.

[10] J. H. Winters, J. Salz, and R. D. Gitlin, "The impact of antenna diversity on the capacity of wireless communication systems," IEEE Transactions on Communications, vol. 42, no. 234, pp. 1740-1751, 1994.

[11] V. Tarokh, N. Seshadri, and A. R. Calderbank, "Space-time codes for high data rate wireless communication: Performance criterion and code construction," IEEE Transactions on Information Theory, vol. 44, pp. 744-765, Mar. 1998

[12] S. M. Alamouti, "A simple transmit diversity technique for wireless communications," IEEE Journal on Selected Areas in Communications, vol. 16, pp. 1451-1458, Oct. 1998.
[13] V. Tarokh, A. Naguib, N. Seshadri, and A. R. Calderbank, "Space-time codes for high data rate wireless communication: Performance criteria in the presence of channel estimation errors, mobility, and multiple paths," IEEE Transactions on Communications, vol. 47, no. 2, pp. 199207, 1999.

[14] M. C. Valenti and B. Zhao, "Distributed turbo codes: towards the capacity of the relay channel," in IEEE 58th Proc. VTC 2003-Fall Vehicular Technology Conference 2003, vol. 1, pp. 322-326, Oct. 6-9, 2003.

[15] A. Sendonaris, E. Erkip, and B. Aazhang, "User cooperation diversity. Part I. System description," IEEE Transactions on Communications, vol. 51, pp. 1927-1938, Nov. 2003.

[16] J. N. Laneman, D. N. C. Tse, and G. W. Wornell, "Cooperative diversity in wireless networks: Efficient protocols and outage behavior," IEEE Transactions on Information Theory, vol. 50, pp. 3062-3080, Dec. 2004.

[17] A. Host-Madsen and J. Zhang, "Capacity bounds and power allocation for wireless relay channels," IEEE Transactions on Information Theory, vol. 51, pp. 2020-2040, June 2005.

[18] S. Yiu, R. Schober, and L. Lampe, "Distributed space-time block coding," IEEE Transactions on Communications, vol. 54, pp. 1195 1206, July 2006.

[19] P. Elias, "Coding for noisy channels," IRE Conv. Rec. pt.4, pp. 37-47, 1955.

[20] E. C. van der Meulen, "Three-terminal communication channels," Advanced Applied Probability, vol. 3, no. 1, pp. 120 - 154, 1971

[21] L. Bahl, C. Cullum, W. Frazer, and F. Jelinek, "An efficient algorithm for computing free distance," IEEE Transactions on Information Theory, vol. 18, pp. 437 - 439, May 1972.

[22] L. Bahl, J. Cocke, F. Jelinek, and J. Raviv, "Optimal decoding of linear codes for minimizing symbol error rate," IEEE Transactions on Information Theory, vol. 20, pp. 284 - 287, Mar. 1974.

[23] T. Cover and A. Gamal, "Capacity theorems for the relay channel," IEEE Transactions on Information Theory, vol. 25, pp. $572-584$, Sep. 1979.

[24] C. Douillard, A. Picart, M. Jézéquel, P. Didier, C. Berrou, and A. Glavieux, "Iterative correction of intersymbol interference: Turboequalization," European Transactions on Communications, vol. 6, pp. 507-511, 1995.

[25] V. Tarokh and N. Seshadri and A. Calderbank, "Space-time codes for high data rate wireless communications: Performance criterion and code construction," in Proc IEEE International Conference on Communications '97, (Montreal, Canada), pp. 299-303, 1997.

[26] S. Benedetto, D. Divsalar, G. Montorsi, and F. Pollara, "Serial concatenated trellis coded modulation with iterative decoding," in IEEE International Symposium on Information Theory Proceedings, pp. 8-, 1997.

[27] S. M. Alamouti, "A simple transmit diversity technique for wireless communications," IEEE Journal on Selected Areas in Coтmu., vol. 16, Oct. 1998.

[28] S. Benedetto, D. Divsalar, G. Montorsi, and F. Pollara, "Analysis, design, and iterative decoding of double serially concatenated codes with interleavers," IEEE Journal on Selected Areas in Communications, vol. 16, no. 2, pp. 231-244, 1998.

[29] S. ten Brink, "Convergence of iterative decoding," Electronics Letters, vol. 35, pp. 806-808, May 13, 1999.

[30] M.Tücher and J. Hagenauer, "Exit charts of irregular codes," in Proceeding of the 36th Annual Conference on Information Sciences and Systems [CDROM], (Princeton, NJ, USA), Mar. 2002.

[31] A. Ashikhmin, G. Kramer, and S. ten Brink, "Extrinsic information transfer functions: model and erasure channel properties," IEEE Transactions on Information Theory, vol. 50, pp. 2657-2673, Nov. 2004.

[32] S. ten Brink, "Convergence behavior of iteratively decoded parallel concatenated codes," IEEE Transactions on Communications, vol. 49 , pp. 1727-1737, Oct. 2001.

[33] M. F. U. Butt, S. X. Ng, and L. Hanzo, "Self-concatenated code design and its application in power-efficient cooperative communications," IEEE Communications Surveys Tutorials, vol. 14, no. 3, pp. 858-883, 2012.

[34] H. Chen, R. Maunder, and L. Hanzo, "A survey and tutorial on lowcomplexity turbo coding techniques and a holistic hybrid arq design example," IEEE Communications Surveys Tutorials, vol. PP, no. 99, pp. 1-21, 2013.

[35] P. Robertson and T. Worz, "Bandwidth-efficient turbo trellis-coded modulation using punctured component codes," IEEE Journal on Selected Areas in Communications, vol. 16, no. 2, pp. 206-218, 1998. 


\begin{tabular}{|c|c|}
\hline & LIST of ACRONYMS and SYMBOLS \\
\hline 16PSK & 16 level Phase Shift Keying \\
\hline 4PSK & 4-level Phase Shift Keying \\
\hline 8PSK & 8 level Phase Shift Keying \\
\hline BICM-ID & Bit-Interleaved Coded Modulation with Iterative Decoding \\
\hline BPS & Bit Per Symbol \\
\hline BPSK & Binary Phase-Shift Keying \\
\hline CCMC & Continuous-input Continuous-output Memoryless Channel \\
\hline CDD & Conventional Differential Detection \\
\hline CSI & Channel State Information \\
\hline DC-IrCC-URC-STTC & $\begin{array}{l}\text { Distributed Concatenated Irregular Convolutional Code, } \\
\text { Unity-Rate Code and Space-Time Trellis Code }\end{array}$ \\
\hline DCMC & Discrete-input Continuous-output Memoryless Channel \\
\hline D-DCMC & Differential Discrete-input Continuous-output Memoryless Channel \\
\hline DMPSK & Differential M-ary Phase Shift Keying \\
\hline EXIT & EXtrinsic Information Transfer \\
\hline FEC & Forward Error Control \\
\hline FER & Frame Error Ratio \\
\hline ICCAs & Irregular Concatenated Coding Arrangements \\
\hline IIR & Infinite Impulse Response \\
\hline $\mathrm{IrCCs}$ & Irregular Convolutional Codes \\
\hline IrCC-URC-DMPSK & $\begin{array}{l}\text { Irregular Convolutional Coded Unity Rate Coded } \\
\text { Differential M-ary Phase Shift Keying }\end{array}$ \\
\hline IrCC-URC-MPSK & $\begin{array}{l}\text { Irregular Convolutional Coded Unity Rate Coded } \\
\text { M-ary Phase Shift Keying }\end{array}$ \\
\hline IrCC-URC-STTC-MPSK & $\begin{array}{l}\text { Irregular Convolutional Coded Unity Rate Coded } \\
\text { Space Time Trellis Coded M-ary Phase Shift Keying }\end{array}$ \\
\hline LMS & Least Mean Square \\
\hline LTE & Long Term Evolution \\
\hline LLR & Loglikelihood Ratio \\
\hline MAP & Maximum A-Posteriori \\
\hline MIMO & Multiple Input Multiple Output \\
\hline ML & Maximum Likelihood \\
\hline MSDD & Multiple-Symbol Differential Detection \\
\hline MSDSD & Multiple-Symbol Differential Sphere Detection \\
\hline $\mathrm{OP}$ & Outage Probability \\
\hline $\mathrm{PCC}$ & Parallel Concatenated Convolutional \\
\hline PDF & Probability Density Function \\
\hline QPSK & Quadrature Phase-Shift Keying \\
\hline RSC & Recursive Systematic Convolutional \\
\hline $\mathrm{ScC}$ & Self-concatenated Convolutional \\
\hline SCC & Serial Concatenated Convolutional \\
\hline SISO & Single Input Single Output \\
\hline STBCs & Space Time Block Codes \\
\hline STTC & Space Time Trellis Coding \\
\hline STTCs & Space Time Trellis Codes \\
\hline TCM & Trellis Coded Modulation \\
\hline TCs & Turbo Codes \\
\hline URC & Unity Rate Codes \\
\hline$I_{A}$ & A priori mutual information \\
\hline$I_{A}^{i n}$ & $I_{A}$ at the inner component \\
\hline$I_{A}^{\text {out }}$ & $I_{A}$ at the outer component \\
\hline$I_{E}^{A}$ & Extrinsic mutual information \\
\hline$I_{E}^{i n}$ & $I_{E}$ at the inner component \\
\hline$I_{E}^{\text {out }}$ & $I_{E}$ at the outer component \\
\hline$L_{a}$ & A group of a priori LLRs \\
\hline$L_{e}$ & A group of extrinsic LLRs \\
\hline$\phi$ & Number of subcodes in IrCC \\
\hline$\alpha_{\phi}$ & Weighting coefficient of subcode $\phi$ \\
\hline$\beta_{\phi}$ & Coding rate of subcode $\phi$ \\
\hline$N_{t}$ & Number of transmit antenna \\
\hline$N_{w}$ & Window size \\
\hline$h_{s}$ & Slow fading coefficient \\
\hline$h_{f}$ & Fast fading coefficient \\
\hline$\eta$ & Modulated bits per symbol \\
\hline$R$ & Data transmission rate \\
\hline$R_{n}$ & Normalised throughput \\
\hline$\left.S N R_{r}\right|_{R}$ & Signal to noise ratio at the receiver, given $R$ \\
\hline$\left.S N R_{r}^{E X I T}\right|_{R}$ & $\left.S N R_{r}\right|_{R}$ calculated by the EXIT based method \\
\hline$\epsilon$ & Outage probability \\
\hline$I$ & Number of inner iterations \\
\hline$J$ & Number of outer iterations \\
\hline$T_{u}$ & SNR value corresponding to turbo cliff \\
\hline$D_{c}$ & Distance to the respective capacity \\
\hline$N_{s u b}$ & Number of sub-frames \\
\hline$\theta$ & Normalised channel estimation accuracy \\
\hline$d_{s r}$ & Distance form source to relay \\
\hline$g_{s r}$ & Power gain of the source-to-relay link \\
\hline$d_{r d}$ & Distance form relay to destination \\
\hline$g_{r d}$ & Power gain of the relay-to-destination link \\
\hline
\end{tabular}


[36] S. X. Ng, M. Butt, and L. Hanzo, "On the Union Bounds of SelfConcatenated Convolutional Codes," Signal Processing Letters, IEEE, vol. 16, pp. $754-757$, Sept. 2009.

[37] S. Howard and C. Schlegel, "Differential turbo-coded modulation with app channel estimation," IEEE Transactions on Communications, vol. 54, no. 8, pp. 1397-1406, 2006.

[38] R. Maunder, J. Wang, S. Ng, L. L. Yang, and L. Hanzo, "On the performance and complexity of irregular variable length codes for near-capacity joint source and channel coding," IEEE Transactions on Wireless Communications, vol. 7, no. 4, pp. 1338-1347, 2008.

[39] O. Alamri, J. Wang, S. X. Ng, L.-L. Yang, and L. Hanzo, “ NearCapacity Three-Stage Turbo Detection of Irregular Convolutional Coded Joint Sphere-Packing Modulation and Space-Time Coding ," IEEE Transactions on Communications, vol. 57, pp. 1486 -1495, May 2009.

[40] R. Maunder and L. Hanzo, "Near-capacity irregular variable length coding and irregular unity rate coding," IEEE Transactions on Wireless Communications, vol. 8, no. 11, pp. 5500-5507, 2009.

[41] R. Riaz, R. Maunder, M. Butt, S. Ng, S. Chen, and L. Hanzo, "Exit-chart-aided three-stage concatenated ultrawideband time-hopping spread-spectrum impulse radio design," IEEE Transactions on Vehicular Technology, vol. 58, no. 9, pp. 5320-5324, 2009.

[42] H. V. Nguyen, S. X. Ng, and L. Hanzo, "Distributed three-stage concatenated irregular convolutional, unity-rate and space-time trellis coding for single-antenna aided cooperative communications," in Vehicular Technology Conference Fall (VTC 2010-Fall), 2010 IEEE 72nd, pp. $1-5,2010$.

[43] R. Tee, R. Maunder, and L. Hanzo, "Exit-chart aided near-capacity irregular bit-interleaved coded modulation design," IEEE Transactions on Wireless Communications, vol. 8, no. 1, pp. 32-37, 2009.

[44] N. Wu and L. Hanzo, "Near-Capacity Irregular-Convolutional-CodingAided Irregular Precoded Linear Dispersion Codes," IEEE Transactions on Vehicular Technology, vol. 58, pp. 2863 -2871, July 2009.

[45] L. Kong, S. X. Ng, R. Maunder, and L. Hanzo, "Maximum-throughput irregular distributed space-time code for near-capacity cooperative communications," IEEE Transactions on Vehicular Technology, vol. 59, pp. 1511-1517, March 2010.

[46] L. Kong, S. X. Ng, R. Tee, R. Maunder, and L. Hanzo, "Reducedcomplexity near-capacity downlink iteratively decoded generalized multi-layer space-time coding using irregular convolutional codes,' IEEE Transactions on Wireless Communications, vol. 9, pp. 684-695, February 2010.

[47] R.-R. Chen, R. Peng, A. Ashikhmin, and B. Farhang-Boroujeny, "Approaching mimo capacity using bitwise markov chain monte carlo detection," IEEE Transactions on Communications, vol. 58, no. 2, pp. 423-428, 2010.

[48] N. Tran, T. Le-Ngoc, T. Matsumoto, and H. Nguyen, "Achieving nearcapacity performance on multiple-antenna channels with a simple concatenation scheme," IEEE Transactions on Communications, vol. 58, no. 4, pp. 1048-1059, 2010.

[49] L. Kong, S. X. Ng, R. Maunder, and L. Hanzo, "Near-capacity cooperative space-time coding employing irregular design and successive relaying," IEEE Transactions on Communications, vol. 58, pp. 2232 2241, August 2010.

[50] C. Xu, S. X. Ng, and L. Hanzo, "Near-capacity irregular convolutional coded cooperative differential linear dispersion codes using multiplesymbol differential detection," IEEE Signal Processing Letters, vol. 18, pp. 173-176, March 2011.

[51] L. Wang, L. Kong, S. X. Ng, and L. Hanzo, "Code-rate-optimized differentially modulated near-capacity cooperation," IEEE Transactions on Communications, vol. 59, no. 8, pp. 2185-2195, 2011.

[52] S. Sugiura, S. Chen, and L. Hanzo, "Mimo-aided near-capacity turbo transceivers: Taxonomy and performance versus complexity," IEEE Communications Surveys Tutorials, vol. 14, no. 2, pp. 421-442, 2012.

[53] S. Sugiura and L. Hanzo, "On the joint optimization of dispersion matrices and constellations for near-capacity irregular precoded spacetime shift keying," IEEE Transactions on Wireless Communications, vol. 12, no. 1, pp. 380-387, 2013.

[54] P. Zhang, S. Chen, and L. Hanzo, "Reduced-complexity near-capacity joint channel estimation and three-stage turbo detection for coherent space-time shift keying," IEEE Transactions on Communications, vol. 61, no. 5, pp. 1902-1913, 2013.

[55] R. G. Maunder, W. Zhang, T. Wang, and L. Hanzo, "A unary error correction code for the near-capacity joint source and channel coding of symbol values from an infinite set," IEEE Transactions on Communications, vol. 61, no. 5, pp. 1977-1987, 2013.
[56] M. El-Hajjar and L. Hanzo, "Exit charts for system design and analysis," IEEE Communications Surveys Tutorials, vol. PP, no. 99, pp. 1-27, 2013.

[57] Nasruminallah and L. Hanzo, "Near-capacity h.264 multimedia communications using iterative joint source-channel decoding," IEEE Communications Surveys Tutorials, vol. 14, no. 2, pp. 538-564, 2012.

[58] D. Divsalar, S. Dolinar, and F. Pollara, "Serial concatenated trellis coded modulation with rate-1 inner code," in IEEE Global Telecommunications Conference, 2000, vol. 2, pp. 777-782 vol.2, 2000.

[59] M. Tuchler, "Convergence prediction for iterative decoding of threefold concatenated systems," in Proc. IEEE Global Telecommunications Conference GLOBECOM 'O2, vol. 2, pp. 1358-1362, Nov. 17-21, 2002.

[60] S. X. Ng, J. Wang, M. Tao, L.-L. Yang, and L. Hanzo, "Iteratively decoded variable length space-time coded modulation: Code construction and convergence analysis," IEEE Transactions on Wireless Communications, vol. 6, pp. 1953-1963, May 2007.

[61] R. Tee, R. G. Maunder, and L. Hanzo, "Exit-chart aided near-capacity irregular bit-interleaved coded modulation design," IEEE Transactions on Wireless Communications, vol. 8, pp. 32-37, Jan. 2009.

[62] T. Richardson and R. Urbanke, "The capacity of low-density paritycheck codes under message-passing decoding," IEEE Transactions on Information Theory, vol. 47, pp. 599-618, Feb. 2001.

[63] T. Richardson, M. Shokrollahi, and R. Urbanke, "Design of capacityapproaching irregular low-density parity-check codes," IEEE Transactions on Information Theory, vol. 47, pp. 619-637, Feb. 2001.

[64] H. El-Gamal and J. Hammons, A.R., "Analyzing the turbo decoder using the gaussian approximation," IEEE Transactions on Information Theory, vol. 47, pp. 671-686, Feb. 2001.

[65] S. ten Brink, "Iterative decoding trajectories of parallel concatenated codes," in the 3rd IEEE/ITG Conference on Source and Channel Coding, pp. 75-80, Jan. 2000.

[66] S. ten Brink, "Design of serially concatenated codes based on iterative decoding convergence," in the 2nd International Symposium on Turbo Codes and Related Topics, pp. 319-322, Sept. 2000.

[67] H. Chen, R. Maunder, and L. Hanzo, "Low-complexity multiplecomponent turbo-decoding-aided hybrid arq," IEEE Transactions on Vehicular Technology, vol. 60, pp. 1571-1577, May 2011.

[68] J. Hagenauer, "The exit chart - introduction to extrinsic information transfer in iterative processing," in in Proceedings of the 12th European Signal Processing Conference (EUSIPCO), pp. 1541-1548, 2004.

[69] M. Tchler, S. T. Brink, and J. Hagenauer, "Measures for tracing convergence of iterative decoding algorithms," in in Proc. 4th IEEE/ITG Conf. on Source and Channel Coding, pp. 53-60, 2002.

[70] L. Hanzo, R. Maunder, J. Wang, and L. Yang, Near-Capacity VariableLength Coding:Regular and EXIT-Chart-Aided Irregular Designs. John Wiley \& Sons, 2011.

[71] A. Leon-Garcia, Probability and random processes for electrical engineering. Addison-Wesley, 2nd Edition, 1994.

[72] F. Brannstrom, L. Rasmussen, and A. Grant, "Convergence analysis and optimal scheduling for multiple concatenated codes," IEEE Transactions on Information Theory, vol. 51, pp. 3354-3364, Sept. 2005.

[73] W. N. Venables and B. D. Ripley, Modern applied statistics with SPLUS. New York: Springer-verlag, 1994

[74] S. ten Brink, "Convergence behavior of iteratively decoded parallel concatenated codes," IEEE Transactions on Communications, vol. 49, pp. 1727-1737, Oct. 2001.

[75] A. Ashikhmin, G. Kramer, and S. ten Brink, "Code rate and the area under extrinsic information transfer curves," in Proc. IEEE International Symposium on Information Theory, p. 115, 2002.

[76] S. X. Ng, S. Das, J. Wang, and L. Hanzo, "Near-Capacity Iteratively Decoded Space-Time Block Coding," in Proc. IEEE Vehicular Technology Conference VTC Spring 2008, pp. 590-594, May 11-14, 2008.

[77] S. ten Brink, "Rate one-half code for approaching the Shannon limit by 0.1 dB," Electronics Letters, vol. 36, pp. 1293-1294, July 20, 2000.

[78] M. Yang, W. Ryan, and Y. Li, "Design of efficiently encodable moderate-length high-rate irregular ldpc codes," IEEE Transactions on Communications, vol. 52, pp. 564-571, April 2004.

[79] T. Tian, C. Jones, J. Villasenor, and R. Wesel, "Selective avoidance of cycles in irregular ldpc code construction," IEEE Transactions on Communications, vol. 52, pp. 1242-1247, Aug 2004.

[80] H. Xiao and A. Banihashemi, "Improved progressive-edge-growth (peg) construction of irregular ldpc codes," IEEE Communications Letters, vol. 8, pp. 715-717, Dec 2004.

[81] A. Roumy, S. Guemghar, G. Caire, and S. Verdu, "Design methods for irregular repeat-accumulate codes," IEEE Transactions on Information Theory, vol. 50, pp. 1711-1727, Aug 2004. 
[82] G. Yue and X. Wang, "Optimization of irregular repeat accumulate codes for mimo systems with iterative receivers," IEEE Transactions on Wireless Communications, vol. 4, pp. 2843-2855, Nov 2005.

[83] I. Sason and R. Urbanke, "Complexity versus performance of capacityachieving irregular repeat-accumulate codes on the binary erasure channel," IEEE Transactions on Information Theory, vol. 50, pp. 1247 1256, June 2004

[84] G. Yue, X. Wang, and M. Madihian, "Design of rate-compatible irregular repeat accumulate codes," IEEE Transactions on Communications, vol. 55, pp. 1153-1163, June 2007.

[85] L. Hanzo, O.Alamri, M. E.Hajjar and N.Wu, Near-Capacity MultiFunctional MIMO Systems. New York, USA : John Wiley and Sons, 2009.

[86] D. Divsalar, S. Dolinar, and F. Pollara, "Iterative turbo decoder analysis based on density evolution," IEEE Journal on Selected Areas in Communications, vol. 19, pp. 891-907, May 2001.

[87] M. Tuchler, "Design of serially concatenated systems depending on the block length," IEEE Transactions on Communications, vol. 52, pp. 209-218, Feb. 2004

[88] F. Schreckenbach, N. Gortz, J. Hagenauer, and G. Bauch, "Optimization of symbol mappings for bit-interleaved coded modulation with iterative decoding," IEEE Communications Letters, vol. 7, pp. 593595, Dec. 2003.

[89] F. Simoens, H. Wymeersch, H. Bruneel, and M. Moeneclaey, "Multidimensional mapping for bit-interleaved coded modulation with BPSK/QPSK signaling," IEEE Communications Letters, vol. 9 , pp. 453-455, May 2005 .

[90] S. Pfletschinger and F. Sanzi, "Error floor removal for bit-interleaved coded modulation with iterative detection," IEEE Transactions on Wireless Communications, vol. 5, pp. 3174-3181, Nov. 2006.

[91] N. Muhammad and J. Speidel, "Joint optimization of signal constellation bit labeling for bit-interleaved coded modulation with iterative decoding," IEEE Communications Letters, vol. 9, pp. 775-777, Sept. 2005.

[92] F. Schreckenbach and G. B., "Bitinterleaved coded irregular modulation," European Transactions on Telecommunications, vol. 17, no. 2, pp. 269-282, 2006.

[93] S. ten Brink, G. Kramer, and A. Ashikhmin, "Design of low-density parity-check codes for modulation and detection," IEEE Transactions on Communications, vol. 52, pp. 670-678, May 2004.

[94] N. S. V. Tarokh, A. Naguib and A. Calderbank, "Space-time codes for high data rate wireless communications: Mismatch analysis," in Proc IEEE International Conference on Communications '97, (Montreal, Canada), pp. 309-313, 1997.

[95] A. F. Naguib, V. Tarokh, N. Seshadri, and A. R. Calderbank, "A spacetime coding modem for high-data-rate wireless communications," IEEE Journal on Selected Areas in Communications, vol. 16, pp. 1459-1478, Oct. 1998.

[96] L. Hanzo, S. X. Ng, W. Webb and T.Keller, Quadrature Amplitude Modulation: From Basics to Adaptive Trellis-Coded, Turbo-Equalised and Space-Time Coded OFDM, CDMA and MC-CDMA Systems, Second Edition. New York, USA : John Wiley and Sons, 2004.

[97] J. Lawton, "Theoretical error rates of differentially coherent binary and kineplex data transmission systems," Proceedings of the Institute of Radio Engineers, vol. 47, no. 2, pp. 333-334, 1959.

[98] J. Lawton, "Investigation of digital data communication systems," Cornell Aeronautical Lab., 1961.

[99] C. Cahn, "Performance of digital phase-modulation communication systems," IRE Transactions on Communications Systems, vol. 7, no. 1, pp. 3-6, 1959.

[100] P. Bello and B. D. Nelin, "The influence of fading spectrum on the binary error probabilites of incoherent and differentially coherent matched filter recievers," IRE Transactions on Communications Systems, vol. 10, pp. 160-168, June 1962.

[101] S. Wilson, J. Freebersyser, and C. Marshall, "Multi-symbol detection of M-DPSK," in IEEE Global Telecommunications Conference (GLOBECOM'89), pp. 1692-1697 vol.3, Nov. 1989.

[102] D. Divsalar and M. Simon, "Multiple-symbol differential detection of MPSK," IEEE Transactions on Communications, vol. 38, pp. 300-308, Mar. 1990.

[103] P. Ho and D. Fung, "Error performance of multiple-symbol differential detection of PSK signals transmitted over correlated Rayleigh fading channels," IEEE Transactions on Communications, vol. 40, pp. 1566 -1569 , Oct. 1992.

[104] D. Divsalar and M. Simon, "Maximum-likelihood differential detection of uncoded and trellis coded amplitude phase modulation over AWGN and fading channels-metrics and performance," IEEE Transactions on Communications, vol. 42, pp. 76-89, Jan. 1994.

[105] K. Mackenthun, "A fast algorithm for multiple-symbol differential detection of MPSK," IEEE Transactions on Communications, vol. 42, pp. 1471-1474, Feb. 1994.

[106] J. Cavers, "An analysis of pilot symbol assisted modulation for Rayleigh fading channels," IEEE Transactions on Vehicular Technology, vol. 40, pp. 686-693, Nov. 1991.

[107] H. Leib and S. Pasupathy, "The phase of a vector perturbed by Gaussian noise and differentially coherent receivers," IEEE Transactions on Information Theory, vol. 34, pp. 1491-1501, Nov. 1988.

[108] F. Edbauer, "Bit error rate of binary and quaternary DPSK signals with multiple differential feedback detection," IEEE Transactions on Communications, vol. 40, pp. 457-460, Mar. 1992.

[109] F. Adachi and M. Sawahashi, "Decision feedback multiple-symbol differential detection for M-ary DPSK," Electronics Letters, vol. 29, pp. 1385-1387, July 1993.

[110] P. Hoeher and J. Lodge, "'Turbo DPSK": iterative differential PSK demodulation and channel decoding," IEEE Transactions on Communications, vol. 47, pp. 837-843, June 1999.

[111] L. Lampe and R. Schober, "Iterative decision-feedback differential demodulation of bit-interleaved coded MDPSK for flat Rayleigh fading channels," IEEE Transactions on Communications, vol. 49, no. 7 , pp. 1176-1184, 2001.

[112] L. Lampe, R. Schober, V. Pauli, and C. Windpassinger, "Multiplesymbol differential sphere decoding," IEEE Transactions on Communications, vol. 53, pp. 1981 - 1985, Dec. 2005.

[113] V. Pauli, L. Lampe, and R. Schober, "'Turbo DPSK" using soft multiple-symbol differential sphere decoding," IEEE Transactions on Information Theory, vol. 52, pp. 1385 - 1398, April 2006.

[114] J. Jalden and B. Ottersten, "On the complexity of sphere decoding in digital communications," IEEE Transactions on Signal Processing, vol. 53, pp. 1474-1484, Apr. 2005.

[115] V. Pauli and L. Lampe, "On the complexity of sphere decoding for differential detection," IEEE Transactions on Information Theory, vol. 53, pp. 1595-1603, April 2007.

[116] D.Tse and P. Viswanath, Fundamentals of Wireless Communications. Englewood Cliffs, NJ, USA: Cambridge: Cambridge University Press, 2005.

[117] J. Laneman, D. Tse, and G. Wornell, "Cooperative diversity in wireless networks: Efficient protocols and outage behavior," IEEE Transactions on Information Theory, vol. 50, pp. 3062 - 3080, Dec. 2004.

[118] S. X. Ng and L. Hanzo, "On the mimo channel capacity of multidimensional signal sets," IEEE Transactions on Vehicular Technology, vol. 55, pp. 528 - 536, Mar. 2006.

[119] L. Li and A. Goldsmith, "Capacity and optimal resource allocation for fading broadcast channels .ii. outage capacity," Information Theory, IEEE Transactions on, vol. 47, pp. 1103-1127, Mar 2001.

[120] A. Avestimehr and D. Tse, "Outage capacity of the fading relay channel in the low-snr regime," Information Theory, IEEE Transactions on, vol. 53, pp. 1401-1415, April 2007.

[121] G. Levin and S. Loyka, "On the outage capacity distribution of correlated keyhole mimo channels," Information Theory, IEEE Transactions on, vol. 54, pp. 3232-3245, July 2008

[122] K. Kansanen and T. Matsumoto, "An analytical method for mmse mimo turbo equalizer exit chart computation," IEEE Transactions on Wireless Communications, vol. 6, pp. 59-63, Jan 2007.

[123] F. Babich, A. Crismani, and R. Maunder, "Exit chart aided design of periodically punctured turbo codes," Electronics Letters, vol. 46, pp. 1001-1003, July 2010.

[124] F. Babich and A. Crismani, "Cooperative coding schemes: Design and performance evaluation," IEEE Transactions on Wireless Communications, vol. 11, pp. 222-235, January 2012.

[125] X. Jaspar and L. Vandendorpe, "Joint source-channel codes based on irregular turbo codes and variable length codes," IEEE Transactions on Communications, vol. 56, pp. 1824-1835, November 2008.

[126] A. Elkhazin, K. Plataniotis, and S. Pasupathy, "Irregular convolutional codes in multiantenna bit-interleaved coded modulation under iterative detection and decoding," IEEE Transactions on Vehicular Technology, vol. 59, pp. 3332-3341, Sept 2010.

[127] A. Elnashar and M. El-Saidny, "Looking at lte in practice: A performance analysis of the lte system based on field test results," IEEE Vehicular Technology Magazine, vol. 8, pp. 81-92, Sept 2013.

[128] A. Larmo, M. Lindstrom, M. Meyer, G. Pelletier, J. Torsner, and H. Wiemann, "The lte link-layer design," IEEE Communications Magazine, vol. 47, pp. 52-59, April 2009. 
[129] D. Bai, C. Park, J. Lee, H. Nguyen, J. Singh, A. Gupta, Z. Pi, T. Kim C. Lim, M.-G. Kim, and I. Kang, "Lte-advanced modem design: challenges and perspectives," IEEE Communications Magazine, vol. 50, pp. 178-186, February 2012.

[130] Z. Hu, R. Susitaival, Z. Chen, I.-K. Fu, P. Dayal, and S. Baghel, "Interference avoidance for in-device coexistence in $3 \mathrm{gpp}$ lte-advanced: challenges and solutions," IEEE Communications Magazine, vol. 50, pp. 60-67, November 2012.

[131] A. Ghosh, R. Ratasuk, B. Mondal, N. Mangalvedhe, and T. Thomas, "LTE-advanced: next-generation wireless broadband technology [Invited Paper]," IEEE Wireless Communications, vol. 17, pp. $10-22$, June 2010.

[132] G. Piro, L. Grieco, G. Boggia, F. Capozzi, and P. Camarda, "Simulating LTE cellular systems: An open-source framework," IEEE Transactions on Vehicular Technology, vol. 60, pp. 498 -513, Feb. 2011.

[133] J. Yater, "Signal relay systems using large space arrays," IEEE Transactions on Communications, vol. 20, pp. 1108 - 1121, Dec. 1972.

[134] M. Hasna and M.-S. Alouini, "End-to-end performance of transmission systems with relays over rayleigh-fading channels," IEEE Transactions on Wireless Communications, vol. 2, pp. 1126 - 1131, Nov. 2003.

[135] L. Yang, M. Hasna, and M.-S. Alouini, "Average outage duration of multihop communication systems with regenerative relays," IEEE Transactions on Wireless Communications, vol. 4, pp. 1366 - 1371, July 2005

[136] M. Gastpar and M. Vetterli, "On the capacity of large gaussian relay networks," IEEE Transactions on Information Theory, vol. 51, pp. 765 - 779, Mar. 2005.

[137] Z. Zhang and T. Duman, "Capacity-approaching turbo coding and iterative decoding for relay channels," IEEE Transactions on Communications, vol. 53, pp. 1895 - 1905, Nov. 2005.

[138] A. Host-Madsen and J. Zhang, "Capacity bounds and power allocation for wireless relay channels," IEEE Transactions on Information Theory, vol. 51, pp. 2020 -2040, June 2005.

[139] W. Chin, Y. Qian, and G. Giambene, "Advances in cooperative and relay communications [guest editorial]," IEEE Communications Magazine, vol. 47, pp. 100 -101, Feb. 2009.

[140] K. Loa, C.-C. Wu, S.-T. Sheu, Y. Yuan, M. Chion, D. Huo, and L. Xu, "Imt-advanced relay standards [wimax/lte update]," IEEE Communications Magazine, vol. 48, pp. 40 -48, Aug. 2010.

[141] Y. Yu, R. Hu, C. Bontu, and Z. Cai, "Mobile association and load balancing in a cooperative relay cellular network," IEEE Communications Magazine, vol. 49, pp. 83 -89, May 2011.

[142] Y. Li, "Distributed coding for cooperative wireless networks: An overview and recent advances," IEEE Communications Magazine, vol. 47, pp. 71-77, August 2009.

[143] Z. Ding, K. Leung, D. Goeckel, and D. Towsley, "Opportunistic relaying for secrecy communications: Cooperative jamming vs. relay chatting," IEEE Transactions on Wireless Communications, vol. 10, pp. $1725-1729$, June 2011.

[144] L. Chen, R. Carrasco, and I. Wassell, "Opportunistic nonorthogonal amplify-and-forward cooperative communications," Electronics Letters, vol. 47, pp. $626-628$, Dec. 2011.

[145] M. Yassaee and M. Aref, "Slepian wolf coding over cooperative relay networks," IEEE Transactions on Information Theory, vol. 57, pp. 3462 -3482, June 2011.

[146] F. Tian, W. Zhang, W.-K. Ma, P. Ching, and H. Poor, "An effective distributed space-time code for two-path successive relay network," IEEE Transactions on Communications, vol. 59, pp. 2254 -2263, Aug. 2011

[147] Y. Chen, J. Zhang, and I. Marsic, "Link-layer-and-above diversity in multihop wireless networks," IEEE Communications Magazine, vol. 47, pp. $118-124$, Feb. 2009.

[148] A. Ibrahim, A. Sadek, W. Su, and K. Liu, "Cooperative communications with relay-selection: when to cooperate and whom to cooperate with?," Wireless Communications, IEEE Transactions on, vol. 7, pp. 2814-2827, July 2008.

[149] Y. Li and B. Vucetic, "On the performance of a simple adaptive relaying protocol for wireless relay networks," in Vehicular Technology Conference, 2008. VTC Spring 2008. IEEE, pp. 2400-2405, May 2008.

[150] Q. Xu, V. Stankovic, and Z. Xiong, "Distributed joint source-channel coding of video using raptor codes," IEEE Journal on Selected Areas in Communications, vol. 25, pp. 851-861, May 2007.

[151] I. Shahid and P. Yahampath, "Distributed joint source-channel coding using unequal error protection ldpc codes," IEEE Transactions on Communications, vol. 61, pp. 3472-3482, August 2013.
[152] A. Aljohani, S. X. Ng, and L. Hanzo, "Ttcm-aided rate-adaptive distributed source coding for rayleigh fading channels," IEEE Transactions on Vehicular Technology, vol. 63, pp. 1126-1134, March 2014.

[153] X. Bao and J. Li, "Adaptive network coded cooperation (ancc) for wireless relay networks: matching code-on-graph with network-on-graph," IEEE Transactions on Wireless Communications, vol. 7, pp. 574-583, February 2008

[154] L. Li, L. Wang, and L. Hanzo, "Generalized adaptive network coding aided successive relaying for noncoherent cooperation," IEEE Transactions on Communications, vol. 61, pp. 1750-1763, May 2013.

[155] X. Zhou, L.-L. Xie, and X. Shen, "Design of low-density paritycheck codes for half-duplex three-phase two-way relay channels," IEEE Transactions on Wireless Communications, vol. 13, pp. 1668-1677, March 2014

[156] J. Hu and T. Duman, "Low density parity check codes over wireless relay channels," IEEE Transactions on Wireless Communications, vol. 6 , pp. 3384-3394, September 2007.

[157] B. Zhao and M. Valenti, "Distributed turbo coded diversity for relay channel," Electronics Letters, vol. 39, pp. 786-787, May 2003.

[158] B. Zhang, H. Chen, M. El-Hajjar, R. Maunder, and L. Hanzo, "Distributed multiple-component turbo codes for cooperative hybrid arq," IEEE Signal Processing Letters, vol. 20, pp. 599-602, June 2013.

[159] S. Ng, Y. Li, B. Vucetic, and L. Hanzo, "Distributed irregular codes relying on decode-and-forward relays as code-components," IEEE Transactions on Vehicular Technology, vol. PP, no. 99, pp. 1-1, 2014.

[160] J. Yuan, Z. Chen, Y. Li, and L. Chu, "Distributed space-time trellis codes for a cooperative system," IEEE Transactions on Wireless Communications, vol. 8, pp. 4897-4905, October 2009.

[161] L. Kong, S. X. Ng, R. G. Maunder, and L. Hanzo, "Successive Relaying Aided Near-Capacity Irregular Distributed Space-Time Coding," in IEEE Global Telecommunications Conference, 2009. GLOBECOM 2009, pp. 1 -5, Nov. 30-Dec. 4, 2009.

[162] H. Ochiai, P. Mitran, and V. Tarokh, "Design and analysis of collaborative diversity protocols for wireless sensor networks," in Proc. VTC2004-Fall Vehicular Technology Conference 2004 IEEE 60th, vol. 7, pp. 4645-4649, Sept. 26-29, 2004.

[163] Y. Gong, Z. Ding, T. Ratnarajah, and C. Cowan, "Turbo channel estimation and equalisation for a superposition-based cooperative system," Communications, IET, vol. 3, pp. 1790-1799, November 2009.

[164] J. Zhang, L.-L. Yang, and L. Hanzo, "Energy-efficient dynamic resource allocation for opportunistic-relaying-assisted sc-fdma using turbo-equalizer-aided soft decode-and-forward," Vehicular Technology, IEEE Transactions on, vol. 62, pp. 235-246, Jan 2013.

[165] J. Li, D.-Z. Feng, and W. X. Zheng, "Space-time semi-blind equalizer for dispersive qam mimo system based on modified newton method," IEEE Transactions on Wireless Communications, vol. 13, pp. 3244 3256, June 2014.

[166] H. V. Nguyen, S. X. Ng, and L. Hanzo, "Performance bounds of network coding aided cooperative multiuser systems," IEEE Signal Processing Letters, vol. 18, no. 7, pp. 435-438, 2011.

[167] H. V. Nguyen, C. Xu, S. X. Ng, and L. Hanzo, "Non-coherent nearcapacity network coding for cooperative multi-user communications," IEEE Transactions on Communications, vol. 60, no. 10, pp. 3059-3070, 2012 .

[168] H. V. Nguyen, S. X. Ng, and L. Hanzo, "Irregular convolution and unity-rate coded network-coding for cooperative multi-user communications," IEEE Transactions on Wireless Communications, vol. 12 no. 3, pp. 1231-1243, 2013.

[169] D. Nguyen and M. Krunz, "Price-based joint beamforming and spectrum management in multi-antenna cognitive radio networks," IEEE Journal on Selected Areas in Communications, vol. 30, pp. 2295 2305, Dec. 2012.

[170] Q. Liang, S. Han, F. Yang, G. Sun, and X. Wang, "A distributedcentralized scheme for short- and long-term spectrum sharing with a random leader in cognitive radio networks," IEEE Journal on Selected Areas in Communications, vol. 30, pp. 2274 -2284, Dec. 2012. 


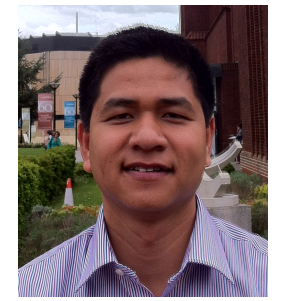

Hung Viet Nguyen received the B.Eng. degree in Electronics \& Telecommunications from Hanoi University of Science and Technology (HUST), Hanoi, Vietnam, in 1999, the M.Eng. in Telecommunications from Asian Institute of Technology (AIT), Bangkok, Thailand, in 2002 and the Ph.D. degree in wireless communications from the University of Southampton, Southampton, U.K., in 2013. Since 1999 he has been a lecturer at the Post \& Telecommunications Institute of Technology (PTIT), Vietnam. He is involved in the OPTIMIX and CONCERTO European projects. He is currently a postdoctoral researcher at Southampton Wireless (SW) group, University of Southampton, UK. His research interests include cooperative communications, channel coding, network coding and quantum communications.

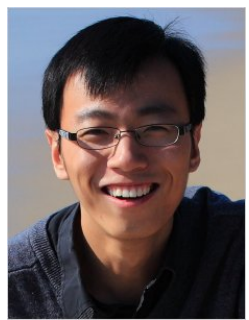

Chao Xu (S'09-M'14) received a B.Eng. degree from Beijing University of Posts and Telecommunications, Beijing, China, and a $\mathrm{BSc}($ Eng) with First Class Honours from Queen Mary, University of London, London, UK, through a Sino-UK joint degree program in 2008, both in Telecommunications Enginneering with Management. He obtained a MSc degree with distinction in Radio Frequency Communication Systems and a Ph.D. degree in Wireless Communications from the University of Southampton, Southampton, UK in 2009 and 2015, respectively. He is currently a postdoctoral researcher working at Southampton Wireless Group, University of Southampton, Southampton, UK. His research interests include reduced-complexity multiple-inputmultiple-output design, noncoherent detection, extrinsic-information-transfer-chart-aided turbo detection, and cooperative communications. He was awarded the Best M.Sc. Student in Broadband and Mobile Communication Networks by the IEEE Communications Society (United Kingdom and Republic of Ireland Chapter) in 2009, and he received 2012 Chinese Government Award for Outstanding Self-Financed Student Abroad.

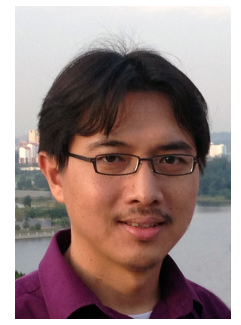

Dr Soon Xin Ng (S'99-M'03-SM'08) received the B.Eng. degree (First class) in electronic engineering and the Ph.D. degree in telecommunications from the University of Southampton, Southampton, U.K., in 1999 and 2002, respectively. From 2003 to 2006, he was a postdoctoral research fellow working on collaborative European research projects known as SCOUT, NEWCOM and PHOENIX. Since August 2006, he has been a member of academic staff in the School of Electronics and Computer Science, University of Southampton. He is involved in the OPTIMIX and CONCERTO European projects as well as the IU-ATC and UC4G projects. He is currently an Associate Professor in telecommunications at the University of Southampton.

His research interests include adaptive coded modulation, coded modulation, channel coding, space-time coding, joint source and channel coding, iterative detection, OFDM, MIMO, cooperative communications, distributed coding, quantum error correction codes and joint wireless-and-optical-fibre communications. He has published over 180 papers and co-authored two John Wiley/IEEE Press books in this field. He is a Senior Member of the IEEE, a Chartered Engineer and a Fellow of the Higher Education Academy in the UK.

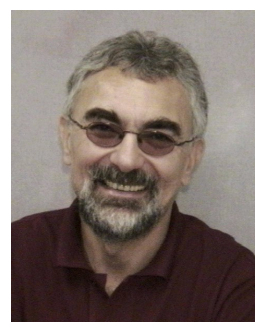

Lajos Hanzo (http://www-mobile.ecs.soton.ac.uk) FREng, FIEEE, FIET, Fellow of EURASIP, DSc received his degree in electronics in 1976 and his doctorate in 1983. In 2009 he was awarded an honorary doctorate by the Technical University of Budapest, while in 2015 by the University of Edinburgh. During his 38-year career in telecommunications he has held various research and academic posts in Hungary, Germany and the UK. Since 1986 he has been with the School of Electronics and Computer Science, University of Southampton, UK, where he holds the chair in telecommunications. He has successfully supervised about $100 \mathrm{PhD}$ students, co-authored 20 John Wiley/IEEE Press books on mobile radio communications totalling in excess of 10000 pages, published $1500+$ research entries at IEEE Xplore, acted both as TPC and General Chair of IEEE conferences, presented keynote lectures and has been awarded a number of distinctions. Currently he is directing a 60 -strong academic research team, working on a range of research projects in the field of wireless multimedia communications sponsored by industry, the Engineering and Physical Sciences Research Council (EPSRC) UK, the European Research Council's Advanced Fellow Grant and the Royal Society's Wolfson Research Merit Award. He is an enthusiastic supporter of industrial and academic liaison and he offers a range of industrial courses. He is also a Governor of the IEEE VTS. During 20082012 he was the Editor-in-Chief of the IEEE Press and a Chaired Professor also at Tsinghua University, Beijing. His research is funded by the European Research Council's Senior Research Fellow Grant. For further information on research in progress and associated publications please refer to http://wwwmobile.ecs.soton.ac.uk Lajos has $22000+$ citations. 